Review

\title{
Diversity of Pyrrolizidine Alkaloids in the Boraginaceae Structures, Distribution, and Biological Properties
}

\author{
Assem El-Shazly ${ }^{1}$ and Michael Wink ${ }^{2, *}$
}

1 Department of Pharmacognosy, Faculty of Pharmacy, Zagazig University, Zagazig 44519, Egypt;

E-Mail: assemels2002@yahoo.co.uk

2 Institute of Pharmacy and Molecular Biotechnology, Heidelberg University, Im Neuenheimer Feld 364, 69120 Heidelberg, Germany

* Author to whom correspondence should be addressed; E-Mail: Wink@uni-hd.de; Tel.: +49 6221-544881; Fax: 0049 6221-544884.

Received: 6 February 2014; in revised form: 24 February 2014 / Accepted: 13 March 2014 / Published: 1 April 2014

\begin{abstract}
Among the diversity of secondary metabolites which are produced by plants as means of defence against herbivores and microbes, pyrrolizidine alkaloids (PAs) are common in Boraginaceae, Asteraceae and some other plant families. Pyrrolizidine alkaloids are infamous as toxic compounds which can alkylate DNA und thus cause mutations and even cancer in herbivores and humans. Almost all genera of the family Boraginaceae synthesize and store this type of alkaloids. This review reports the available information on the present status (literature up to early 2014) of the pyrrolizidine alkaloids in the Boraginaceae and summarizes the topics structure, distribution, chemistry, chemotaxonomic significance, and biological properties.
\end{abstract}

Keywords: pyrrolizidine alkaloids; Boraginaceae; distribution; structures; NMR spectroscopy; mass spectrometry; biological properties

\section{Introduction}

Pyrrolizidine alkaloids (PAs) are common secondary metabolites in Boraginaceae, Fabaceae (tribe Crotalarieae) and Asteraceae (tribe Senecioneae) and serve as chemical defence compounds mainly against herbivores [1,2]. Several PA producing plants grow as weeds and therefore occur widely in agricultural production system throughout the world. They can enter the human food chain as a result 
of co-harvesting PA containing plants with edible grains. When bees visit areas, in which PA plants are abundant, PAs can be transferred into honey because nectar and pollen of PA plants contain alkaloids [3]. PA-contaminated human food can include cereals, milk, honey, eggs, and meat. In addition a PA-intake can occur when herbal teas and traditional medicines of the PA-containing plants are consumed [4-17].

Chronic health problems have been attributed to the presence of PAs in these products. The long-term toxicity is due to the conversion of pyrrolizidine alkaloids to the corresponding pyrrole derivatives which are highly reactive; they can alkylate DNA and have the ability to form DNA cross-linkage. As a consequence DNA replication is interrupted and mutations can occur, which can lead to liver and kidney cancer $[6,18,19]$. The ring nucleus (necine base) with a double bond in the 1:2 position is essential for genotoxic effects of theses alkaloids. PAs are bitter and modulate several neuroreceptors, including 5-HT receptors [20-22] which can induce immediate food avoidance in herbivores.

Many of the PAs have been shown to exhibit hepatotoxic, pulmotoxic, haemolytic, antimitotic, teratogenic, mutagenic and carcinogenic effects [22-36].

Plants of the family Boraginaceae are famous for the production of PAs. These alkaloids occur as free necines (either the necine base heliotridine or retronecine) or as a mixture of free bases and their $\mathrm{N}$-oxides. They can form single esters (monoester) at C-9 or C-7, open chain diesters at both C-7 and C-9 of the necine base, or in rare cases macrocyclic diesters linking C-7 with C-9 (Figures 1 and 2).

Several reviews on the occurrence of PAs have been published already [23-32,35,37-39] In the present review, we have made a complete review of PAs in Boraginaceae and have added also older records which were not covered so far. Tables 1 and 2 list the distribution of PAs in the Boraginaceae (a last comprehensive review was published by Hartmann and Witte [29]). GLC and GLC-MS data are tabulated in Table 3. For NMR data, we have only included NMR data of new PAs from the Boraginaceae in the time frame $1991-2013$ for ${ }^{13} \mathrm{C}$ (Table 4) and 1994-2013 for ${ }^{1} \mathrm{H}$ (Table 5).

Figure 1. Basic structures of pyrrolizidine alkaloids in Boraginaceae.

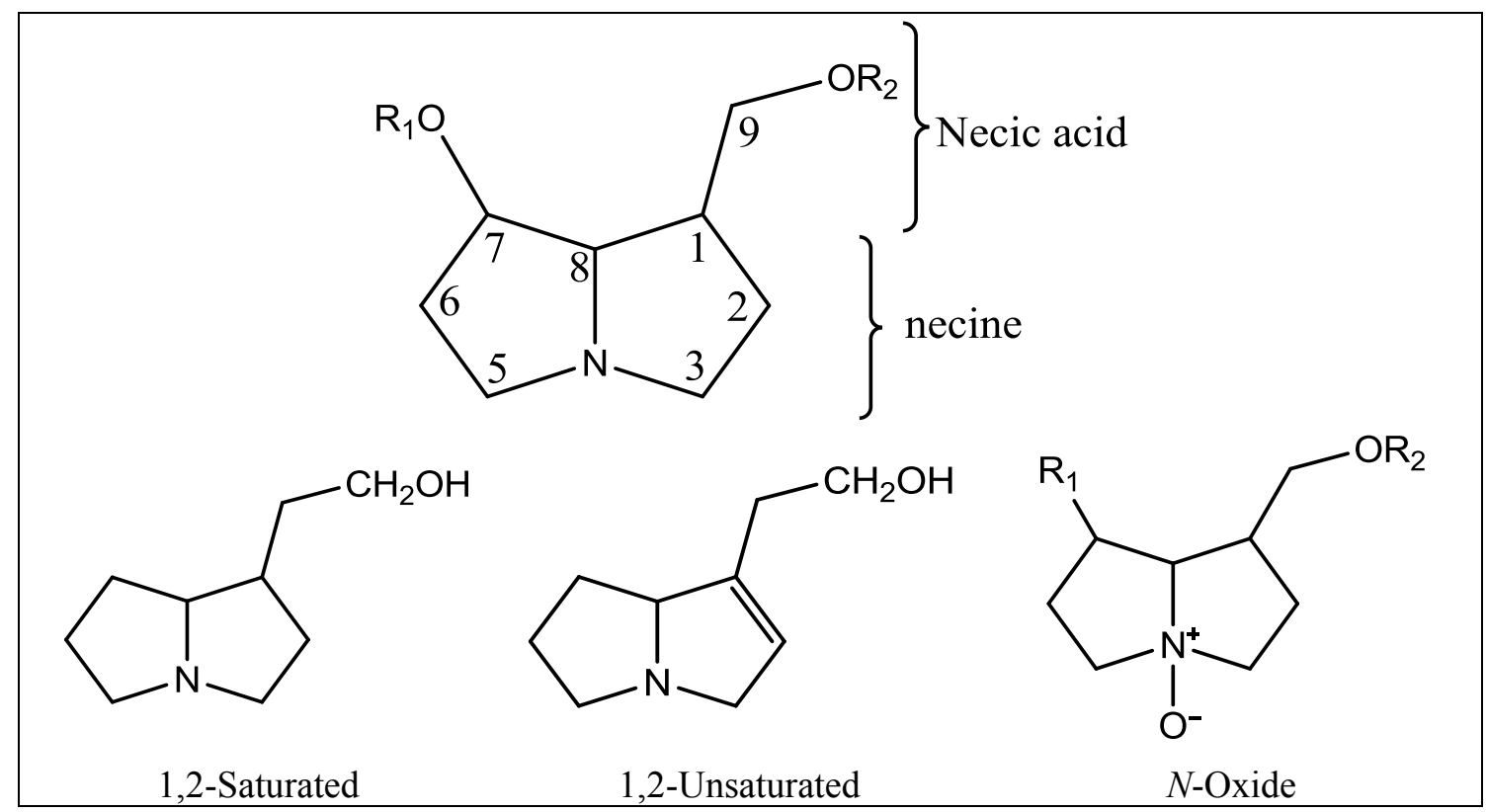


Figure 2. Necic acids occurring in pyrrolizidine alkaloids of Boraginaceae.

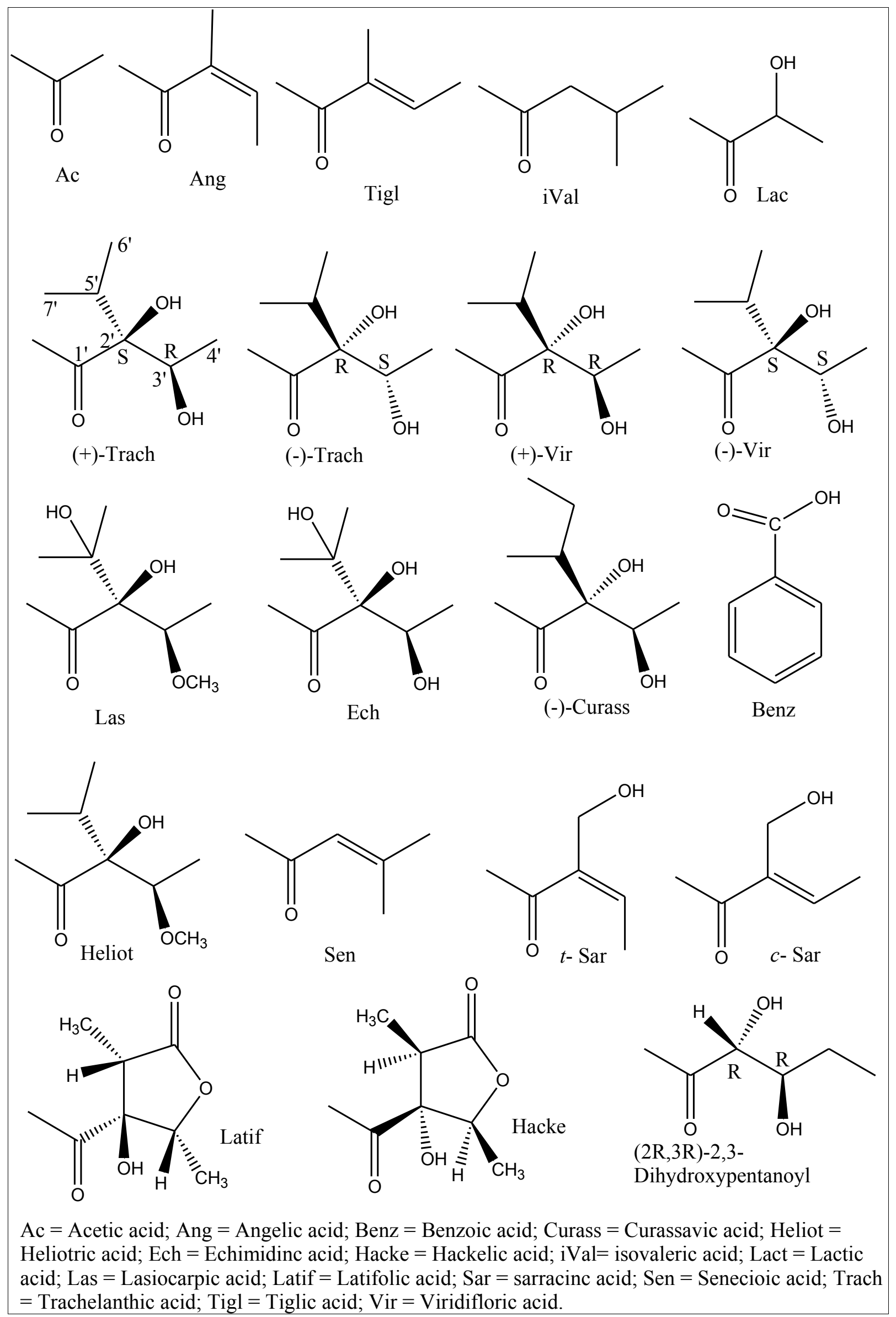


Table 1. List of plant species of the Boraginaceae containing PAs.

\begin{tabular}{|c|c|c|}
\hline Species & Pyrrolizidine alkaloids & References \\
\hline Alkanna orientalis & $\begin{array}{l}\text { 7-Angeloylretronecine, 9-angeloylretronecine, 7-tigloylretronecine, 9-tigloylretronecine, 7-seneioylretronecine, } \\
\text { 9-senecioylretronecine, triangularine, dihydroxytriangularine, triangularicine, dihydroxytriangularicine, } \\
\text { 7-angeloyl-9-(hydroxypropenoyl) retronecine, 7-tigloyl-9-(hydroxypropenoyl) retronecine, } \\
\text { 7-angeloyl-9-(2,3-dihydroxypropanoyl) retronecine, 7-tigloyl-9-(2,3-dihydroxypropanoyl) retronecine. }\end{array}$ & {$[40,41]$} \\
\hline $\begin{array}{l}\text { A. tinctoria } \\
\text { (A. tuberculata) }\end{array}$ & $\begin{array}{l}\text { 7-Angeloylretronecine, 7-tigloylretronecine, 9-tigloylretronecine, triangularine, dihydroxytriangularine, } \\
\text { triangularicine, dihydroxytriangularicine, 7-acetyl-9-sarracinoylretronecine, 7-angeloyl-9-(hydroxypropenoyl) } \\
\text { retronecine, 7-tigloyl-9-(hydroxypropenoyl) retronecine, 7-angeloyl-9-(2,3-dihydroxypropanoyl) retronecine, } \\
\text { 7-tigloyl-9-(2,3-dihydroxypropanoyl) retronecine. }\end{array}$ & {$[40,41]$} \\
\hline Amsinckia carinata & Echiumine, furcatine, 3'-acetylfurcatine, intermedine, 7 -acetylintermedine, lycopsamine, myoscorpine, supinine. & [42] \\
\hline A. douglasiana & Amabiline, cynaustrline, intermedine, lycopsamine, tessellatine, 9-(3'-acetylviridifloryl)turniforcidine. & {$[42,43]$} \\
\hline A. eastwoodiae & Amabiline, intermedine, 3 '-acetylintermedine, echiumine, lindelofine, lycopsamine, supinine, tessellatine. & [42] \\
\hline A. furacata & Furcatine, intermedine, 3'-acetylfurcatine, lycopsamine, supinine, tessellatine. & [42] \\
\hline A. grandiflora & Amabiline, intermedine, lycopsamine, 3'-aetyllycopsamine, 7-acetyllycopsamine, tessellatine, 9-acetyltessellatine. & [42] \\
\hline A. hispida & Echiumine, intermedine, lycopsamine. & [44] \\
\hline A. intermedia & Echiumine, intermedine, lycopsamine, sincamidine. & [44] \\
\hline A. lunaris & Lycopsamine, 3'-acetyllycopamine, intermedine, supinine, tesslatine, 9'-acetyltessellatine. & [42] \\
\hline A. lycopsoides & Amabiline, echiumine, intermedine, 3'-acetylintermedine, lycopsamine, supinine, tessellatine. & {$[42,44]$} \\
\hline $\begin{array}{l}\text { A. lycopsoides } \times \text { menziesii } \\
\text { var. intermedia }\end{array}$ & Amabiline, intermedine, 3'-acetylintermedine, lycopsamine, supinine, tessellatine. & {$[42]$} \\
\hline A. menziesii & Intermedine, lycopsamine, 3'-acetyllycopsamine, 7-acetyllycopsamine, 3',7-diacetyllycopsamine. & [45] \\
\hline $\begin{array}{l}\text { A. menziesii var. } \\
\text { intermedia }\end{array}$ & $\begin{array}{l}\text { Amabiline, cynaustraine (or steroisomer), echiumine, 3'-acetylechiumine, intermedine, 3'-acetylintermedine, } \\
\text { 7-acetylintermedine, 3',7-diacetylintermedine, lindelfoline, lycopsamine, 3'-acetyllycopsamine, } \\
\text { 7-acetyllycopsamine, myscorpine, 3'-acetylmyscorpine, supinine, symlandine, tessellatine. }\end{array}$ & {$[42,43]$} \\
\hline A. retrosa & Amabiline, intermedine, 3'-acetylintermedine, lycopsamine, myscorpine, 3'-acetylmyscorpine, supinine, tessellatine. & [42] \\
\hline $\begin{array}{l}\text { A. spectabilis var. } \\
\text { microcarpa }\end{array}$ & Intermedine, 3'-acetylintermedine, lindelofine, lycopsamine, myscorpine, supinine, tessellatine. & [42] \\
\hline $\begin{array}{l}\text { A. specabtilis var. } \\
\text { spectabilis }\end{array}$ & Intermedine, 3'-actylintermedine, lindelofine, lycopsamine, supinine. & [42] \\
\hline
\end{tabular}


Table 1. Cont

\begin{tabular}{|c|c|c|}
\hline Species & Pyrrolizidine alkaloids & References \\
\hline A. spectabilis var. nicolai & Intermedine, lindelofine, lycopsamine, tessellatine, trachelanthamine, supinine. & [42] \\
\hline A. tessellata & $\begin{array}{l}\text { Intermedine, lycopsamine, 3'-acetylintermedine, 3'-acetyllycopsamine, 7-acetylintermedine, 7-acetyllycopsamine, } \\
\text { 3',7-diacetylintermedine, 3'-7-diacetyllycopsamine. }\end{array}$ & [46] \\
\hline A. tessellata var. gloriosa & Amabiline, intermedine, lycopsamine, supinine, tessellatine, tracelanthamine, viridiflorine, $3^{\prime}$-acetylviridiflorine. & {$[42,43]$} \\
\hline A. tessellata var. tessellata & $\begin{array}{l}\text { Amabiline, intermedine, lycopsamine, 3'-acetyllycopsamine, tessellatine, 3'-acetyltessellatine, 9-acetyltessellatine, } \\
\text { 9,3'-acetyltessellatine, 9-(3'-acetylviridifloryl)-turniforcidine. }\end{array}$ & [42] \\
\hline A. vernicosa & Furcatine, intermedine, lycopsamine, supinine, 7-trachelanthyl retronecine. & [42] \\
\hline $\begin{array}{l}\text { Anchusa arvensis } \\
(=\text { Lycopsis arvensis) }\end{array}$ & $\begin{array}{l}\text { Echinatine, intermedine, 7-acetylintermedine, lycopsamine, 7-acetyllycopsamine, 3',7-diacetylintermedine (or its } \\
\text { isomer 3',7-diaetyllycopsamine), supinine, 9-acetyltrachelanthamidine, 9-angeloyltrachelanthamidine. }\end{array}$ & {$[40,47]$} \\
\hline $\begin{array}{l}\text { A. hispida } \\
(=\text { Gastrocotyle hispida })\end{array}$ & 7-Angeloylheliotridine, intermedine, lyopsamine, 7-acetyllycopsamine, retronecine, trachelanthamine and its isomer. & [40] \\
\hline A. milleri & $\begin{array}{l}\text { Heliotridine, 7-angeloylheliotridine, rinderine, retronecine, supinine, viridiflorine, 9-curassavoyleliotridine, } \\
\text { 7-acetyl-9-curassavoylheliotridine. }\end{array}$ & [40] \\
\hline A. officinalis & Intermedine, curassavine, lycopsamine, 7-aceyllycopsamine. & [47-49] \\
\hline A. strigosa & $\begin{array}{l}\text { Heliotridine 2S-hydroxy-2S(1S-hydroxyethyl)-4-methyl-pentanoyl ester, platyneine N-oxide 2S-hydroxy-2S(1S- } \\
\text { hydroxyethyl)-4-methyl-pentanoyl ester, retronecine 2S-hydroxy-2S(1S-hydroxyethyl)-4-methyl-pentanoyl ester and } \\
\text { its N-oxide, retronecine 2S-hydroxy-2S(1R-hydroxyethyl)-4-methyl-pentanoyl ester and its N-oxide, retronecine 2S- } \\
\text { hydroxy-2S(1S-hydroxyethyl)-[1'S-hydroxyethyl)-4-methylpentanoyl]-4-methyl-pentanoyl ester, supinidine N-oxide } \\
\text { 2S-hydroxy-2S(1S-hydroxyethyl)-4-methyl-pentanoyl ester, trachelanthamidine 2S-hydroxy-2S(1S-hydroxyethyl)-4- } \\
\text { methyl-pentanoyl ester. }\end{array}$ & {$[50,51]$} \\
\hline Arnebia decumbens & $\begin{array}{l}\text { 7-angeloylrtronecine, 9-angeloylretronecine, 7-tigloylrtronecine, 9-tigloylretronecine, europine, heliotrine, } \\
\text { lycopsamine, rinderine, supinine. }\end{array}$ & [52] \\
\hline Arnebia euchroma & 7-angeloylrtronecine, 9-angeloylretronecine & [53] \\
\hline A. hispidissima & Echimidine, monocrotaline. & [54] \\
\hline Asperugo procumbens & Amabiline (or supinine), echinatine. & [47] \\
\hline Borago officinalis & $\begin{array}{l}\text { Amabiline, intermedine, 7-acetylintermedine, lycopsamine, 7-acetyllycopsamine, supinine, thesinine, } \\
\text { thesinine-4'-O- } \beta \text {-D-glucoside. }\end{array}$ & {$[55,56]$} \\
\hline Caccinia crassifolia & Supinine, Heliotridine or retronecine trachelanthate. & [57] \\
\hline
\end{tabular}


Table 1. Cont

\begin{tabular}{|c|c|c|}
\hline Species & Pyrrolizidine alkaloids & References \\
\hline C. glauca & Retronecine 7:9 -dibenzoate. & [58] \\
\hline Cerinthe glabra & $\begin{array}{l}\text { Lycopsamine, 3'-acetyllycopsamine, 7-acetyllycopsamine, 3',7-diacetyllycopsamine, supinine, } \\
\text { 7-hydroxymethyl butyryl-9-viridifloryl-retronecine (or isomer). }\end{array}$ & [59] \\
\hline Cerinthe minor & Intermedine, 7-angeloylretronecine, 9-angeloyl-7-viridiflorylretronecine, lycopsamine. & {$[60,61]$} \\
\hline Cordia myxa & macrophylline & [62] \\
\hline C. sinensis & floridanine & [62] \\
\hline Cryptantha cana & $\begin{array}{l}\text { Intermdine, 3'-acetylintermedine, 7-acetylintemedine, lycopsamine, } \\
\text { 3'-acetyllycopsamine, 7-acetyllycopsamine. }\end{array}$ & [63] \\
\hline C. clevelandii & $\begin{array}{l}\text { Intermdine, 3'-acetylintermedine, echiumine, 2",3"-epoxyechiumine, thero-2',3' dihydroxyechiumine, } \\
\text { erytho-3"-chloro-2"-hydroxyechiumine. }\end{array}$ & [63] \\
\hline C. confertiflora & Amabiline, intermedine, lycopsamine, tessellatine, 9-acetyltessellatine. & [63] \\
\hline C. crassipes & $\begin{array}{l}\text { Lycopsamine, intermedine and their 3'-acetyllycopsamine, 3'-acetylintermedine, } 7 \text {-acetyllycopsamine, } \\
\text { 7-acetylintermedine, amabiline, echiumine, dihydroechumine, echimiplatine, lepanthine. }\end{array}$ & [64] \\
\hline C. fendleri & 7-Angeloylretronecine, 9-angeloylretronecine, latifoline, neolatifoline. & [63] \\
\hline C. flava & Intermedine, 3'-acetylintermedine, 7-acetylintemedine, lycopsamine, 3'-acetyllycopsamine, 7-acetyllycopsamine. & [63] \\
\hline C. inequata & Echimidine, acetylechimidine, echiuplatine, methylechiuplatine, lycopsamine, intermedine, dihydroxyechiumine. & [65] \\
\hline C. jamesii & Intermedine, lycopsamine, 3'- acetyllycopsamine, 7-aetyllyopsamine. & [66] \\
\hline C. leiocarpa & $\begin{array}{l}\text { 7-Angeloylretronecine, 9-angeloylretronecine, echiumine, intermedine, 3'-acetylintermedine, 2",3"-epoxyechiumine, } \\
\text { thero-2',3'-dihydroxyechiumine, erytho-3"-chloro-2"-hydroxyechiumine }\end{array}$ & [63] \\
\hline C. thyrsiflora & Intermedine, 3'-acetylintermedine, 7-acetylintemedine, lycopsamine, 3'-acetyllycopsamine, 7-acetyllycopsamine & [63] \\
\hline C. utahensis & Cryptanthine & [65] \\
\hline C. virgata & Intermedine, 3'-acetylintermedine, 7-acetylintemedine, lycopsamine, 3'-acetyllycopsamine, 7-acetyllycopsamine & [63] \\
\hline C. virginiensis & Amabiline, intermedine, lycopsamine, tessellatine. & [63] \\
\hline Cynoglossum amabile & $\begin{array}{l}\text { Amabiline, echinatine, 7-acetylechinatine, lycopsamine, rinderine, supinine. 9-angeloylretronecine, } \\
\text { 9-angeloyl-7-viridiflorylretronecine, }\end{array}$ & {$[60,67,68]$} \\
\hline C. australe & Cynausine, cynaustraline. & [68] \\
\hline C. clandestinum & 9-Angeloyl-7-viridiflorylretronecine, trachelanhamine & [60] \\
\hline C. columnae & N-oxides of echintine, rinderine, 3 '-acetylrinderine, 7-tigloyl-9-(2-deoxy-2-methyl)echimidinyl heliotridine. & [69] \\
\hline
\end{tabular}


Table 1. Cont.

\begin{tabular}{|c|c|c|}
\hline Species & Pyrrolizidine alkaloids & References \\
\hline C. creticum & $\begin{array}{l}\text { Cynoglossamine, echinatine, 3'-acetylechinatine, heliosupine, 3'-acetylheliosupine, 7-angeloylheliotridine, } \\
\text { 7-angeloyl-9-(methylbutyryl) heliotridine, } 7 \alpha \text {-angeloyl-1-chloromethyl-1,2-dehydropyrrolizidine, } \\
\text { 7-senecioylheliotridine, rinderine, 3'-acetylrenderine, supinine, trachelanthamine (or isomer). }\end{array}$ & [70-72] \\
\hline $\begin{array}{l}\text { C. furcatum } \\
\text { (C. zeylanicum) }\end{array}$ & Echinatine, isoechinatine, neocoromandaline, cynaustraline, lactodine, viridinatine & [73-76] \\
\hline C. germanicum & Echinatine, viridiflorine. & [77] \\
\hline C. glochidiatum & Amabiline & [78] \\
\hline C. lanceolatum & Cynaustine, cynaustraline. & [78] \\
\hline C. latifolium & 7-Angeloylheliotridine, latifoline. & [79] \\
\hline C. macrostylum & Echinatine, heliosupine. & [80] \\
\hline C. montanum & Cynaustine, cynaustraline, echinatine, heliosupine. & [81] \\
\hline C. nervosum & Heliotrine, echinatine, rinderine and their N-oxides. & [82] \\
\hline C. officinale & $\begin{array}{l}\text { Echinatine, 3'-acetylechinatine, 7-angeloylechinatine, heliosupine, 3'-acetylheliosupine, 7-angeloylheliotridine, } \\
\text { 7-angeloyl-1-formyl-6,7-dihydro-5H-pyrrolizidine, 7-angeloyl-9-(2-methylbutyryl)heliotridine, } \\
\text { 7-angeloyl-9-(2,3-dihydroxybutyryl) heliotridine, 7-tigloylheliotridine, rinderine, 7-angeloylrinderine, } \\
\text { trachelanthamine, viridiflorine. }\end{array}$ & {$[67,83,84]$} \\
\hline C. pictum & Echinatine, heliosupine, pictumine. & [85] \\
\hline C. viridiforum & Heliosupine, viridiflorine. & [86] \\
\hline Echium amoenum & Echimidine, echimidine isomer (tigloyl), 7-angeloylretronecine, 7-tigloylretronecine. & [87] \\
\hline E. angustifolium & Echimidine & [88] \\
\hline E. diffusum & Heliotridine or retronecine esters. & [28] \\
\hline E. glomeratum & 7-Angeloylretronecine, 9-angeloylretronecine, $(7 S, 8 R)$ petranine, $(7 S, 8 S)$ petranine. & [89] \\
\hline E. horridum & $\begin{array}{l}\text { Echimidine, echimidine isomer (tigloyl), lycopsamine, 7-acetyllycopsamine, 7-angeloyllycopsamine, } \\
\text { 7-tigloyllycopsamine, 7-angeloylretronecine, 7-tigloylretronecine, 7-angeloyl-9-(2-methylbutyryl)retronecine, } \\
\text { 7-tigloyl-9-(2-methylbutyryl) retronecine, 7-angeloyl-9-(2, 3-dihydroxybutyryl)retronecine, } \\
\text { 7-tigloyl-9-(2, 3-dihydroxybutyryl)retronecine, uplandicine. }\end{array}$ & [90] \\
\hline E. humile & $\begin{array}{l}\text { Echimidine, echihumiline, lycopsamine, 7-acetyllycopsamine, 7-senecioyllycopsamine, pycnanthine, } \\
\text { 7-seneioylretronecine, 9-seneioylretronecine, 7-(2-methylutyryl)retronecine, } \\
\text { 7-(methybutyryl)-9-(2,3-dihydroxybutyryl)retronecine, 7-(2-methybutyryl)-9-echimidinylretronecine. }\end{array}$ & [91] \\
\hline
\end{tabular}


Table 1. Cont.

\begin{tabular}{|c|c|c|}
\hline Species & Pyrrolizidine alkaloids & References \\
\hline E. hypertropicum & $\begin{array}{l}\text { Echimidine, echihumiline, 7-(2-methylbutyryl)-9-echimidinylretronecine, 7-senecioylretronecine, } \\
\text { 9-angeloylretronecine, lycopsamine, 7-acetyl-lycopsamine }\end{array}$ & [92] \\
\hline E. italicum & Echimidine & [28] \\
\hline $\begin{array}{l}\text { E. lycopsis } \\
(=\text { E. plantagineum })\end{array}$ & $\begin{array}{l}\text { Echimidine, echiumine, uplandicine, lycopsamine, intermedine, echimplatine, echiuplatine, 3'-acetylintermedine, } \\
\text { 3'-acetyllycopsamine, 3'-acetylechiumine, 9-angeloylretronecine, leptanthine. }\end{array}$ & {$[4,93,94]$} \\
\hline E. pininana & Echimidine, ehiupinine, 3'-aetylintermedine, hydroxymyoscorpine, myoscorpine. & [95] \\
\hline E. rauwolfii & $\begin{array}{l}\text { Echimidine, echimidine isomer (tigloyl), 7-acetyllycopsamine, 7-angeloyllycopsamine, 7-tigloyllycopsamine, } \\
\text { 7-angeloylretronecine, 7-tigloylretronecine, 7-angeloyl-9-(2-methylbutyryl)retronecine, } \\
\text { 7-tigloyl-9-(2-methylbutyryl)retronecine, 7-angeloyl-9-(2, 3-dihydroxybutyryl)retronecine, } \\
\text { 7-tigloyl-9-(2, 3-dihydroxybutyryl)retronecine, uplandicine. }\end{array}$ & [90] \\
\hline E. sericeum & Echimidine, symlandine (or symphytine). & [54] \\
\hline E. setosum & $\begin{array}{l}\text { Echimidine, echimidine isomer (tigloyl), 7-angeloylretronecine, 7-tigloylretronecine, 9-angeloylretronecine, } \\
\text { 9-tigloylretronecine, 7-angeloyl-9-(2-methylbutryl) retronecine, 7-tigloyl-9-(2-methylbutryl) retronecine, } \\
\text { 7-angeloyl-9-(2,3-dimethylbutryl) retronecine, 7-angeloyl-9-(2,3-dihydroxybutryl) retronecine, uplandicine. }\end{array}$ & [96] \\
\hline E. simplex & 7-Angeloylretronecine, 9-angeloylretronecine. & [61] \\
\hline $\begin{array}{l}\text { E. stenosiphon Webb } \\
\text { subsp. stenosiphon }\end{array}$ & Echimidine, 7-(2-methylbutyryl)-9-echimidinylretronecine & [92] \\
\hline E. tuberculatum & Echimidine,7-angeloyl-9-viridiflorylretronecine, 7-viridiflorylretronecine. & [60] \\
\hline E. vulgare & $\begin{array}{l}\text { Asperumine, heliosupine, 3'-acetylheliosupine, Echinatine, echiuplantine, leptanthine, echimiplantine, echivulgarine, } \\
\text { vulgarine, 7-O-acetylvulgarine, echimidine, echimidine isomer (tigloyl ?), 3'-acetylechimidine, 5'-acetylechimidine, } \\
\text { echihumiline, retronecine, 7-angeloylretronecine, 9-angeloylretronecine, 7-tigloylretronecine, 9-tigloylretronecine, } \\
\text { 7-(2-methylbutyryl) retronecine, 9-(2-methylbutyryl) retronecine, 7-angeloyl-9-(2-methylbutryl) retronecine, } \\
\text { 7-tigloyl-9-(2-methylbutryl) retronecine, 7-angeloyl-9-(2, 3-di methylbutryl) retronecine, } \\
\text { 7-tigloyl-9-(2, 3-dihydroxybutryl)retronecine, uplandicine }\end{array}$ & {$[11,96-98]$} \\
\hline E. wildpretti & Echimidine and its $\mathrm{N}$-oxide. & [99] \\
\hline Ehretia aspera & Ehretinine & [100] \\
\hline Eritrichium rupestre & 7-Angeloylretronecine, 7-angeloyl-9-viridiflorylretronecine, 7-viridiflorylretronecine & [60] \\
\hline Hackelia californica & Hackelidine, longitubine, 7-acetylhackelidine, 9-latifolylretronecine, 7-acetyl-9-latifolylretronecine & {$[101,102]$} \\
\hline
\end{tabular}


Table 1. Cont.

\begin{tabular}{|c|c|c|}
\hline Species & Pyrrolizidine alkaloids & References \\
\hline H. floribunda & Latifoline and its N-oxide. & [103] \\
\hline H. longituba & Latifoline, neolatifoline, longitubine, 7-angeloylretronecie, 9-angeloylretronecine. & [104] \\
\hline Heliotropium acutifolium & Heliotrine & [105] \\
\hline H. amplexicaule & Indicine & [29] \\
\hline H. angiospermum & Subulacine, lindelofidine, retronecine, supinidine, trachelanthamidine. & [106] \\
\hline H. arbainense & Europine, heliotrine, lasiocarpine. & [71] \\
\hline $\begin{array}{l}\text { H. arborescens } \\
(=\text { H. peruvianum) }\end{array}$ & Indicine, 3'-acetylindicine, lasiocarpine. & [107] \\
\hline H. arguzioides & Heliotrine, trichodesmine. & [28] \\
\hline H. bacciferum & Europine, heliotrine, heleurine and their N-oxides, supinine. & {$[108,109]$} \\
\hline H. bovei & Europine, 7-acetyleuropine, lasiocarpine, 5'-acetyllasiocarpine, lasiocarpine N-oxide, 5'-acetyllasiocarpine N-oxide. & [110] \\
\hline H. bracteatum & Helibractinecine, retronecine, helibracteatinine, helibracteatine & {$[111,112]$} \\
\hline H. bursiferum & 7-Angeloylretronecine. & [113] \\
\hline H. circinatum & 7-angeloylheliotrine, echinatine, europine, heleurine, heliotrine, lasiocarpine. & [114] \\
\hline H. confertifolium & lindelofidine, retronecine, supinidine, trachelanthamidine. & [106] \\
\hline H. crassifolium & Ilamine, europine and their N-oxides. & [115] \\
\hline H. curassavicum & $\begin{array}{l}\text { Coromandaline, coromandalinine, curassavine, curassavinine, curassanecine, heliocurassavine, heliocurassavinine, } \\
\text { heliocurassavicine, heliocoromandaline, heliovicine, } 7 \text {-angeloylheliotridine, trachelanhamidine, retronecine, supinidine. }\end{array}$ & {$[106,116-118]$} \\
\hline $\begin{array}{l}\text { H. curassavicum var. } \\
\text { argentium }\end{array}$ & 9-(3'-isovaleryl) viridifloryl retronecine, 9-(3'-acetyl) viridifloryl retronecine. & [119] \\
\hline $\begin{array}{l}\text { H. curassavicum var. } \\
\text { curassavicum }\end{array}$ & 9-(3'-isovaleryl) viridifloryl retronecine, 9-(3'-acetyl) viridifloryl retronecine. & [119] \\
\hline H. dasycarpum & heliotrine & [120] \\
\hline H. digynum (H. luteum) & Europine, heliotrine, 7-angeloylheliotrine, lasiocarpine. & [121] \\
\hline H. disciforme & Heliotrine, 2'-actylheliotrine, heliotrine $\mathrm{N}$-oxide, heleurine, heliorine $\mathrm{N}$-oxide. & [122] \\
\hline H. dissitiflorum & Heliotrine, heliotrine N-oxide, europine, 5'-deoxylasiocarpine. & [123] \\
\hline H. eichwaldii & Heliotrine, 7-angeloylheliotrine, lasiocarpine. & [124] \\
\hline H. esfandiarii & Europine, europine N-oxide. & [125] \\
\hline
\end{tabular}


Table 1. Cont

\begin{tabular}{|c|c|c|}
\hline Species & Pyrrolizidine alkaloids & References \\
\hline H. europaeum & $\begin{array}{l}\text { Europine, acetyleuropine, heleurine, heliotrine, } 7 \text {-angeloylheliotrine, lasiocarpine, 6-acetyllasiocarpine, heliotrine N- } \\
\text { oxide, dehydroheliotrine, 5'-acetyllasiocarpine N-oxide, N-(dihydropyrrolizinomethyl)-heliotrine, supinine. }\end{array}$ & {$[126,127]$} \\
\hline H. floridum & Floridine, floridinine, floridimine, heliovicine, 3 '-acetyltrachelanthamine. & [128] \\
\hline H. foliosisimum & lindelofidine, retronecine, supinidine, trachelanthamidine. & [106] \\
\hline H. fruticosum & lindelofidine, retronecine, supinidine, trachelanthamidine. & [106] \\
\hline H. hirsutissimum & $\begin{array}{l}\text { Europine, heliotrine, heleurine, lasiocarpine, 3'-acetyllasiocarpine, 5'-acetyllasiocarpine, supinine, } \\
\text { N-oxides of acetylasiocarpine, 3'-acetyleheliosupine. }\end{array}$ & {$[29,129]$} \\
\hline H. indicum & $\begin{array}{l}\text { Echinatine, helindicine, heliotrine, heleurine, indicine, acetylindicine, indicinine, lasiocarpine, lycopsamine, rinderine, } \\
\text { supinine, lindelofidine, retronecine, supinidine, trachelanthamine. }\end{array}$ & {$[106,116,130-132]$} \\
\hline H. keralense & Intermedine, isolycopsamine, retronesine. & [133] \\
\hline H. lasiocarpum & Heliotrine, lasiocarpine. & [29] \\
\hline H. marifolium & Europine, heliotrine, indicine, lasiocarpine. & [29] \\
\hline H. maris mortui & Europine, lasiocarpine. & {$[29,71]$} \\
\hline H. megalanthum & Lycopsamine, megalanthonine. & [134] \\
\hline H. molle & subulacine & [29] \\
\hline H. olgae & Heliotrine, incanine. & [135] \\
\hline H. ovalifolium & Heliofoline, retronecine. & [136] \\
\hline H. peruvianum & Rinderine & [29] \\
\hline H. popovii subsp. gillianum & Heliotrine & [28] \\
\hline H. procumbens & Lindelofidine, retronecine, supinidine, trachelanthamidine. & [106] \\
\hline H. queretaroanum & Lindelofidine, retronecine, supinidine, trachelanthamidine. & [106] \\
\hline H. racemosum & Lindelofidine, retronecine, supinidine, trachelanthamidine. & [106] \\
\hline H. ramosissimum & Heliotrine & {$[28]$} \\
\hline H. rotundifolium & Europine, 5'-acetyleuropine, heliotrine, lasiocarpine. & {$[137,138]$} \\
\hline H. scabrum & Heliscabine, retronecine. & [139] \\
\hline H. sessei & Lindelofidine, retronecine, supinidine, trachelanthamidine. & [106] \\
\hline H. spathulatum & $\begin{array}{l}\text { Amabiline, coromandaline, coromandalinine, heliovicine, curassavinine, curassavine, heliospathine, heliospathuline, } \\
\text { lindelofidine, retronecine, supinidine, trachelanthamidine. }\end{array}$ & {$[116,140]$} \\
\hline
\end{tabular}


Table 1. Cont.

\begin{tabular}{|c|c|c|}
\hline Species & Pyrrolizidine alkaloids & References \\
\hline H. steudneri & Lycopsamine & [27] \\
\hline H. strigosum & Strigosine, trachelanthamidine & {$[25,141]$} \\
\hline H. suaveolens & Echinatine, europine, heliotrine, lasiocarpine. & [29] \\
\hline H. subulatum & Subulacine; retronecine, heliotrine, 7 -angeloylheliotridine & [142] \\
\hline H. supinum & $\begin{array}{l}\text { Echinatine, heliosupine, heliotrine, 7-angeloylheliotridine (and its trachelanthic and viridifloric esters), lasiocarpine, } \\
\text { supinine. }\end{array}$ & {$[27,143]$} \\
\hline H. ternatum & subulacine & [27] \\
\hline H. transalpinum & Intermedine, indicine, lycopsamine, rinderine, 3'-acetylrinderine, supinine. & [144] \\
\hline $\begin{array}{l}\text { H. transalpinum var. } \\
\text { transalpinum }\end{array}$ & Transalpinecine, subulacine. & [145] \\
\hline H. transoxanum & Heliotrine & [105] \\
\hline H. wigginsii & Lindelofidine, retronecine, supinidine, trachelanthamidine. & [106] \\
\hline Lappula glochidiata & Echinatine & [146] \\
\hline L. intermedia & & [147] \\
\hline $\begin{array}{l}\text { (Echinospermum } \\
\text { intermedium) }\end{array}$ & Lasiocarpine & \\
\hline L. myosotis & Intermedine, lycopsamine, 7 -acetylintermedine, 7-acetyllycopsamine. & {$[35,148]$} \\
\hline L. spinocarpos & $\begin{array}{l}\text { Amabiline, intermedine, } 7 \text {-angeloylheiotridine, 9- heliotrinoylretronecine, lycopsamine, 7-acetyllycopsamine, } \\
\text { retronecine, trachelanthamine, supinine, viridiflorine. }\end{array}$ & [40] \\
\hline $\begin{array}{l}\text { Lindelofia anchusoides } \\
\text { (L. macrostyla) }\end{array}$ & Lindelofamine, lindelofine. & [28] \\
\hline L. angustifolia & Amabiline, echinatine & [78] \\
\hline L. longiflora & Echinatine and its N-oxide. & [149] \\
\hline L. olgae & Viridiflorine & {$[150]$} \\
\hline L. pterocarpa & Viridiflorine & [151] \\
\hline L. spectabilis & Echinatine, 3'-acetylechinatine, monocorotaline. & [124] \\
\hline L. stylosa & Echinatine, lindelofine, viridiflorine. & [135] \\
\hline L. tschimganica & Echinatine, carategine, viridiflorine. & [152] \\
\hline
\end{tabular}


Table 1. Cont.

\begin{tabular}{|c|c|c|}
\hline Species & Pyrrolizidine alkaloids & References \\
\hline Lithospermum canesens & $\begin{array}{l}\text { Canesine, canescenine, 3'-acetycanesine, 3'-acetylcanescenine, lycopsamine, 7-acetyllycopsamine, } \\
\text { 7-acetylintermedine. }\end{array}$ & {$[153,154]$} \\
\hline L. erythrorhizon & Intermedine, myoscorpine, hydroxymyoscorpine. & [155] \\
\hline L. officinale & Lithosenine, acetyllithosenine. & [156] \\
\hline L. purpureocoeruleum & Lycopsamine & {$[60]$} \\
\hline Macrotomia echioides & Macrotomine & [28] \\
\hline Mertensia bakeri & Lycopsamine & [157] \\
\hline M. ciliata & Intermedine, lycopsamine. & [157] \\
\hline Messerschmidia argentea & Indicine, $3^{\prime}$-acetylindicine, and their $\mathrm{N}$-oxide. & [158] \\
\hline M. sibirica & Lycopsamine, 9-angeloylretronecine. & {$[28]$} \\
\hline $\begin{array}{l}\text { Moltikiopsis ciliata } \\
\text { (Lithospermum callosum) }\end{array}$ & Echinatine, heliotrine. & [108] \\
\hline $\begin{array}{l}\text { Myosotis scorpioides } \\
(=\text { M. palustris) }\end{array}$ & Myoscorpine, symphytine, scorpioidine, 7-acetylscorpioidine. & [159] \\
\hline M. sylvatica & Heliosupine, 3'-acetylheliosupine, 9-angeloylretronecine, trachelanthamine. & {$[25]$} \\
\hline Neatostema apulum & Amabiline, lycopsamine and their N-oxides. & [160] \\
\hline Nonnea lutea & 7-viridiflorylretronecine & {$[60]$} \\
\hline N. setosa & 7-viridiflorylretronecine & [60] \\
\hline Omphalodes verna & Isoretronocanol or its isomer. & {$[27]$} \\
\hline Onosma alborosea & Intermedine, lycopsamine, 7-acetylintermedine, 7-acetylycopsamine. & [161] \\
\hline $\begin{array}{l}\text { O. alboroseum } \times \\
\text { sanguinolentum }\end{array}$ & 9-Angeloylretronecine, echimidine, lycopsamine, intermedine, 7-acetylintermedine, 7-acetylycopsamine. & {$[161,162]$} \\
\hline O. arenaria & $\begin{array}{l}\text { 7-Acetyllycopsamine, 5,6-diydro-7,9-dimethoxy-7H-pyrrolizine, 7-acetylretronecine, } \\
\text { 7-acetyl-9-(2-methylbutryl) retronecine, 7-acetyl-9-(2,3-dimethylbutryl) retronecine, } \\
\text { 7-acetyl-9-(2-hydroxy-3-methylbutryl) retronecine, 7-acetyl-9-(2,3-dihydroxybutryl) retronecine, } \\
\text { 9-(butyryl-2-ene) supinidine, 3'-acetylsupinine, uplandicine. }\end{array}$ & [163] \\
\hline $\begin{array}{l}\text { O. arenaria subsp. } \\
\text { pennina }\end{array}$ & Intermedine, lycopsamine, 7-acetylintermedine, 7-acetylycopsamine. & [161] \\
\hline
\end{tabular}


Table 1. Cont

\begin{tabular}{|c|c|c|}
\hline Species & Pyrrolizidine alkaloids & References \\
\hline O. erecta & N-oxides of 7-O-acetylechinatine, viridinatine, stereoisomer, 7-epi-echimiplatine, onosmerectine. & [164] \\
\hline O. hetrophyllum & Helioridine, 1-methylene- $8 \alpha$-pyrrolizidine. & [165] \\
\hline O. leptantha & Echihumiline, 3'-acetylechihumiline, leptanthine and their N-oxides. & [166] \\
\hline O. stellulatum & $\begin{array}{l}\text { Echimidine, 7-viridiflorylretronecine, heliospathuline, leptanthine, lyopsamine and heir N-oxides, } \\
\text { 7-acetylintermedine, dihydroechinatine, trahelanthamine, uplandicine. }\end{array}$ & {$[60,167]$} \\
\hline Paracaryum himalayense & Viridiflorine & [151] \\
\hline P. intermedium & 7-Angeloylheliotridine, 7-senecioylheliotridine, rinderine, 7-angeloylrindrine, 7 -senecioylrinderine, viridiflorine. & {$[40]$} \\
\hline P. regulosum & Echinatine, heliosupine, 7-angeloylheliotridine, rinderine, viridiflorine. & [40] \\
\hline $\begin{array}{l}\text { Paracynoglossum } \\
\text { imeretinum }\end{array}$ & Echinatine, heliosupine. & [29] \\
\hline Pulmonaria obscura & Intermedine, lycopsamine, 7-acetylintermedine, 7 -acetylycopsamine. & {$[168]$} \\
\hline Rindera austroechinata & Echinatine, rinderine, 7 -angeloylheliotridine & {$[57,152]$} \\
\hline R. baldschuanica & Echinatine, rinderine, trachelanthamine, turkestanine & {$[152]$} \\
\hline R. cyclodonta & Echinatine & [120] \\
\hline R. echinata & Echinatine, trachelanthamine & [120] \\
\hline R. oblongifolia & Cerategine, echinatine, turkestanine. & {$[152]$} \\
\hline R. umbellata & $\begin{array}{l}\text { 7-Angeloyl-9-(+)-trachelanthyl heliotridine, lindelofine, punctanecine, 7-angeloyl heliotridane, 7-angeloyl } \\
\text { heliotridine, heliosupine, 9-(+)-trachelanthyl-laburnine, echinatine. }\end{array}$ & [169] \\
\hline Solenanthus circinnatus & Echinatine & [151] \\
\hline S. coronatus & Echinatine & [135] \\
\hline S. karateginus & Cerategine, ehinatine. & [151] \\
\hline S. turkestanicus & Rinderine, turkestanine. & {$[135,150]$} \\
\hline Symphytum aintabicum & Echimidine & [170] \\
\hline S. asperum & $\begin{array}{l}\text { Echimidine, symphytine, asperumine, ehinatine, heliosupine, acetylechimidine (or its isomer), aetyllyopsamine (or its } \\
\text { isomer), symviridine }\end{array}$ & {$[171,172]$} \\
\hline S. bohemium & Echimidine, lycopsamine, 7-acetyllycopsamine, symphytine. & [173] \\
\hline S. caucasium & Asperumine, echimidine, echinatine, heliotrine, lasiocarpine. & [174] \\
\hline S. consolidum & Echimidine, symphytine. & [175] \\
\hline
\end{tabular}


Table 1. Cont.

\begin{tabular}{|c|c|c|}
\hline Species & Pyrrolizidine alkaloids & References \\
\hline S. grandiflorum & Echimidine, lycopsamine, symphytine. & [176] \\
\hline S. ibericum & Echimidine, lycopsamine, symphytine. & [176] \\
\hline S. officinale & $\begin{array}{l}\text { Lycopsamine, 7-acetyllycopsamine, symphytine, echimidine, echinatine, heliosupine, intermedine, } \\
\text { 7-acetylintermedine, viridiflorine, symviridine. }\end{array}$ & $\begin{array}{l}{[17,162,171,} \\
172,177-179]\end{array}$ \\
\hline S. orientale & Anadoline, echimidine, symphytine. & [28] \\
\hline S. peregrinum & Intermedine, 7-acetylintermedine, lycopsamine, 7-acetyllycopsamine, symphytine. & [27] \\
\hline $\begin{array}{l}\text { S. sylvaticum subsp. } \\
\text { sepulcrale var. sepulcrale }\end{array}$ & Echimidine N-oxide & [170] \\
\hline S. tanaiense & Echimidine, lycopsamine, 7-acetyllycopsamine, symphytine. & [173] \\
\hline S. tuberosum & 7-Angeloylretronecine; anadoline, echimidine, lycopsamine, 7-acetyllycopsamine, symphytine. & {$[28,162,176]$} \\
\hline$S \times$ uplandicum & $\begin{array}{l}\text { Echimidine, intermedine, } 7 \text {-acetylintermedine lycopsamine, } 7 \text {-acetyllycopsamine, symphytine, symlandine, } \\
\text { symviridine, uplandicine. }\end{array}$ & {$[172,180]$} \\
\hline Tournefortia sarmentosa & Supinine & [28] \\
\hline T. sibirica & Turneforcine & [29] \\
\hline T. sogdiana & Echinatine & [57] \\
\hline Trahlenthus hissaricus & Trachelanthine, trachelanthamine, viridiforine. & [29] \\
\hline T. korolkovii & Trachelanthine, trachelanthamidine, trachelanthamine. & {$[57,150]$} \\
\hline Trichodesma africanum & Europine, intermedine, lycopsamine, trichodesmine, retronecine, viridiflorine & {$[40,71,181]$} \\
\hline T. ehrenbergii & Senkirkine, supinine. & [54] \\
\hline T. incanum & Inanine, trihodesmine. & [46] \\
\hline T. zeylanicum & Supinine & [182] \\
\hline Ulugbekia tschimganica & Uluganine & [183] \\
\hline
\end{tabular}


Table 2. Alkaloid composition of investigated species of Boraginaceae.

\begin{tabular}{|c|c|c|}
\hline Compounds & Sources & References \\
\hline 3'-Acetylcanesine & Lithospermum canesens & {$[153,154]$} \\
\hline 3'-Acetylcanescenine & Lithospermum canesens & {$[153,154]$} \\
\hline 7-Acetyl-9-curassavoylheliotridine & Anchusa milleri & {$[40]$} \\
\hline 7-Acetyl-9-(2,3-dihydroxybutryl) retronecine & Onosma arenaria & [163] \\
\hline 7-Acetyl-9-(2-dimethylbutryl) retronecine & Onosma arenaria & [163] \\
\hline \multirow[t]{4}{*}{ 3'-Acetylechinatine } & Cynoglossum creticum & {$[70]$} \\
\hline & Cynoglossum officinale & [67] \\
\hline & Lindelofia spectabilis & {$[124]$} \\
\hline & Messerschmidia argentea & {$[158]$} \\
\hline \multirow[t]{3}{*}{ 7-Acetylechinatine } & Cynoglossum amabile & {$[67]$} \\
\hline & Cynoglossum officinale & {$[67,84]$} \\
\hline & Onosma erecta & {$[69]$} \\
\hline 3'- Acetylechihumiline & Onosma leptantha & [166] \\
\hline \multirow[t]{2}{*}{ 3'-Acetylechiumine } & Amsinckia menziessi var. intermedia & {$[42]$} \\
\hline & Cryptantha clevelandii & [63] \\
\hline 3'-Acetylechimidine & Echium vulgare & [96] \\
\hline 5'-Acetylechimidine & Echium vulgare & [11] \\
\hline \multirow[t]{2}{*}{ 5'-Acetyleuropine } & Heliotropium disciforme & [122] \\
\hline & Heliotropium rotundifloium & [138] \\
\hline 7-Acetyleuropine & Heliotropium bovi & {$[110]$} \\
\hline \multirow[t]{2}{*}{ 3'-Acetylfurcatine } & Amsinckia carinata & {$[42]$} \\
\hline & Amsinckia furacata & {$[42]$} \\
\hline \multirow[t]{3}{*}{ 3'-Acetylheliosupine } & Cynoglossum creticum & {$[70]$} \\
\hline & Cynoglossum officinale & {$[67,83]$} \\
\hline & Heliotropium hirsutissimum & {$[129]$} \\
\hline 7-Acetyl-9-(2-hydroxy-3-methylbutryl) retronecine & Onosma arenaria & [163] \\
\hline \multirow[t]{2}{*}{ 3'-Acetylindicine } & Heliotropium arborescens (H. peruvianum) & [28] \\
\hline & Messerschmidia argentea & [158] \\
\hline \multirow[t]{16}{*}{ 3'-Acetylintermedine } & Amsinckia eastwoodiae & {$[42]$} \\
\hline & Amsinckia lycopsoides & [42] \\
\hline & $\begin{array}{l}\text { Amsinckia lycopsoides } \times \text { menziesii } \\
\text { var. intermedia }\end{array}$ & [42] \\
\hline & Amsinckia menziesii var. intermedia & [43] \\
\hline & Amsinckia retrosa & [42] \\
\hline & Amsinckia spectabilis var. microcarpa & [42] \\
\hline & Amsinckia spectabilis var. spectabilis & [42] \\
\hline & Amsinckia tessellata & [46] \\
\hline & Cryptantha cana & [63] \\
\hline & Cryptantha crassipes & {$[64]$} \\
\hline & Cryptantha clevelandii & {$[63]$} \\
\hline & Cryptantha flava & {$[63]$} \\
\hline & Cryptantha leiocarpa & [63] \\
\hline & Cryptantha thyrsiflora & [63] \\
\hline & Cryptantha virgata & {$[63]$} \\
\hline & Echium pininana & [95] \\
\hline
\end{tabular}


Table 2. Cont.

\begin{tabular}{|c|c|c|}
\hline Compounds & Sources & References \\
\hline \multirow[t]{19}{*}{ 7-Acetylintermedine } & Amsinckia carinata & {$[42]$} \\
\hline & Amsinckia menziesii var. intermedia & [42] \\
\hline & Amsinckia tessellata & {$[46]$} \\
\hline & Anchusa arvensis & {$[40]$} \\
\hline & Borago officinalis & {$[55]$} \\
\hline & Cryptantha cana & {$[63]$} \\
\hline & Cryptantha crassipes & {$[64]$} \\
\hline & Cryptantha flava & [63] \\
\hline & Cryptantha thyrsiflora & [63] \\
\hline & Cryptantha virgata & {$[63]$} \\
\hline & Lappula myostis & {$[35]$} \\
\hline & Lithospermum canescens & {$[153,154]$} \\
\hline & Onosma alborosea & {$[161]$} \\
\hline & Onosma arenaria pennina & [161] \\
\hline & Onosma stellulatum & [167] \\
\hline & Pulmonaria obscura & {$[168]$} \\
\hline & Symphytum officinale & {$[171,172,179]$} \\
\hline & Symphytum peregrinum & {$[27]$} \\
\hline & Symphytum $\times$ uplandicum & {$[180]$} \\
\hline 5'-Acetyllasiocarpine & Heliotropium hirsutissimum & [129] \\
\hline 7-Acetyl-9-latifolylretronecine & Hackelia californica & [101] \\
\hline 3'-Acetyllithosenine & Lithospermum officinale & [156] \\
\hline \multirow[t]{13}{*}{ 3'-Acetyllycopsamine } & Amsinckia grandiflora & {$[42]$} \\
\hline & Amsinckia lunaris & {$[42]$} \\
\hline & Amsinckia menziesii & {$[45]$} \\
\hline & Amsinckia menziesii var. intermedia & {$[42]$} \\
\hline & Amsinckia tessellata & {$[46]$} \\
\hline & Amsinckia tessellata var. tessellata & {$[42]$} \\
\hline & Cerinthe glabra & {$[40]$} \\
\hline & Cryptantha cana & {$[63]$} \\
\hline & Cryptantha crassipes & {$[64]$} \\
\hline & Cryptantha flava & {$[63]$} \\
\hline & Cryptantha jamesii & {$[66]$} \\
\hline & Cryptantha thyrsiflora & {$[63]$} \\
\hline & Echium lycopsis (E. plantagineum) & [93] \\
\hline \multirow[t]{10}{*}{ 7-Acetyllycopsamine } & Amsinckia grandiflora & {$[42]$} \\
\hline & Amsinckia menziesii & {$[45]$} \\
\hline & Amsinckia menziesii var. intermedia & {$[42]$} \\
\hline & Amsinckia tessellata & {$[46]$} \\
\hline & Amsinckia tessellate var. tessellata & {$[42]$} \\
\hline & Anchusa arvensis & {$[40]$} \\
\hline & Anchusa hispida & {$[40]$} \\
\hline & Anchusa officinalis & {$[48]$} \\
\hline & Borago officinalis & {$[55]$} \\
\hline & Cerinthe glabra & {$[40]$} \\
\hline
\end{tabular}


Table 2. Cont.

\begin{tabular}{|c|c|c|}
\hline Compounds & Sources & References \\
\hline \multirow[t]{23}{*}{ 7-Acetyllycopsamine } & Cryptantha cana & [63] \\
\hline & Cryptantha crassipes & [64] \\
\hline & Cryptantha flava & [63] \\
\hline & Cryptantha jamesii & {$[66]$} \\
\hline & Cryptantha thyrsiflora & {$[63]$} \\
\hline & Cryptantha virgata & [63] \\
\hline & Echium horridum & {$[90]$} \\
\hline & Echium hypertropicum & {$[92]$} \\
\hline & Echium humile & [91] \\
\hline & Echium rauwolfi & {$[90]$} \\
\hline & Lappula myostis & [35] \\
\hline & Lappula spinocarpos & {$[40]$} \\
\hline & Lithospermum canesens & {$[153,154]$} \\
\hline & Onosma alborosea & {$[161]$} \\
\hline & Onosma arenaria & [163] \\
\hline & Onosma arenaria pennina & {$[161]$} \\
\hline & Pulmonaria obscura & [168] \\
\hline & Symphytum bohemium & [173] \\
\hline & Symphytum officinale & {$[171,172,179]$} \\
\hline & Symphytum peregrinum & [27] \\
\hline & Symphytum tanaiense & [173] \\
\hline & Symphytum tubertosum & {$[28]$} \\
\hline & Symphytum $\times$ uplandicum & {$[180]$} \\
\hline 7-Acetyl-9-(2-methylbutyryl) retronecine & Onosma arenaria & {$[163]$} \\
\hline \multirow[t]{2}{*}{ 3'-Acetylmyscorpine } & Amsinckia menziesii var. intermedia & {$[42]$} \\
\hline & Amsinckia retrosa & {$[42]$} \\
\hline 7-Acetylretronecine & Onosma arenaria & [163] \\
\hline \multirow[t]{2}{*}{ 3'-Acetylrinderine } & Cynoglossum columnae & [69] \\
\hline & Heliotropium transplinum & [144] \\
\hline 7-Acetyl-9-sarracinoyl retronecine & Alkanna tinctoria & {$[40]$} \\
\hline 7-Acetylscorpioidine & Myosotis scorpioides & [159] \\
\hline 3'-Acetylsupinine & Onosma arenaria & [163] \\
\hline 3'-Acetyltessellatine & Amsinckia tessellata var. tessellata & {$[42]$} \\
\hline \multirow[t]{3}{*}{ 9-Acetytessellatine } & Amsinckia grandifkora & {$[42]$} \\
\hline & Amsinckia lunaris & [42] \\
\hline & Amsinckia tessellata var. tessellata & {$[42]$} \\
\hline 3'-Acetyltrachelanthamine & Heliotropium floridum & [128] \\
\hline 9-Acetyltrachelanthamine & Anchusa arvensis & {$[40]$} \\
\hline 3'-Acetylviridiflorine & Amsinckia tessellata var. gloriosa & [42] \\
\hline \multirow[t]{2}{*}{ 9-(3'-Acetyl)viridiflory retronecine } & $\begin{array}{l}\text { Heliotropium curassavicum var. } \\
\text { argentinum }\end{array}$ & [119] \\
\hline & $\begin{array}{l}\text { Heliotropium curassavicum var. } \\
\text { curassavicum }\end{array}$ & [119] \\
\hline \multirow[t]{2}{*}{ 9-(3'-Acetylviridifloryl) turniforcidine } & A. douglasiana & {$[42]$} \\
\hline & A. tessellata var. tessellata & [42] \\
\hline
\end{tabular}


Table 2. Cont.

\begin{tabular}{|c|c|c|}
\hline Compounds & Sources & References \\
\hline 7-Acetylvulgarine & Echium vulgare & [11] \\
\hline \multirow[t]{20}{*}{ Amabiline } & Amsinckia douglasina & [42] \\
\hline & Amsinckia eastwoodiae & {$[42]$} \\
\hline & Amsinckia grandiflora & [42] \\
\hline & Amsinckia lycopsoides & [42] \\
\hline & $\begin{array}{l}\text { Amsinckia lycopsodes menziesii } \\
\text { var. intermedium }\end{array}$ & [42] \\
\hline & Amsinckia menziesii var intermedium & [42] \\
\hline & Amsinckia retrosa & [42] \\
\hline & Amsinckia tessellata var. gloriosa & [42] \\
\hline & Amsinckia tessellata var. tessellata. & [42] \\
\hline & Asperugo procumbens & [47] \\
\hline & Borago officinalis & [55] \\
\hline & Cryptantha confertiflora & [63] \\
\hline & Cryptantha crassipes & {$[64]$} \\
\hline & Cryptantha virginensis & [63] \\
\hline & Cynoglossum amabile & {$[67,68]$} \\
\hline & Cynoglossum glochidiatum & [78] \\
\hline & Heliotropium spathulatum & [140] \\
\hline & Lappula spinocarpos & [40] \\
\hline & Lindelofia angustiflora & {$[78]$} \\
\hline & Neatostema apulum & [160] \\
\hline \multirow[t]{2}{*}{ Anadoline } & Symphytum orientale & {$[28]$} \\
\hline & Symphytum tuberosum & {$[28]$} \\
\hline $\begin{array}{l}\text { 7a-Angeloyl-1-chloromethy-1,2- } \\
\text { dihydropyrrolizidine }\end{array}$ & Cynoglossum creticum & {$[70]$} \\
\hline 7-Angeloyl-9-(2,3-dihydroxybutyryl)heliotridine & Cynoglossum officinale & [67] \\
\hline \multirow[t]{4}{*}{ 7-Angeloyl-9-(2,3-dihydroxybutyryl)retronecine } & Echium horridum & {$[90]$} \\
\hline & Echium rauwolfii & {$[90]$} \\
\hline & Echium setosum & [96] \\
\hline & Onosma arenaria & [163] \\
\hline \multirow[t]{2}{*}{ 7-Angeloyl-9-(2,3-dihydroxypropanoyl)retronecine } & Alkanna orientalis & [40] \\
\hline & Alkanna tinctoria & [40] \\
\hline 7-Angeloyl-1-formyl-6,7-dihydro-5H-pyrrolizidine & Cynoglossum officinale & [67] \\
\hline \multirow[t]{2}{*}{ 7-Angeloyl-9-(hydroxypropenoyl)retronecine } & Alkanna orientalis & {$[40]$} \\
\hline & Alkanna tinctoria & [40] \\
\hline 7-Angeloylechinatine & Cynoglossum officinale & [67] \\
\hline \multirow[t]{8}{*}{ 7-Angeloylheliotridine } & Anchusa hispida (Gastrocotyle hispida) & {$[40]$} \\
\hline & Anchusa milleri & {$[40]$} \\
\hline & Cynoglossum creticum & {$[70,72]$} \\
\hline & Cynoglossum latifolium & [79] \\
\hline & Cynoglossum officinale & {$[67,84]$} \\
\hline & Heliotropium curassavicum & [117] \\
\hline & Heliotropium supinum & {$[27,143]$} \\
\hline & Lappula spinocarpos & {$[40]$} \\
\hline
\end{tabular}


Table 2. Cont.

\begin{tabular}{|c|c|c|}
\hline Compounds & Sources & References \\
\hline \multirow[t]{3}{*}{ 7-Angeloylheliotridine } & Paracaryum intermedium & {$[40]$} \\
\hline & Paracaryum regulosum & {$[40]$} \\
\hline & Rindera austroechinata & [57] \\
\hline \multirow[t]{5}{*}{ 7-Angeloylheliotrine } & Heliotropium circinatum & [114] \\
\hline & Heliotropium digynum (H. luteum) & [121] \\
\hline & Heliotropium eichwaldii & [124] \\
\hline & Heliotropium europaeum & [127] \\
\hline & Rindera umbellata & [169] \\
\hline \multirow[t]{2}{*}{ 7-Angeloyllycopsamine } & Echium horridum & [90] \\
\hline & Echium rauwolfii & {$[90]$} \\
\hline \multirow[t]{2}{*}{ 7-Angeloyl-9-(2-methylbutyryl)heliotridine } & Cynoglossum creticum & [70] \\
\hline & Cynoglossum offocinale & [67] \\
\hline \multirow[t]{2}{*}{ 7-Angeloyl-9-(2-methylbutyryl)retronecine } & Echium horridum & [90] \\
\hline & Echium rauwolfii & {$[90]$} \\
\hline \multirow[t]{18}{*}{ 7-Angeloylretronecine } & Alkanna orientalis & {$[40,41]$} \\
\hline & Alkanna tinctoria (A. tuberculata) & {$[40,184]$} \\
\hline & Arnebia decumbens & {$[52]$} \\
\hline & Arnebia euchroma & [53] \\
\hline & Cerinthe minor & [60] \\
\hline & Cryptantha fendleri & [63] \\
\hline & Cryptantha leiocarpa & [63] \\
\hline & Echium amoenum & [87] \\
\hline & Echium glomeratum & [89] \\
\hline & Echium horridum & [90] \\
\hline & Echium rauwolfii & {$[90]$} \\
\hline & Echium setosum & [96] \\
\hline & Echium simplex & [162] \\
\hline & Echium vulgare & [96] \\
\hline & Eritrichium rupestre & {$[60]$} \\
\hline & Hackelia longituba & [104] \\
\hline & Heliotropium bursiferum & [113] \\
\hline & Symphytum tuberosum & {$[162]$} \\
\hline \multirow[t]{13}{*}{ 9-Angeloylretronecine } & Alkanna orientalis & {$[40,41]$} \\
\hline & Arnebia decumbens & {$[52]$} \\
\hline & Cryptantha fendleri & [63] \\
\hline & Cryptantha leiocarpa & [63] \\
\hline & Cynoglossum amabilie & [67] \\
\hline & Echium glomaratum & [89] \\
\hline & Echium hypertropicum & [92] \\
\hline & Echium setosum & [96] \\
\hline & Echium simplex & [162] \\
\hline & Echium vulgare & [96] \\
\hline & Hackelia longituba & [104] \\
\hline & Messerchimidia sibrica & [28] \\
\hline & Myosotis sylvatica & {$[25]$} \\
\hline
\end{tabular}


Table 2. Cont.

\begin{tabular}{|c|c|c|}
\hline Compounds & Sources & References \\
\hline 7-Angeloylrinderine & Cynoglossun officinale & {$[67]$} \\
\hline 9-Angeloyltrachelanthamidine & Anchusa arvensis & [40] \\
\hline 7-Angeloyl-9-(+)-trachelanthylheliotridine & Rindera umbellata & [169] \\
\hline \multirow[t]{5}{*}{ 9-Angeloyl-7-viridiflorylretronecine } & Cerinthe minor & {$[60]$} \\
\hline & Cynoglossum amabile & {$[60]$} \\
\hline & Cynoglossum clandestinum & [60] \\
\hline & Echium tuberculatum & {$[60]$} \\
\hline & Eritrichium rupestre & {$[60]$} \\
\hline \multirow[t]{3}{*}{ Asperumine } & Echium vulgare & [98] \\
\hline & Symphytum asperum & [172] \\
\hline & Symphytum caucasium & [174] \\
\hline 9-(Butyryl-2-ene) supinidine & Onosma arenaria & [163] \\
\hline Canescine & Lithospermum canescens & {$[153,154]$} \\
\hline Canescenine & Lithospermum canescens & {$[153,154]$} \\
\hline \multirow[t]{3}{*}{ Carategine } & Lindelofia tschimganica & {$[27]$} \\
\hline & Rindera oblongifolia & [152] \\
\hline & Solanthus karateginus & {$[151]$} \\
\hline \multirow[t]{2}{*}{ Coromandaline } & Heliotropium curassavicum & {$[117,118]$} \\
\hline & Heliotropium spathulatum & {$[140]$} \\
\hline \multirow[t]{2}{*}{ Coromandalinine } & Heliotropium curassavicum & [117] \\
\hline & Heliotropium spathulatum & [140] \\
\hline Cryptanthine & Cryptantha utahensis & {$[65]$} \\
\hline Curassanecine & Heliotropium curassavicum & [117] \\
\hline \multirow[t]{3}{*}{ Curassavine } & Anchusa officinalis & [49] \\
\hline & Heliotropium curassavicum & [117] \\
\hline & Heliotropium spathulatum & [140] \\
\hline \multirow[t]{2}{*}{ Curassavinine } & Heliotropium curassavicum & [117] \\
\hline & Heliotropium spathulatum & {$[140]$} \\
\hline 9-Curassavorylheliotridine & Anchusa milleri & [40] \\
\hline \multirow[t]{3}{*}{ Cynaustrine } & Cynoglossum australe & [68] \\
\hline & Cynoglossum lanceolatum & {$[78]$} \\
\hline & Cynoglossum montanum & [81] \\
\hline \multirow[t]{5}{*}{ Cynaustraline } & Amsinckia douglasiana & [42] \\
\hline & Cynoglossum australe & [68] \\
\hline & Cynoglossum furcatum & [73] \\
\hline & Cynoglossum lanceolatum & {$[78]$} \\
\hline & Cynoglossum montanum & [81] \\
\hline Cynoglossamine & Cynoglossum creticum & {$[72]$} \\
\hline Dehydroheliotrine & Heliotropium europaeum & [185] \\
\hline 5-Deoxylasiocarpine & Heliotropium dissitiflorum & [123] \\
\hline \multirow[t]{3}{*}{ 3',7-Diacetylintermedine } & Amsinckia menziesii var. intermedia & [42] \\
\hline & Amsinckia tessellata & {$[46]$} \\
\hline & Anchusa arvensis & [40] \\
\hline \multirow[t]{2}{*}{ 3',7-Diacetyllycopsamine } & Amsinckia menziesii & {$[45]$} \\
\hline & Amsinckia tessellata & [46] \\
\hline
\end{tabular}


Table 2. Cont.

\begin{tabular}{|c|c|c|}
\hline Compounds & Sources & References \\
\hline \multirow[t]{2}{*}{ 3',7-Diacetyllycopsamine } & Anchusa arvensis & {$[40]$} \\
\hline & Cerinthe glabra & [59] \\
\hline 3',9-Diacetyltessellatine & Amsinckia tessellata var. tessellata & [42] \\
\hline 5,6-Dihydro-7,9-dimethoxy-7H-pyrrolizine & Onosma arenaria & [163] \\
\hline Dihydroechinatine & Onosma stellulatum & [167] \\
\hline \multirow[t]{3}{*}{ thero-2",3"-Dihydroxyechiumine } & Cryptantha clevelandii & {$[63,65]$} \\
\hline & Cryptantha inequata & \\
\hline & Cryptantha leiocarpa & {$[63]$} \\
\hline \multirow[t]{2}{*}{ Dihydroxytriangularine } & Alkanna orientalis & {$[40,41]$} \\
\hline & Alkanna tinctoria & {$[40,41]$} \\
\hline \multirow[t]{2}{*}{ Dihydroxytriangularicine } & Alkanna orientalis & [40] \\
\hline & Alkanna tinctoria & {$[40]$} \\
\hline \multirow[t]{4}{*}{ Echihumiline } & Echium hypertropicum & [92] \\
\hline & Echium humile & [91] \\
\hline & Echium vulgare & [96] \\
\hline & Onosma leptantha & [166] \\
\hline \multirow[t]{25}{*}{ Echimidine } & Arnebia hispidissima & [54] \\
\hline & Cryptantha inequata & {$[65]$} \\
\hline & Echium amoenum & [87] \\
\hline & Echium angustifolium & [88] \\
\hline & Echium horridum & {$[90]$} \\
\hline & Echium humile & [91] \\
\hline & Echium hypertropicum & [92] \\
\hline & Echium italicum & {$[28]$} \\
\hline & Echium lycopsis (E. plantagineum) & [94] \\
\hline & Echium pininana & [95] \\
\hline & Echium rauwolfii & {$[90]$} \\
\hline & Echium sericeum & {$[54]$} \\
\hline & Echium. setosum & [96] \\
\hline & Echium stenosiphon subsp. stenosiphon & {$[92]$} \\
\hline & Echium tuberculatum & {$[60]$} \\
\hline & Echium vulgare & {$[11,96]$} \\
\hline & Echium wildpretti & [99] \\
\hline & Onosma stellulatum & {$[60,167]$} \\
\hline & Symphytum aintabicum & [170] \\
\hline & Symphytum asperum & [171] \\
\hline & Symphytum bohemium & [173] \\
\hline & Symphytum caucasium & [174] \\
\hline & Symphytum consolidum & [175] \\
\hline & Symphytum sylvaticum & [170] \\
\hline & Symphytum tuberosum & {$[162]$} \\
\hline \multirow[t]{2}{*}{ Echimidine isomer (tigloyl) } & Echium amoenum & {$[87]$} \\
\hline & Echium horridum & {$[90]$} \\
\hline
\end{tabular}


Table 2. Cont.

\begin{tabular}{|c|c|c|}
\hline Compounds & Sources & References \\
\hline \multirow{3}{*}{ Echimidine isomer (tigloyl) } & Echium rauwolfii & {$[90]$} \\
\hline & Echium setosum & [96] \\
\hline & Echium vulgare & [96] \\
\hline \multirow[t]{3}{*}{ Echimiplatine } & Cryptantha crassipes & [64] \\
\hline & Echium plantagineum & [93] \\
\hline & Echium vulgare & [11] \\
\hline \multirow[t]{35}{*}{ Echinatine } & Asperugo procumbens & [47] \\
\hline & Cynoglossum amabile & {$[67,68]$} \\
\hline & Cynoglossum columnae & {$[69]$} \\
\hline & Cynoglossum creticum & [70-72] \\
\hline & Cynoglossum furcatum (C. zeylanicum) & {$[74,75]$} \\
\hline & Cynoglossum germanicum & {$[77]$} \\
\hline & Cynoglossum macrostylum & {$[80]$} \\
\hline & Cynoglossum montanum & [81] \\
\hline & Cynoglossum nervosum & [82] \\
\hline & Cynoglossum officinale & {$[67,84]$} \\
\hline & Cynoglossum pictum & [85] \\
\hline & Heliotropium circinatum & [114] \\
\hline & Heliotropium indicum & [130] \\
\hline & Heliotropium suaveolens & [29] \\
\hline & Heliotropium supinum & {$[27,143]$} \\
\hline & Lappula glochidiata & {$[146]$} \\
\hline & Lindelofia longiflora & [149] \\
\hline & Lindelofia spectabilis & [124] \\
\hline & Lindelofia stylosa & [135] \\
\hline & Moltikiopsis ciliata & [108] \\
\hline & Paracaryum regulosum & {$[40]$} \\
\hline & Paracynoglossum imeretium & [29] \\
\hline & Rindera austroechinata & {$[57,152]$} \\
\hline & Rindera baldschuanica & {$[152]$} \\
\hline & Rindera cyclodonata & {$[120]$} \\
\hline & Rindera echinata & {$[120]$} \\
\hline & Rindera oblogifolia & {$[152]$} \\
\hline & Rindera umbellata & [169] \\
\hline & Solenanthus circinnatus & {$[151]$} \\
\hline & Solenanthus coronatus & [135] \\
\hline & Solenanthus karateginus & {$[151]$} \\
\hline & Symphytum asperum & [172] \\
\hline & Symphytum caucasium & {$[174]$} \\
\hline & Symphytum officinale & [179] \\
\hline & Tournefortia sogdiana & {$[57]$} \\
\hline \multirow[t]{4}{*}{ Echiumine } & Amsinckia carinata & [42] \\
\hline & Amsinckia eastwoodiae & [42] \\
\hline & Amsinckia hispida & [44] \\
\hline & Amsinckia intermedia & [44] \\
\hline
\end{tabular}


Table 2. Cont.

\begin{tabular}{|c|c|c|}
\hline Compounds & Sources & References \\
\hline \multirow[t]{6}{*}{ Echiumine } & Amsinckia lycopsoides & [44] \\
\hline & Amsinckia menziesii var. intermedia & {$[42]$} \\
\hline & Cryptantha clevelandii & [63] \\
\hline & Cryptantha crassipes & [64] \\
\hline & Cryptantha leiocarpa & [63] \\
\hline & Echium lycopsis & [94] \\
\hline Echiupine & Echium pininana & [95] \\
\hline \multirow[t]{3}{*}{ Echiuplatine } & Cryptantha inequata & [65] \\
\hline & Echium plantagineum & [93] \\
\hline & Echium vulgare & [11] \\
\hline Echivulgarine & Echium vulgare & [11] \\
\hline Ehretinine & Ehretia aspera & {$[100]$} \\
\hline \multirow[t]{2}{*}{ 2",3"-Epoxyechiumine } & Cryptantha clevelandii & [63] \\
\hline & Cryptantha leiocarpa & [63] \\
\hline \multirow[t]{2}{*}{ Erythro-2",3"-chloro-2"-hydroxyechiumine } & Cryptantha clevelandii & [63] \\
\hline & Cryptantha leiocarpa & [63] \\
\hline 7-Epi-echimiplatine & Onosma erecta & [69] \\
\hline 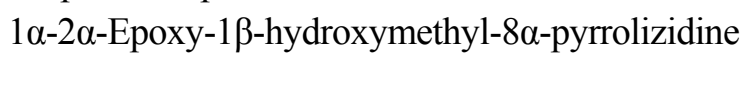 & $\begin{array}{l}\text { Heliotropium transalpinum var. } \\
\text { transalpinum }\end{array}$ & [145] \\
\hline \multirow[t]{13}{*}{ Europine } & Heliotropium arbainense & [71] \\
\hline & Heliotropium bacciferum & {$[108,109]$} \\
\hline & Heliotropium bovi & {$[110]$} \\
\hline & Heliotropium circinatum & [114] \\
\hline & Heliotropium crassifolium & [115] \\
\hline & Heliotropium digynum (H.luteum) & [121] \\
\hline & Heliotropium dissitiflorum & [123] \\
\hline & Heliotropium esfandiarii & [125] \\
\hline & Heliotropium europaeum & {$[126,127,186]$} \\
\hline & Heliotropium hirsutissinum & [129] \\
\hline & Heliotropium marifolium & [29] \\
\hline & Heliotropium maris mortui & [71] \\
\hline & Heliotropium rotundifolium & {$[137,138]$} \\
\hline Floridanine & Cordia sinensis & [62] \\
\hline Floridimine & Heliotropium floridum & [128] \\
\hline Floridine & Heliotropium floridum & [128] \\
\hline Floridinine & Heliotropium floridum & [128] \\
\hline \multirow[t]{3}{*}{ Furcatine } & Amsinckia carinata & [42] \\
\hline & Amsinckia furacata & [42] \\
\hline & Amsinckia vernicosa & [42] \\
\hline Hackelidine & Hackelia californica & [102] \\
\hline \multirow[t]{6}{*}{ Heleurine } & Heliotropium bacciferum & [109] \\
\hline & Heliotropium circinatum & [114] \\
\hline & Heliotropium disciforme & {$[122]$} \\
\hline & Heliotropium europaeum & {$[126,127,186]$} \\
\hline & Heliotropium hirsutissimum & [129] \\
\hline & Heliotropium indicum & [131] \\
\hline
\end{tabular}


Table 2. Cont.

\begin{tabular}{|c|c|c|}
\hline Compounds & Sources & References \\
\hline Helibracteatine & Heliotropium bracteatum & {$[112]$} \\
\hline Helibractinecine & Heliotropium bracteatum & [111] \\
\hline Helibracteatinecine & Heliotropium bracteatum & [112] \\
\hline Helibracteatinine & Heliotropium bracteatum & [112] \\
\hline Heliofoline & Heliotropium ovalifolium & [136] \\
\hline Helindicine & Heliotropium indicum & [130] \\
\hline Heliocoromandaline & Heliotropium curassavicum & {$[117]$} \\
\hline Heliocurassavine & Heliotropium curassavicum & [117] \\
\hline Heliocurassavicine & Heliotropium curassavicum & {$[117]$} \\
\hline Heliocurassavinine & Heliotropium curassavicum & [117] \\
\hline Heliospathine & Heliotropium spathulatum & {$[140]$} \\
\hline \multirow[t]{2}{*}{ Heliospathuline } & Heliotropium spathulatum & [140] \\
\hline & Onosma stellulatum & {$[167]$} \\
\hline \multirow[t]{13}{*}{ Heliosupine } & Cynoglossium creticum & [70-72] \\
\hline & Cynoglossium macrostylum & {$[80]$} \\
\hline & Cynoglossium montatum & {$[81]$} \\
\hline & Cynoglossium officinale & {$[67,83,84]$} \\
\hline & Cynoglossium pictum & {$[85]$} \\
\hline & Cynoglossium viridiforum & {$[86]$} \\
\hline & Heliotropium supinum & {$[27,143]$} \\
\hline & Myosotis sylvatica & {$[25]$} \\
\hline & Paracaryum regulosum & {$[90]$} \\
\hline & Paracynoglossum imeretium & [29] \\
\hline & Rindera umbellata & [169] \\
\hline & Symphytum asperum & {$[171]$} \\
\hline & Symphytum officinale & [179] \\
\hline \multirow[t]{2}{*}{ Heliotridine } & Anchusa milleri & {$[90]$} \\
\hline & Onosma heterophyllum & [165] \\
\hline $\begin{array}{l}\text { Heliotridine 2S-hydroxy-2S(1S-hydroxyethyl0-4- } \\
\text { methyl-pentanoyl ester }\end{array}$ & Anchusa strigosa & {$[50]$} \\
\hline \multirow[t]{15}{*}{ Heliotrine } & Arenbia decumbens & {$[52]$} \\
\hline & Cynoglossum nervosum & {$[82]$} \\
\hline & Heliotropium acutifolium & {$[105]$} \\
\hline & H. arbainense & {$[71]$} \\
\hline & H. bacciferum & {$[108,109]$} \\
\hline & Heliotropium circinatum & [114] \\
\hline & Heliotropium dasycarpum & {$[120]$} \\
\hline & Heliotropium digynum & {$[121]$} \\
\hline & Heliotropium disciforme & {$[122]$} \\
\hline & Heliotropium dissitiflorum & {$[123]$} \\
\hline & Heliotropium eichwaldii & {$[124]$} \\
\hline & Heliotropium europaeum & {$[127,185,186]$} \\
\hline & Heliotropium hirsutissimum & [129] \\
\hline & Heliotropium indicum & {$[130,131]$} \\
\hline & Heliotropium lasiocarpum & [29] \\
\hline
\end{tabular}


Table 2. Cont.

\begin{tabular}{|c|c|c|}
\hline Compounds & Sources & References \\
\hline \multirow[t]{10}{*}{ Heliotrine } & Heliotropium marifolium & {$[29]$} \\
\hline & Heliotropium olgae & [135] \\
\hline & Heliotropium popovii subsp. gillianum & {$[28]$} \\
\hline & Heliotropium rotundifolum & {$[137,138]$} \\
\hline & Heliotropium suaveolens & [29] \\
\hline & Heliotropium supinum & {$[27,143]$} \\
\hline & Heliotropium transoxanum & {$[27]$} \\
\hline & Molyikiopsis ciliate & [108] \\
\hline & (Lithospermum callosum) & \\
\hline & Symphytum caucasium & [174] \\
\hline Heliovicine & Heliotropium spathulatum & [140] \\
\hline Heliscabine & Heliotropium scabrum & [139] \\
\hline Hydroxymyoscorpine & Echium pininana & [95] \\
\hline Ilamine & Heliotropium crassifolium & [115] \\
\hline \multirow[t]{2}{*}{ Incanine } & Heliotropium olgae & [29] \\
\hline & Trichodesma incanum & [46] \\
\hline \multirow[t]{7}{*}{ Indicine } & Heliotropium amplexicaule & [29] \\
\hline & Heliotropium arborescens & {$[107]$} \\
\hline & (H. pruvianum) & \\
\hline & Heliotropium indicum & {$[130,131]$} \\
\hline & Heliotropium marifolium & [29] \\
\hline & Heliotropium transalpinum & [144] \\
\hline & Messerschmidia argentea & {$[158]$} \\
\hline Indicinine & Heliotropium indicum & {$[130,131]$} \\
\hline Isoechinatine & Cynoglossum furcatum (C. zeylanicum) & {$[75]$} \\
\hline Isolycopsamine & Heliotropium keralense & [190] \\
\hline Isoretronocanol (or its isomer) & Omphalodes verna & {$[27]$} \\
\hline \multirow[t]{2}{*}{ 9-(3'-Isovaleryl)viridiflory retronecine } & $\begin{array}{l}\text { Heliotropium curassavicum var. } \\
\text { argentinum }\end{array}$ & [119] \\
\hline & $\begin{array}{l}\text { Heliotropium curassavicum var. } \\
\text { curassavicum }\end{array}$ & [119] \\
\hline \multirow[t]{13}{*}{ Intermedine } & Amsinckia carinata & [42] \\
\hline & Amsinckia douglasiana & [42] \\
\hline & Amsinckia eastwoodiae & [42] \\
\hline & Amsinckia furacata & [42] \\
\hline & Amsinckia. grandiflora & [42] \\
\hline & Amsinckia hispida & [44] \\
\hline & Amsinckia intermedia & [44] \\
\hline & Amsinckia lunaris & [42] \\
\hline & Amsinckia lycopsoides & {$[42,44]$} \\
\hline & $\begin{array}{l}\text { Amsinckia lycopsoides } \times \text { menziesii } \\
\text { var. intermedia }\end{array}$ & {$[42]$} \\
\hline & Amsinckia menziesii & {$[45]$} \\
\hline & Amsinckia menziesii var. intermedia & [42] \\
\hline & Amsinckia retrosa & [42] \\
\hline
\end{tabular}


Table 2. Cont.

\begin{tabular}{|c|c|c|}
\hline Compounds & Sources & References \\
\hline \multirow[t]{34}{*}{ Intermedine } & Amsinckia spectabilis var. microcarpa & {$[42]$} \\
\hline & Amsinckia spectabilis var. spectabilis & [42] \\
\hline & Amsinckia spectabilis var. nicolai & [42] \\
\hline & Amsinckia tessellata & {$[46]$} \\
\hline & Amsinckia tessellata var. gloriosa & [42] \\
\hline & Amsinckia tessellata var. tessellata & [42] \\
\hline & Amsinckia vernicosa & [42] \\
\hline & Anchusa arvensis (Lycopsis arvensis) & [40] \\
\hline & Anchusa hispidia (Gastrocotyle hispidia) & {$[40]$} \\
\hline & Anchusa officinalis & [48] \\
\hline & Borago officinalis & {$[55]$} \\
\hline & Cerinthe minor & [61] \\
\hline & Cryptantha cana & [63] \\
\hline & Cryptantha clevelandii & [63] \\
\hline & Cryptantha confertiflora & [63] \\
\hline & Cryptantha flava & [63] \\
\hline & Cryptantha inequata & {$[65]$} \\
\hline & Cryptantha jamesii & {$[66]$} \\
\hline & Cryptantha leiocarpa & [63] \\
\hline & Cryptantha thyrsiflora & [63] \\
\hline & Cryptantha virgata & [63] \\
\hline & Cryptantha virginiensis & [63] \\
\hline & Heliotropium keralense & [133] \\
\hline & Heliotropium transalpinum & [144] \\
\hline & Lappula myostis & [35] \\
\hline & Lappula spinocarpas & [40] \\
\hline & Lithospermum erythrorhizon & [155] \\
\hline & Mertensia ciliate & [157] \\
\hline & Onosma alborosea & [161] \\
\hline & Onosma arenaria pennina & [161] \\
\hline & Pulmonaria obscura & [168] \\
\hline & Symphytum peregrinum & {$[27]$} \\
\hline & Symphytum $\times$ uplandicum & [180] \\
\hline & Trichodesma africanum & [71] \\
\hline Lactodine & Cynoglossum furcatum & {$[76,187]$} \\
\hline \multirow[t]{10}{*}{ Lasiocarpine } & Heliotropium arbainense & {$[71]$} \\
\hline & Heliotropium bovei & [110] \\
\hline & Heliotropium circinatum & [114] \\
\hline & Heliotropium digynum & [121] \\
\hline & Heliotropium eichwaldii & [124] \\
\hline & Heliotropium europaeum & {$[126,127,186]$} \\
\hline & Heliotropium hirsutissimum & [129] \\
\hline & Heliotropium indicum & {$[130,131]$} \\
\hline & Heliotropium lasiocarpum & [29] \\
\hline & Heliotropium marifolium & [29] \\
\hline
\end{tabular}


Table 2. Cont.

\begin{tabular}{|c|c|c|}
\hline Compounds & Sources & References \\
\hline \multirow[t]{6}{*}{ Lasiocarpine } & Heliotropium maris mortui & {$[137]$} \\
\hline & Heliotropium rotundifolium & {$[137,138]$} \\
\hline & Heliotropium suaveolens & [29] \\
\hline & Lappula intrmedia & {$[27]$} \\
\hline & Symphytum caucasium & {$[174]$} \\
\hline & Symphytum officinale & {$[177]$} \\
\hline \multirow[t]{6}{*}{ Latifoline } & Cryptantha fendleri & {$[63]$} \\
\hline & Cynoglossum latifolium & [79] \\
\hline & Hackelia californica & {$[101]$} \\
\hline & Hackelia floribunda & [103] \\
\hline & Hackelia longituba & [104] \\
\hline & Lindelofia stylosa & {$[27]$} \\
\hline 9-Latifolylretronecine & Hackelia californica & {$[101]$} \\
\hline \multirow[t]{5}{*}{ Leptanthine } & Cryptantha crassipes & [64] \\
\hline & Echium plantagineum & {$[93]$} \\
\hline & Echium vulgare & [11] \\
\hline & Onosma leptantha & {$[166]$} \\
\hline & Onosma stellulatum & {$[167]$} \\
\hline \multirow[t]{6}{*}{ Lindelofine } & Amsickia menzesii var. intermedia & {$[42]$} \\
\hline & Amsickia spectabilis var. microcarpa & [42] \\
\hline & Amsickia spectabilis var. spectabilis & {$[42]$} \\
\hline & Amsickia spectabilis var. nicola & {$[42]$} \\
\hline & Lindelofia anchusoides (L. macrostyle) & {$[28]$} \\
\hline & Rindera umbellata & [169] \\
\hline Lindelofamine & Lindelofia anchusoides (L. macrostyle) & {$[28]$} \\
\hline \multirow[t]{15}{*}{ Lindelofidine } & Heliotropium angiospermum & {$[106]$} \\
\hline & Heliotropium confertifolum & {$[106]$} \\
\hline & Heliotropium curassavicum & {$[106]$} \\
\hline & Heliotropium foliosissimum & {$[106]$} \\
\hline & Heliotropium fruticosum & {$[106]$} \\
\hline & Heliotropium gregii & {$[106]$} \\
\hline & Heliotropium indicum & {$[106,116]$} \\
\hline & Heliotropium molle & {$[106]$} \\
\hline & Heliotropium procumbens & {$[106]$} \\
\hline & Heliotropium queretaroanum & {$[106]$} \\
\hline & Heliotropium spathulatum & [116] \\
\hline & Heliotropium sessei & {$[106]$} \\
\hline & Heliotropium racemosum & {$[106]$} \\
\hline & Heliotropium ternatum & {$[106]$} \\
\hline & Heliotropium wigginsii & {$[106]$} \\
\hline Lithosenine & Lithospermum officinale & {$[156]$} \\
\hline \multirow[t]{2}{*}{ Longitubine } & Hackelia californica & {$[101]$} \\
\hline & Hackelia logituba & [104] \\
\hline \multirow[t]{2}{*}{ Lycopsamine } & Amsinckia carinata & {$[42]$} \\
\hline & Amsinckia douglasiana & {$[42]$} \\
\hline
\end{tabular}


Table 2. Cont.

\begin{tabular}{|c|c|c|}
\hline Compounds & Sources & References \\
\hline \multirow[t]{40}{*}{ Lycopsamine } & Amsinckia eastwoodiae & {$[42]$} \\
\hline & Amsinckia furacata & [42] \\
\hline & Amsinckia grandiflora & [42] \\
\hline & Amsinckia hispida & [44] \\
\hline & Amsinckia intermedia & [44] \\
\hline & Amsinckia lunaris & [42] \\
\hline & Amsinckia lycopsoides & {$[42,44]$} \\
\hline & $\begin{array}{l}\text { Amsinckia lycopsoides } \times \text { menziesii } \\
\text { var. } \text { intermedia }\end{array}$ & {$[42]$} \\
\hline & Amsinckia menziesii & {$[45]$} \\
\hline & Amsinckia menziesii var. intermedia & {$[42]$} \\
\hline & Amsinckia retrosa & [42] \\
\hline & Amsinckia spectabilis var. microcarpa & [42] \\
\hline & Amsinckia spectabilis var. spectabilis & [42] \\
\hline & Amsinckia spectabilis var. nicolai & [42] \\
\hline & Amsinckia tessellata & {$[46]$} \\
\hline & Amsinckia tessellata var. gloriosa & [42] \\
\hline & Amsinckia tessellata var. tessellata & [42] \\
\hline & Amsinckia vernicosa & [42] \\
\hline & Anchusa arvensis (Lycopsis arvensis) & [40] \\
\hline & Anchusa hispidia (Gastrocotyle hispidia) & [40] \\
\hline & Anchusa officinalis & [48] \\
\hline & Arnebia decumbens & [52] \\
\hline & Borago officinalis & [55] \\
\hline & Cerinthe glabra & [59] \\
\hline & Cerinthe minor & [61] \\
\hline & Cryptantha cana & [63] \\
\hline & Cryptantha confertiflora & [63] \\
\hline & Cryptantha flava & [63] \\
\hline & Cryptantha inequata & {$[65]$} \\
\hline & Cryptantha jamesii & [66] \\
\hline & Cryptantha thyrsiflora & [63] \\
\hline & Cryptantha virgata & [63] \\
\hline & Cryptantha virginiensis & [63] \\
\hline & Cynoglossum amabile & {$[67]$} \\
\hline & Echium hypertropicum & [92] \\
\hline & Heliotropium transalpinum & [144] \\
\hline & Heliotropium megalanthum & [134] \\
\hline & Heliotropium steudneri & {$[27]$} \\
\hline & Lappula myostis & {$[35]$} \\
\hline & Lappula spinocarpas & [40] \\
\hline & Lithospermum purpureocoeruleum & [60] \\
\hline
\end{tabular}


Table 2. Cont.

\begin{tabular}{|c|c|c|}
\hline Compounds & Sources & References \\
\hline \multirow[t]{17}{*}{ Lycopsamine } & Mertensia bakeri & {$[157]$} \\
\hline & Mertensia ciliate & {$[157]$} \\
\hline & Mertensia sibirica & {$[28]$} \\
\hline & Neatostema apulum & {$[60]$} \\
\hline & Onosma alborosea & {$[161]$} \\
\hline & Onosma arenaria pennina & {$[161]$} \\
\hline & Onosma stellulatum & {$[167]$} \\
\hline & Pulmonaria obscura & {$[168]$} \\
\hline & Symphytum bohemium & [173] \\
\hline & Symphytum grandiflorum & {$[176]$} \\
\hline & Symphytum ibericum & {$[176]$} \\
\hline & Symphytum peregrinum & {$[27]$} \\
\hline & Symphytum officinale & {$[171,177]$} \\
\hline & Symphytum tanaiense & {$[173]$} \\
\hline & Symphytum tuberosum & {$[162,176]$} \\
\hline & Symphytum $\times$ uplandicum & {$[180]$} \\
\hline & Trichodesma africanum & [71] \\
\hline Macrophylline & Cordia myxa & {$[62]$} \\
\hline Macrotamine & Macrotomia echioides & [28] \\
\hline Megalanthonine & Heliotropium megalanthum & {$[134]$} \\
\hline Methyechiuplatine & Cryptantha inequata & {$[65]$} \\
\hline 1-Methylene- $8 \alpha$-pyrrolizidine & Onosma heterophyllum & {$[165]$} \\
\hline \multirow[t]{2}{*}{ 7-(2-Methylbutyryl)retronecine } & Echium humile & [91] \\
\hline & Echium vulgare & [96] \\
\hline 9-(2-Methylbutyryl)retronecine & Echium vulgare & [96] \\
\hline 7-(2-Methylbutyryl)-9-(2,3- & Echium humile & [91] \\
\hline dihydroxybutyryl)retronecine & Echium vulgare & [96] \\
\hline \multirow[t]{4}{*}{ 7-(2-Methylbutyryl)-9-echimidinyl retronecine } & Echium humile & {$[91]$} \\
\hline & Echium hypertropicum & [92] \\
\hline & Echium stenosiphon subsp. stenosiphon & [92] \\
\hline & Echium vulgare & [96] \\
\hline \multirow[t]{2}{*}{ Monocrotaline } & Arnebia hispidissima & {$[54]$} \\
\hline & Lindelofia spectabilis & [124] \\
\hline \multirow[t]{7}{*}{ Myoscorpine } & Amsinckia carinata & {$[42]$} \\
\hline & Amsinckia menziesii var. intermedia & {$[42]$} \\
\hline & Amsinckia retrosa & {$[42]$} \\
\hline & Amsinckia spectabilis var. microcarpa & {$[42]$} \\
\hline & Echium pininana & {$[95]$} \\
\hline & Linelfolia erythrorhizon & {$[155]$} \\
\hline & Myosotis scorpioides & [159] \\
\hline Neocoromandaline & Cynoglossum furcatum & {$[74]$} \\
\hline \multirow[t]{2}{*}{ Neolatifoline } & Cryptantha fendleri & {$[63]$} \\
\hline & Hackelia logituba & [104] \\
\hline Onosmerectine & Onosma erecta & [69] \\
\hline
\end{tabular}


Table 2. Cont.

\begin{tabular}{|c|c|c|}
\hline Compounds & Sources & References \\
\hline$(7 S, 8 R)$ Petranine & Echium glomeratum & [89] \\
\hline$(7 S, 8 S)$ Petranine & Echium glomeratum & [89] \\
\hline Pictumine & Cynoglossum pictum & [28] \\
\hline Platynecine & Cryptantha leiocarpa & [63] \\
\hline $\begin{array}{l}\text { Platynecine } N \text {-oxide } 2 \mathrm{~S} \text {-hydroxy-2S(1S- } \\
\text { hydroxyethyl)-4-methyl-pentanosyl ester }\end{array}$ & Anchusa strigosa & {$[50,51]$} \\
\hline Punctanecine & Rindera umbellata & [169] \\
\hline Pycnanthine & Echium humile & [91] \\
\hline \multirow[t]{22}{*}{ Retronecine } & Anchusa hispida (Gastrocotyle hispida) & {$[40]$} \\
\hline & Echium vulgare & [96] \\
\hline & Heliotropium angiospermum & [106] \\
\hline & Heliotropium confertifolum & [106] \\
\hline & Heliotropium curassavicum & {$[106,116]$} \\
\hline & Heliotropium foliosissimum & [106] \\
\hline & Heliotropium fruticosum & [106] \\
\hline & Heliotropium gregii & [106] \\
\hline & Heliotropium indicum & {$[106,116]$} \\
\hline & Heliotropium keralense & {$[133]$} \\
\hline & Heliotropium molle & [106] \\
\hline & Heliotropium ovalifolium & [136] \\
\hline & Heliotropium procumbens & [106] \\
\hline & Heliotropium queretaroanum & [106] \\
\hline & Heliotropium racemosum & [106] \\
\hline & Heliotropium scabrum & [139] \\
\hline & Heliotropium spathulatum & {$[106,116]$} \\
\hline & Heliotropium sessei & [106] \\
\hline & Heliotropium ternatum & [106] \\
\hline & Heliotropium wigginsii & [106] \\
\hline & Lappula spinocarpos & [40] \\
\hline & Trichodesma africanum & {$[40]$} \\
\hline Retronecine-7:9- dibenzoate & Caccinea glauca & {$[58]$} \\
\hline $\begin{array}{l}\text { Retronecine 2S-hydroxy-2S(1S-hydroxyethyl)-4- } \\
\text { methyl-pentanosyl ester }\end{array}$ & Anchusa strigosa & {$[50,51]$} \\
\hline $\begin{array}{l}\text { Retronecine 2S-hydroxy-2S(1R-hydroxyethyl)-4- } \\
\text { methyl-pentanosyl ester }\end{array}$ & Anchusa strigosa & {$[50,51]$} \\
\hline \multirow[t]{10}{*}{ Rinderine } & Anchusa milleri & [40] \\
\hline & Arnebia decumbens & [52] \\
\hline & Cynoglossum columnae & [69] \\
\hline & Cynoglossum creticum & {$[70,72]$} \\
\hline & Cynoglossum officinale & {$[67,84]$} \\
\hline & Heliotropium indicum & {$[130,131]$} \\
\hline & Heliotropium peruvianum & [29] \\
\hline & Heliotropium transalpinum & [144] \\
\hline & Paracaryum intermedium & {$[40]$} \\
\hline & Paracaryum regulosum & [40] \\
\hline
\end{tabular}


Table 2. Cont.

\begin{tabular}{|c|c|c|}
\hline Compounds & Sources & References \\
\hline \multirow[t]{3}{*}{ Rinderine } & Rindera austroechinata & {$[57]$} \\
\hline & Rindera baldschuanica & [152] \\
\hline & Solanthus turkestanicus & {$[150]$} \\
\hline Scorpioidine & Myosotis scorpioides & [159] \\
\hline \multirow[t]{2}{*}{ 7-Senecioylhelotridine } & Cynoglossum creticum & {$[70]$} \\
\hline & Paracarum intermedium & {$[40]$} \\
\hline \multirow[t]{3}{*}{ 7-Senecioylretronecine } & Alkanna orientalis & [40] \\
\hline & Echium hypertropicum & [92] \\
\hline & Echium humile & [91] \\
\hline \multirow[t]{2}{*}{ 9-Senecioylretronecine } & Alkanna orientalis & [40] \\
\hline & Echium humile & [91] \\
\hline 7-Senecioylrinderine & Paracaryum intermedium & [40] \\
\hline 7-Senecioyllycopsamine & Echium humile & [91] \\
\hline Sincamidine & Amsinckia intermedia & {$[44]$} \\
\hline Senkirkine & Trichodesma ehrenbergii & [54] \\
\hline Strigosine & Heliotopium strigosum & {$[25,141]$} \\
\hline \multirow[t]{5}{*}{ Subulacine } & Heliotopium angiospermum & [29] \\
\hline & Heliotopium molle & [29] \\
\hline & Heliotopium subulacatum & [29] \\
\hline & Heliotopium ternatum & {$[27]$} \\
\hline & $\begin{array}{l}\text { Heliotopium transalpinum var. } \\
\text { transalpinum }\end{array}$ & [145] \\
\hline \multirow[t]{21}{*}{ Supinine } & Amsinckia carinata & [42] \\
\hline & Amsinckia eastwoodiae & {$[42]$} \\
\hline & Amsinckia furacata & [42] \\
\hline & Amsinckia lunaris & [42] \\
\hline & Amsinckia lycopsoides & {$[42,44]$} \\
\hline & $\begin{array}{l}\text { Amsinckia lycopsoides } \times \text { menziesii } \\
\text { var. } \text { intermedia }\end{array}$ & {$[42]$} \\
\hline & Amsinckia menziesii var. intermedia & [42] \\
\hline & Amsinckia retrosa & [42] \\
\hline & Amsinckia spectabilis var. microcarpa & [42] \\
\hline & Amsinckia spectabilis var. spectabilis & {$[42]$} \\
\hline & Amsinckia spectabilis var. nicolai & [42] \\
\hline & Amsinckia tessellate var. gloriosa & [42] \\
\hline & Amsinckia tessellate var. tessellate & [42] \\
\hline & Anchusa arvensis (Lycopsis arvensis) & {$[40]$} \\
\hline & Anchusa melleri & [40] \\
\hline & Arnebia decumbens & {$[52]$} \\
\hline & Borago officinalis & {$[55]$} \\
\hline & Caccina crassifolia & [57] \\
\hline & Cerinthe glabra & [59] \\
\hline & Cynoglossum amabile & {$[67]$} \\
\hline & Cynoglossum creticum & {$[70]$} \\
\hline
\end{tabular}


Table 2. Cont.

\begin{tabular}{|c|c|c|}
\hline Compounds & Sources & References \\
\hline \multirow[t]{10}{*}{ Supinine } & Heliotropium bacciferum & [109] \\
\hline & Heliotropium europaeum & [127] \\
\hline & Heliotropium hirsutissimum & [129] \\
\hline & Heliotropium indicum & {$[130,131]$} \\
\hline & Heliotropium supinum & {$[27,143]$} \\
\hline & Heliotropium transalpinum & [144] \\
\hline & Lappula spinocarpos & [40] \\
\hline & Tournefortia samentosa & {$[28]$} \\
\hline & Trichodesma ehrenbergii & [54] \\
\hline & Trichodesma zeylanicum & [182] \\
\hline \multirow[t]{14}{*}{ Supinidine } & Heliotropium angiospermum & [106] \\
\hline & Heliotropium confertifolum & [106] \\
\hline & Heliotropium curassavicum & {$[106,116]$} \\
\hline & Heliotropium foliosissimum & [106] \\
\hline & Heliotropium fruticosum & [106] \\
\hline & Heliotropium gregii & [106] \\
\hline & Heliotropium indicum & {$[106,116]$} \\
\hline & Heliotropium molle & [106] \\
\hline & Heliotropium procumbens & [106] \\
\hline & Heliotropium queretaroanum & [106] \\
\hline & Heliotropium racemosum & [106] \\
\hline & Heliotropium spathulatum & {$[106,116]$} \\
\hline & Heliotropium sessei & [106] \\
\hline & Heliotropium wigginsii & [106] \\
\hline $\begin{array}{l}\text { Supinidine N-oxide } 2 \mathrm{~S} \text {-hydroxy-2S(1S- } \\
\text { hydroxyethyl)-4-methyl-pentanoyl ester }\end{array}$ & Anchusa strigosa & {$[50,51]$} \\
\hline \multirow[t]{3}{*}{ Symlandine } & Amsinckia menziesii var. inermedia & [42] \\
\hline & Echium sericeum & [54] \\
\hline & Symphytum $\times$ uplandicum & [180] \\
\hline \multirow[t]{12}{*}{ Symphytine } & Myosotis scorpioides & [159] \\
\hline & Symphytum asperum & {$[171]$} \\
\hline & Symphytum bohemium & [173] \\
\hline & Symphytum consolidum & [175] \\
\hline & Symphytum grandiflorum & {$[176]$} \\
\hline & Symphytum ibericum & {$[176]$} \\
\hline & Symphytum officinale & {$[171,177-179]$} \\
\hline & Symphytum orientale & {$[28]$} \\
\hline & Symphytum peregrinum & [27] \\
\hline & Symphytum tanaiense & [173] \\
\hline & Symphytum tuberosum & {$[28,176]$} \\
\hline & Symphytum $\times$ uplandicum & {$[180]$} \\
\hline \multirow[t]{3}{*}{ Symviridine } & Symphytum asperum & {$[172]$} \\
\hline & Symphytum officinale & {$[172,177]$} \\
\hline & Symphytum $\times$ uplandicum & {$[172]$} \\
\hline
\end{tabular}


Table 2. Cont.

\begin{tabular}{|c|c|c|}
\hline Compounds & Sources & References \\
\hline \multirow[t]{15}{*}{ Tessellatine } & Amsinckia douglasiana & {$[42]$} \\
\hline & Amsinckia eastwoodiae & {$[42]$} \\
\hline & Amsinckia furacata & {$[42]$} \\
\hline & Amsinckia grandiflora & {$[42]$} \\
\hline & Amsinckia lunaris & [42] \\
\hline & Amsinckia lycopsoides & {$[42,44]$} \\
\hline & $\begin{array}{l}\text { Amsinckia lycopsoides } \times \text { menziesii } \\
\text { var. intermedia }\end{array}$ & {$[42]$} \\
\hline & Amsinckia menziesii var. intermedia & [42] \\
\hline & Amsinckia retrosa & {$[42]$} \\
\hline & Amsinckia spectabilis var. microcarpa & [42] \\
\hline & Amsinckia spectabilis var. nicolai & {$[42]$} \\
\hline & Amsinckia tessellate var. gloriosa & [42] \\
\hline & Amsinckia tessellate var. tessellate & [42] \\
\hline & Cryptantha confertiflora & [63] \\
\hline & Cryptantha virginiensis & [63] \\
\hline Thesinine & Borago officinalis & {$[56]$} \\
\hline Thesinine-4'- $O-\beta$-D-glucoside & Borago officinalis & {$[56]$} \\
\hline $\begin{array}{l}\text { 7-Tigloyl-9-(2-deoxy-2- } \\
\text { methyl)echimidinylheliotridinEchium }\end{array}$ & Cynoglossum columnae & [69] \\
\hline \multirow[t]{4}{*}{ 7-Tigloyl-9-(2,3-dihydroxybutyryl)retronecine } & Echium horridum & {$[90]$} \\
\hline & Echium rauwolfii & {$[90]$} \\
\hline & Echium setosum & [96] \\
\hline & Echium vulgare & {$[96]$} \\
\hline \multirow[t]{2}{*}{ 7-Tigloyl-9-(2,3-dihydroxypropanoyl)retronecine } & Alkanna orientalis & {$[40]$} \\
\hline & Alkanna tincotoria & {$[40]$} \\
\hline 7-Tigloylheliotridine & Cynoglossum officinale & [67] \\
\hline \multirow[t]{2}{*}{ 7-Tigloyllycopsamine } & Echium horridum & {$[90]$} \\
\hline & Echium rauwolfii & [90] \\
\hline \multirow[t]{4}{*}{ 7-Tigloyl-9-(2-methybutyryl)retronecine } & Echium horridum & [90] \\
\hline & Echium rauwolfii & [90] \\
\hline & Echium setosum & [96] \\
\hline & Echium vulgare & [96] \\
\hline \multirow[t]{5}{*}{ 7-Tigloylretronecine } & Alkanna tinctoria & [40] \\
\hline & Arnebia decumbens & [40] \\
\hline & Echium amoenum & [87] \\
\hline & Echium setosum & [96] \\
\hline & Echium vulgare & [96] \\
\hline \multirow[t]{7}{*}{ 9-Tigloylretronecine } & Alkanna orientalis & {$[40]$} \\
\hline & Alkanna tincotoria & {$[40]$} \\
\hline & Arnebia decumbens & {$[52]$} \\
\hline & Echium horridum & {$[90]$} \\
\hline & Echium rauwolfii & {$[90]$} \\
\hline & Echium setosum & {$[96]$} \\
\hline & Echium vulgare & {$[96]$} \\
\hline
\end{tabular}


Table 2. Cont.

\begin{tabular}{|c|c|c|}
\hline Compounds & Sources & References \\
\hline \multirow{15}{*}{ Trachelanthamidine } & Heliotropium angiospermum & [106] \\
\hline & Heliotropium confertifolum & {$[106]$} \\
\hline & Heliotropium curassavicum & {$[106,116,118]$} \\
\hline & Heliotropium foliosissimum & {$[106]$} \\
\hline & Heliotropium fruticosum & {$[106]$} \\
\hline & Heliotropium gregii & {$[106]$} \\
\hline & Heliotropium indicum & {$[106,116]$} \\
\hline & Heliotropium molle & {$[106]$} \\
\hline & Heliotropium procumbens & {$[106]$} \\
\hline & Heliotropium queretaroanum & {$[106]$} \\
\hline & Heliotropium racemosum & {$[106]$} \\
\hline & Heliotropium sessei & {$[106]$} \\
\hline & Heliotropium spathulatum & {$[106,116]$} \\
\hline & Heliotropium strigosa & {$[25]$} \\
\hline & Heliotropium wigginsii & {$[106]$} \\
\hline \multirow[t]{11}{*}{ Trachelanthamine } & Anchusa hispida & {$[40]$} \\
\hline & Cynoglossum clandestinum & [60] \\
\hline & Cynoglossum creticum & {$[70]$} \\
\hline & Cynoglossum officinale & [67] \\
\hline & Lappula spinocarpos & {$[40]$} \\
\hline & Myosotis sylvatica & {$[25]$} \\
\hline & Onosma stellulatum & {$[167]$} \\
\hline & Rindera balaschuanica & {$[152]$} \\
\hline & R. echinata & {$[120]$} \\
\hline & Trachelanthus hissaricus & [29] \\
\hline & Trachelanthus korolkovii & {$[57,150]$} \\
\hline \multirow[t]{2}{*}{ Trachelanthine } & Trachelanthus hissaricus & [29] \\
\hline & Trachelanthus korolkovii & {$[150]$} \\
\hline 7-Trachelanthyl-laburnine & Rindera umbellata & [169] \\
\hline 7-Trachelanthylretronecine & Amsinckia vernicosa & {$[42]$} \\
\hline Transalpinecine & $\begin{array}{l}\text { Heliotropium transalpinum var. } \\
\text { transalpinum }\end{array}$ & {$[145]$} \\
\hline \multirow[t]{2}{*}{ Triangularine } & Alkanna orientalis & {$[40]$} \\
\hline & Alkanna tinctoria & {$[40]$} \\
\hline \multirow[t]{2}{*}{ Triangularicine } & Alkanna orientalis & [40] \\
\hline & Alkanna tinctoria & {$[40]$} \\
\hline \multirow[t]{3}{*}{ Trichodesmine } & Heliotropium arguzioides & {$[28]$} \\
\hline & Trichodesma africanum & {$[40,181]$} \\
\hline & T. incanum & {$[46]$} \\
\hline \multirow[t]{3}{*}{ Turkestanine } & Rindera baldschuanica & {$[152]$} \\
\hline & Rindera oblongifolia & {$[152]$} \\
\hline & Solenanthus turkestanicus & {$[135,150]$} \\
\hline \multirow[t]{3}{*}{ Uplandicine } & Echium rauwolfii & [90] \\
\hline & Echium setosum & [96] \\
\hline & Echium vulgare & [96] \\
\hline
\end{tabular}


Table 2. Cont.

\begin{tabular}{|c|c|c|}
\hline Compounds & Sources & References \\
\hline \multirow[t]{3}{*}{ Uplandicine } & Onosma arenaria & {$[163]$} \\
\hline & Onosma stellulatum & [167] \\
\hline & Symphytum $\times$ uplandicum & {$[180]$} \\
\hline Uluganine & Ulugbekia tshimganica & [183] \\
\hline \multirow[t]{2}{*}{ Viridantine } & Cynoglossum furcatum & {$[76,187]$} \\
\hline & Onosma erecta & [69] \\
\hline \multirow[t]{14}{*}{ Viridiflorine } & Anchusa milleri & {$[40]$} \\
\hline & Cynoglossum germanicum & {$[77]$} \\
\hline & Cynoglossum officinale & {$[40,84]$} \\
\hline & Cynoglossum viridiforum & [97] \\
\hline & Lappula spinocarpus & {$[40]$} \\
\hline & Lindelofia olgae & [150] \\
\hline & Lindelofia pterocarpa & {$[151]$} \\
\hline & Lindelofia stylosa & {$[135]$} \\
\hline & Lindelofia tschimganic & [152] \\
\hline & Paracaryum intermedium & {$[40]$} \\
\hline & Paracaryum regulosum & {$[40]$} \\
\hline & Symphytum officinale & [179] \\
\hline & Trachelanthus hissricus & [29] \\
\hline & Trichodesma africanum & [40] \\
\hline \multirow[t]{5}{*}{ 7-Viridiflorylretronecine } & Echium tuberculatum & {$[60]$} \\
\hline & Eritrichium rupestre & {$[60]$} \\
\hline & Nonnea lutea & {$[60]$} \\
\hline & Nonnea setosa & {$[60]$} \\
\hline & Onosma stellulatum & {$[60]$} \\
\hline Vulgarine & Echium vulgare & [11] \\
\hline
\end{tabular}

\section{Phytochemical Analysis of PAs}

Various analytical techniques have been used for extraction, separation, identification and quantification of PAs. Recently, updated reviews were published [188,189] on the analysis of PAs in plants and foods along with different methods of preparation and extraction of PAs from different matrices including plants are their parts, such as seeds, pollen, but also from honey, body fluids, and insects. In addition to column chromatography and HPLC, droplet counter current chromatography (DCCC) has been used in preparative separation of pyrrolizidine alkaloids [190]. Advantages of this method include total sample recovery, good resolution and high reproducibility. A high-speed CCC was applied for preparative separation and purification of PAs from Amsinckia tessellata, Symphytum spp. and Trichodesma incanum [46]. For analytical purposes high-resolution capillary GLC alone or in combination with mass spectrometry is the method of choice for free PA bases [40,59,67,70,90,91,96,163,191-195]. HPLC and HPLC-MS are also helpful but less sensitive than GLC and GLC-MS [60,93,196-199]. In addition, the resolution is much lower than for GLC but in HPLC also PA-N-Oxides can be directly analysed. The present review describes the most common analytical tools for the analysis of PAs with an emphasis of mass spectrometry (mostly based on GLC-MS analyses). 


\subsection{Mass Spectrometry}

Mass spectrometry is an important and sensitive tool for the identification and structural determination of PAs. The advantages of mass spectrometry are high sensitivity and possibilities of combination with liquid chromatographic methods (e.g., GLC or HPLC) for the analysis of complex mixtures which usually exist in natural sources. Mass spectral data of PAs have been documented in several publications so that a comprehensive database exists. With respect to PAs, the mass spectra provide the molecular mass, type and structure of necine nucleus (saturation at 1, 2-position), sites of hydroxylation and acylation of the hydroxyl groups (as monoester and/or diester). However, MS alone does not provide all necessary structural information (especially, stereochemistry) necessary for an unambiguous assignment of the structure to an unknown compound. There is no difference between the mass spectra of the epimeric pairs e.g., the spectrum of 7-angeloylretronecine is identical to that of 7-angeloylheliotridine, the OH-groups at C-7 are $\beta$ and $\alpha$-oriented, respectively. The same situation exists with the stereoisomer pair echimidine and heliosupine. Echinatine, rinderine (heliotridine bases), lycopsamine, intermedine and indicine (retronecine bases) esterified at C-9 with the stereoisomeric acids, (-)-viridifloric, $(+)$-trachelanthic, $(-)$-viridifloric, $(+)$-trachelanthic and (-)-trachelanthic exhibit almost identical mass spectra (Figures 3-15).

Mass spectra of PAs provide important informations about the structure of necine substituents. The 1,2-unsaturated necine esterified at C-9 with a free hydroxyl group at C-7 exhibit a base peak at $\mathrm{m} / \mathrm{z} 138$, whereas acylation of C-7 (uplandicine, 7-acetyllycopsamine) provide a base peak at $\mathrm{m} / \mathrm{z} 180$. PAs with 1,2-unsaturated necines but without a hydroxyl group at C-7 (as in supinidine type) with an esterification at C-9 results in a base peak $m / z$ 122, while the saturated necine of the same type (trachelanthamidine, isoretroncanol, lindelofidine) shows a base peak at $\mathrm{m} / \mathrm{z}$ 124. 1,2-unsaturated diester PAs esterified with angelic, tiglic or senecioic at C-7 shows a base peak at $m / z 220$ [40,42,59,67,70,90,91,96,163,193,200,201]. The fragmentation pathway of this type of PAs starts with the cleavage of the weak allylic ester bond at C-9.

Figure 3. Mass spectrum of retronecine/heliotridin.

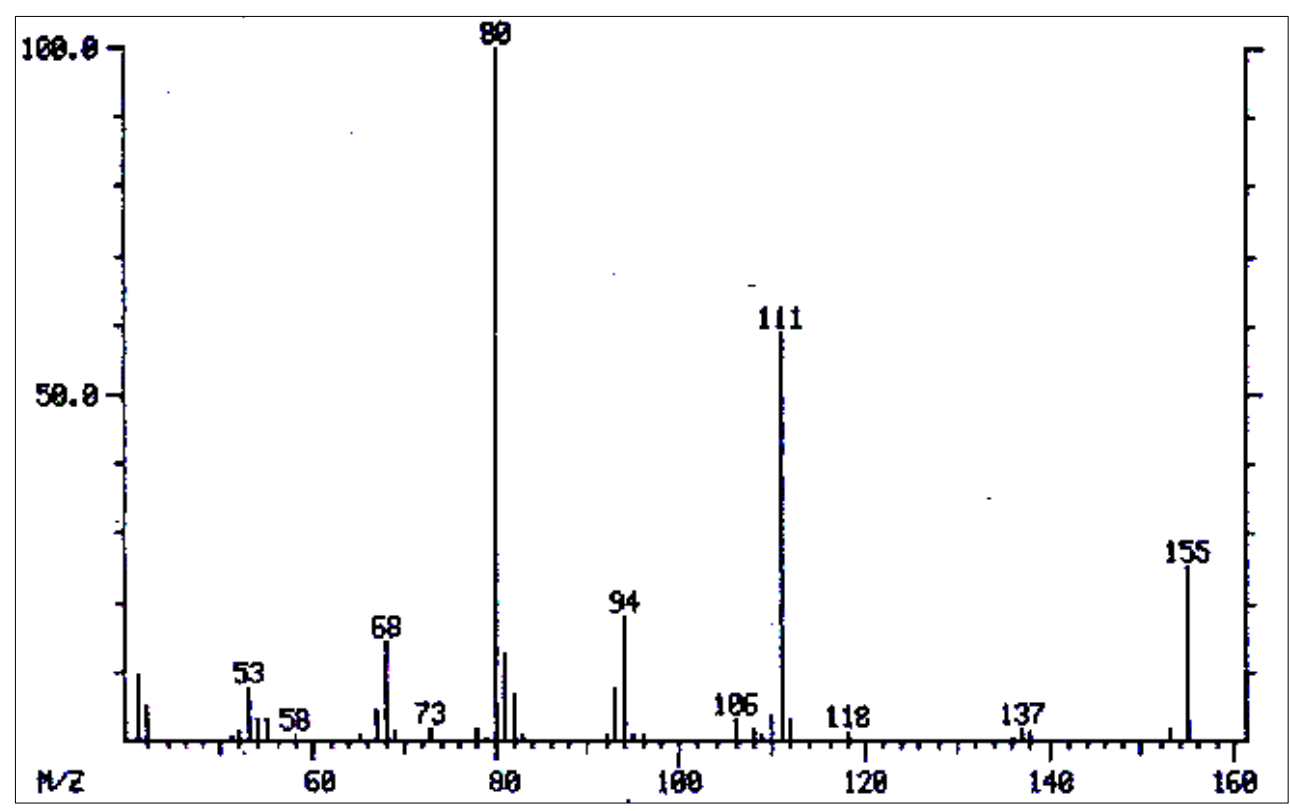


Figure 4. Mass spectrum of 9-acetyltrachelanthamidine.

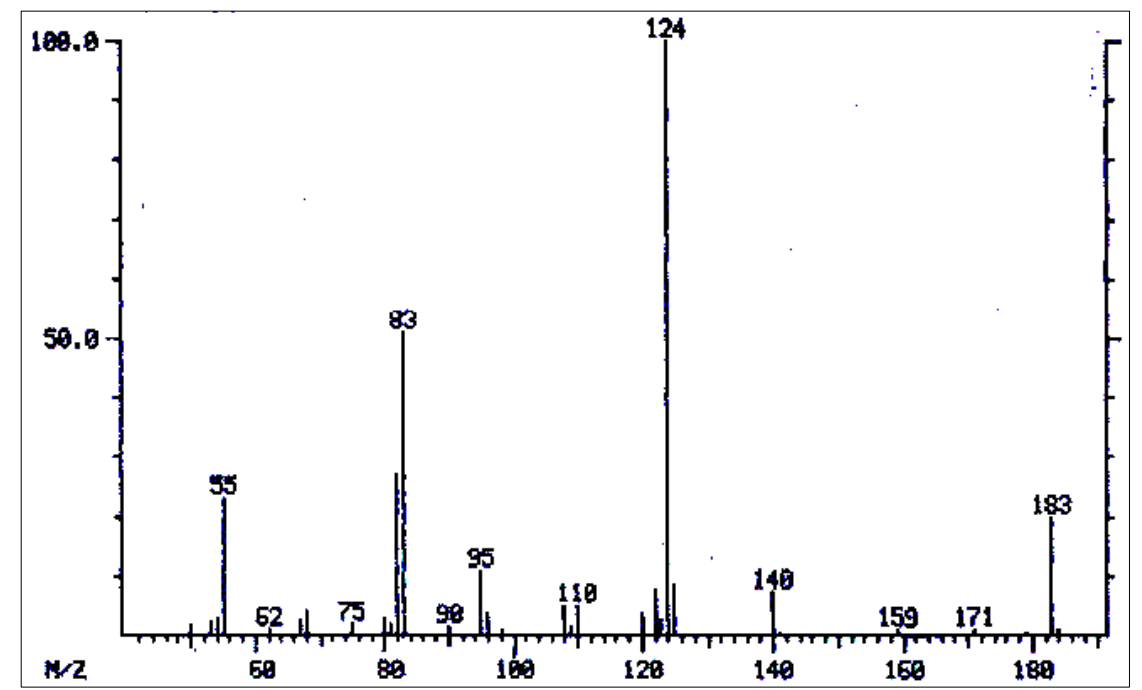

Figure 5. Mass spectrum of 7-acetylretronecine/7-acetylheliotridine.

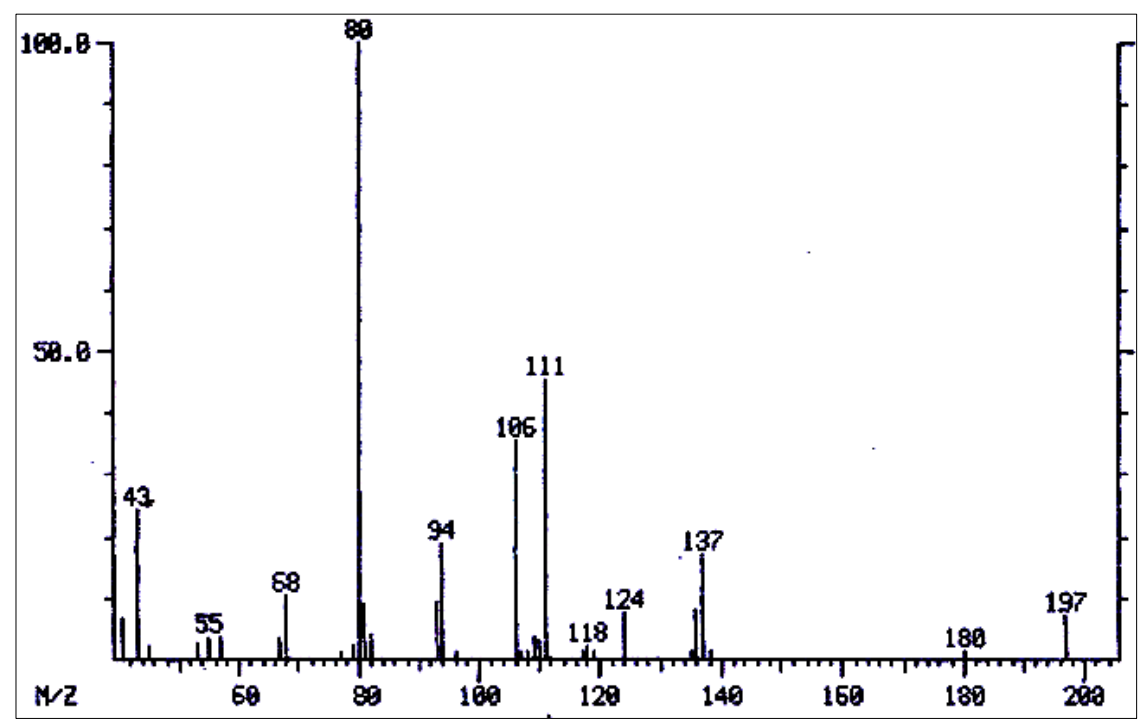

Figure 6. Mass spectrum of viridiflorine/trachelanthamine.

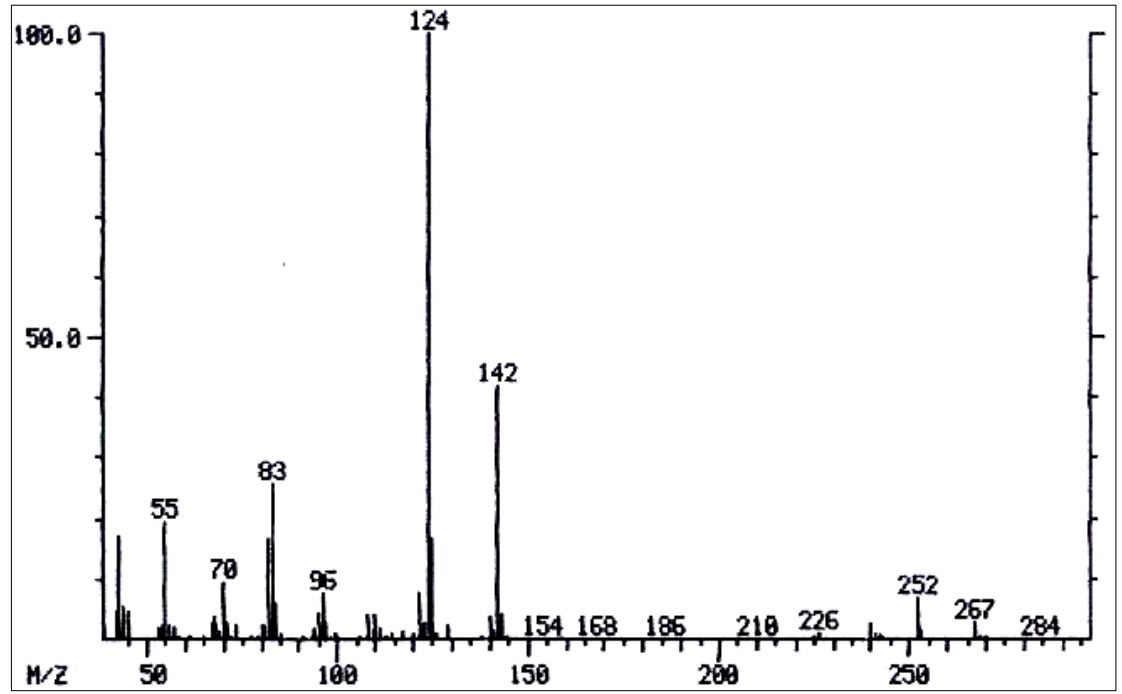


Figure 7. Mass spectrum of 9-angeloyl or 9-tigloylretronecine 9-angeloyl or 9-tigloylheliotridine.

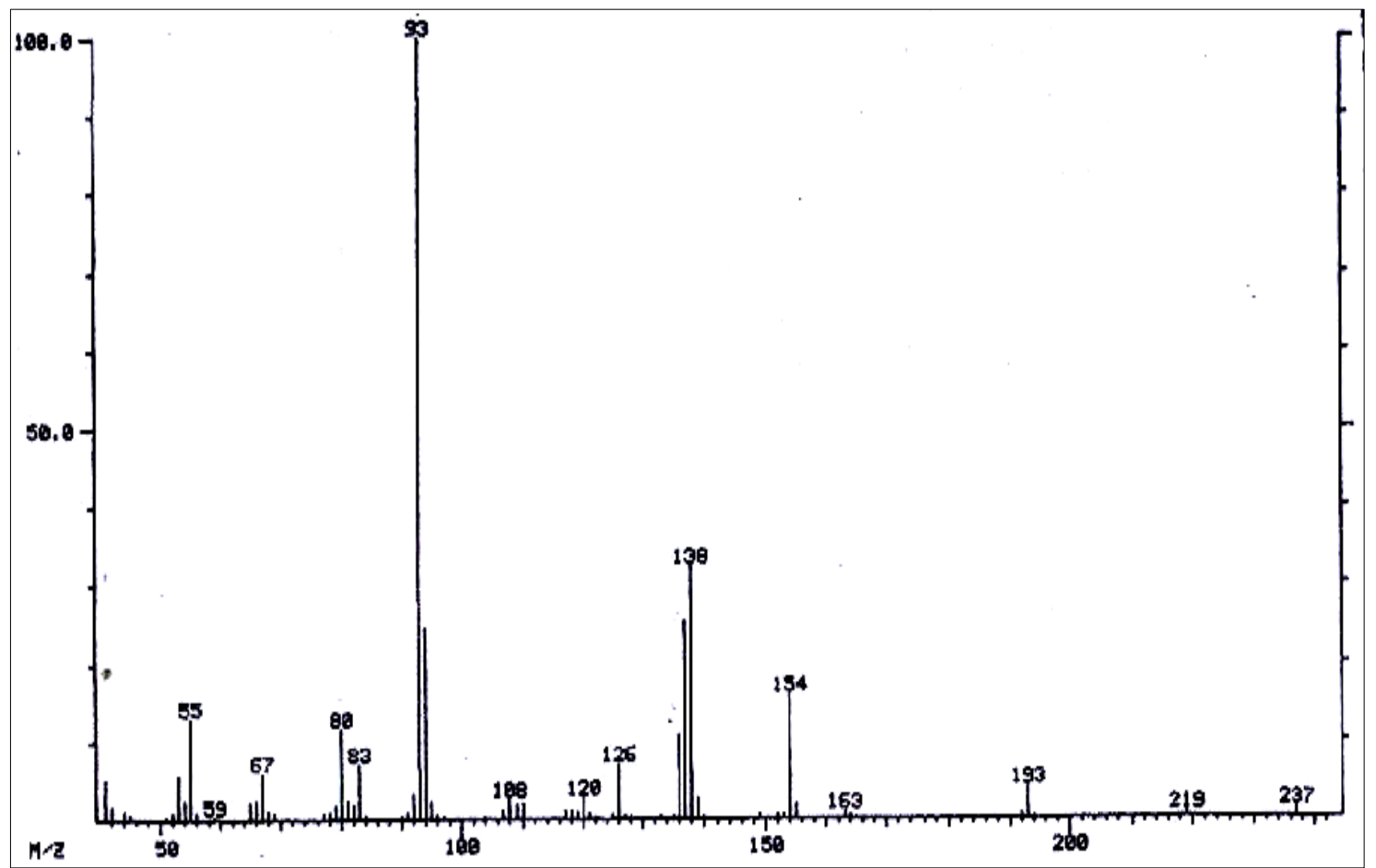

Figure 8. Mass spectrum of amabiline.

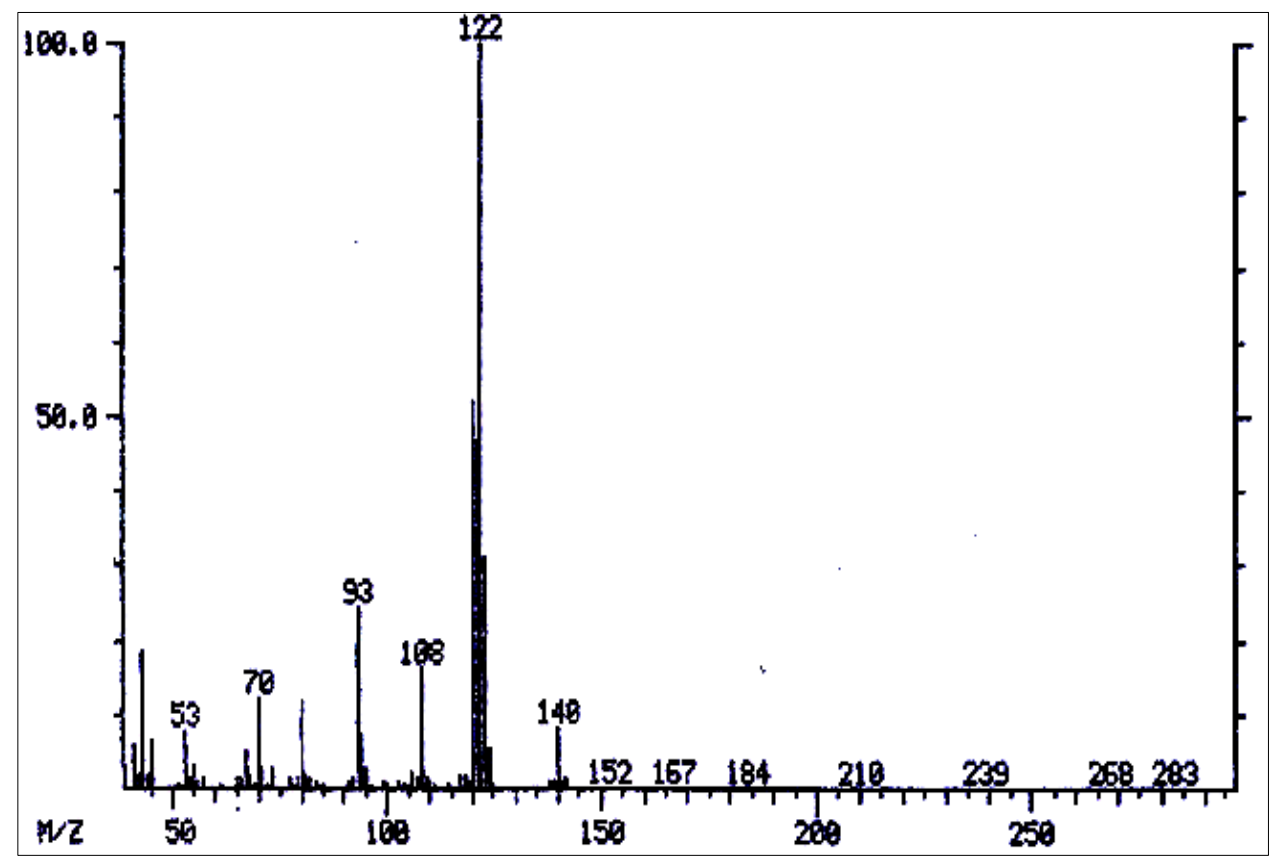


Figure 9. Mass spectrum of 9-curassavoylheliotridine.

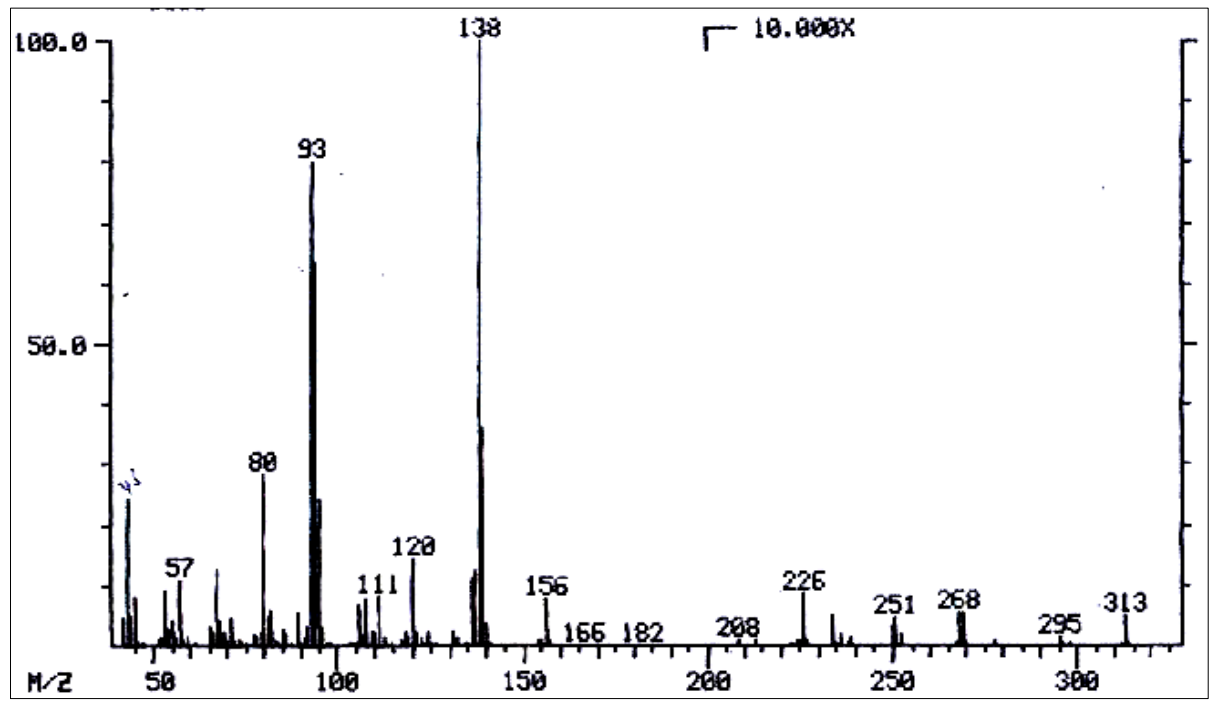

Figure 10. Mass spectrum of heliotrine.

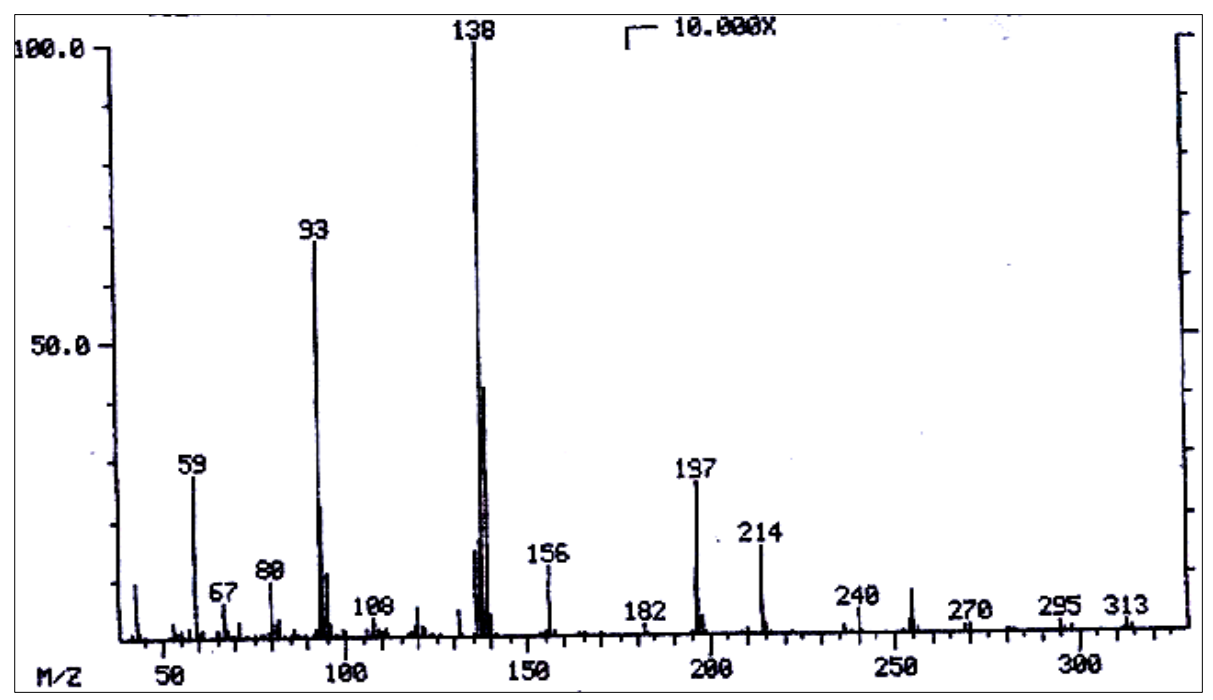

Figure 11. Mass spectrum of lycopsamine/intermedine/indicine/echinatine/rinderine.

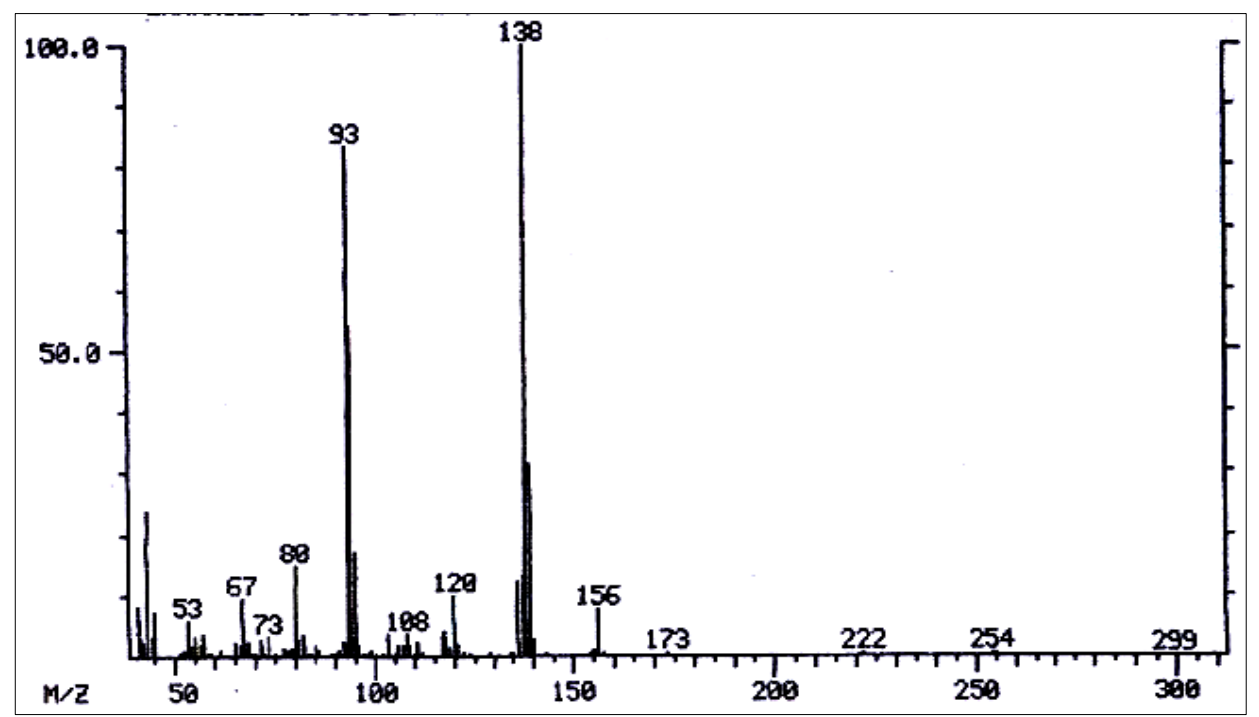


Figure 12. Mass spectrum of 7-acetyllycopsamine/7-acetyl intermedine/7-acetyl indicine/ 7-acetyl echinatine/7-acetyl rinderine.

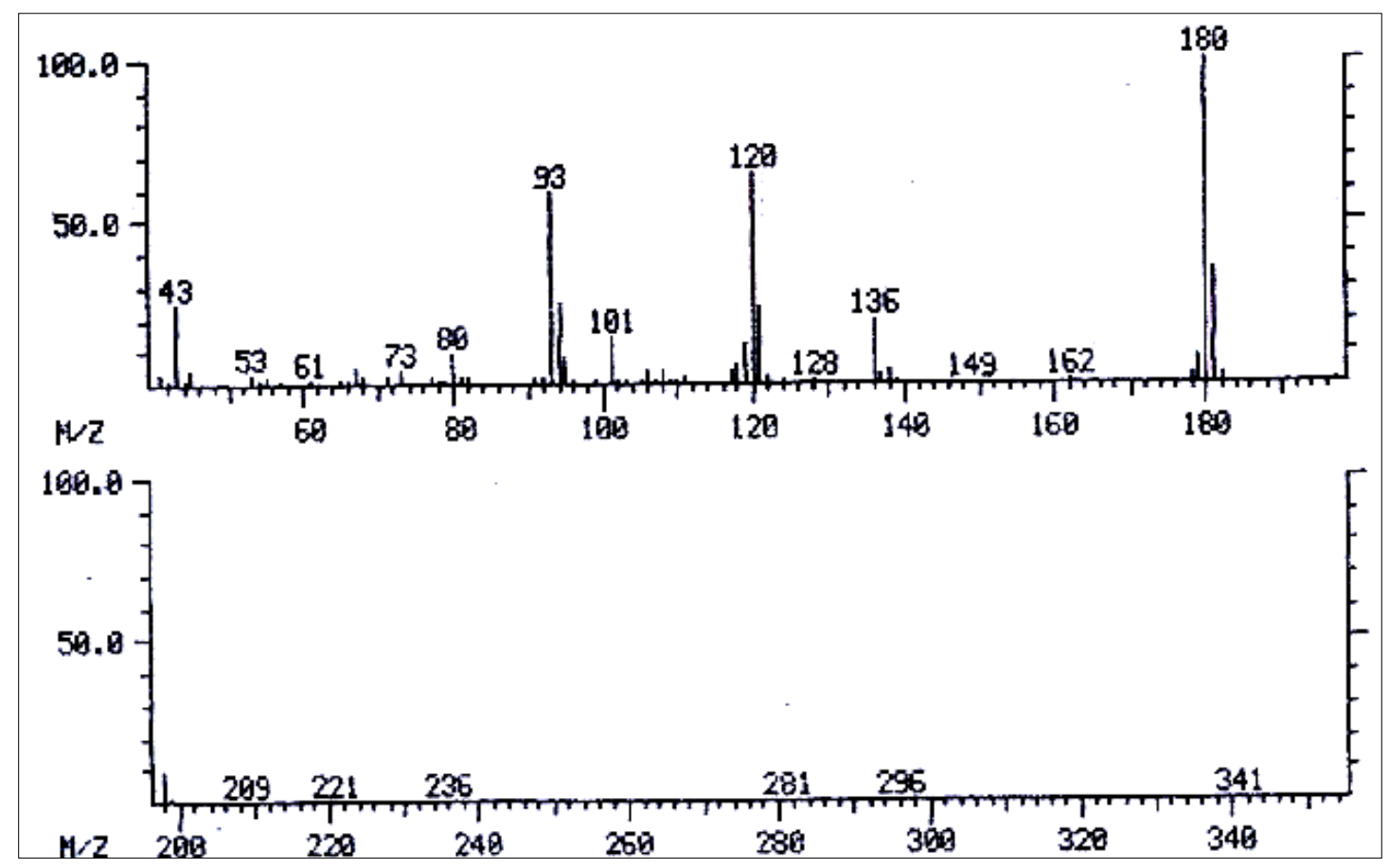

Figure 13. Mass spectrum of 3',7-diacetyllycopsamine/3',7-diacetyl intermedine/ 3',7-diacetyl indicine/3',7-diacetyl echinatine/3',7-diacetyl rinderine.

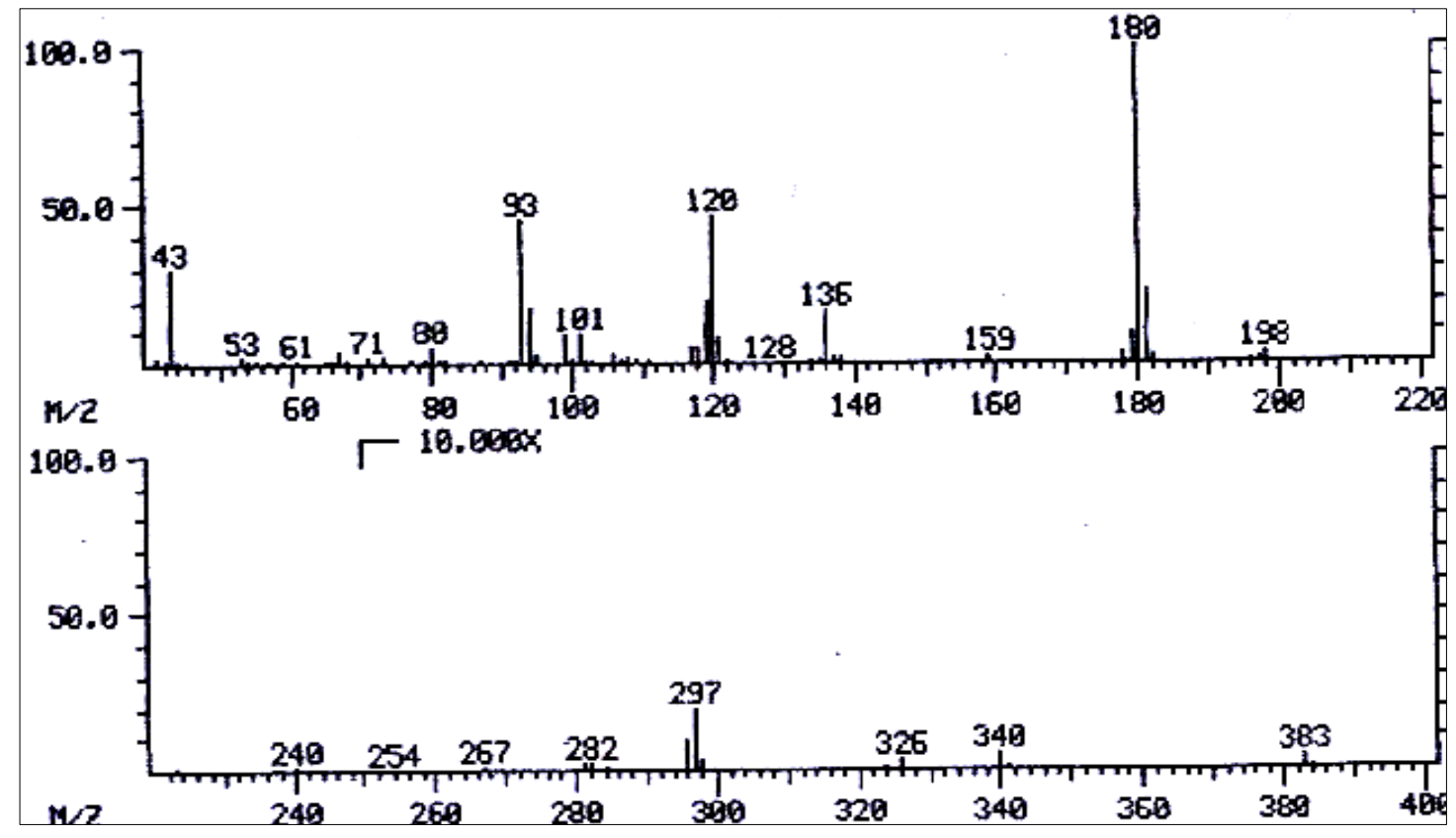


Figure 14. Mass spectrum of echimidine/heliosupine.

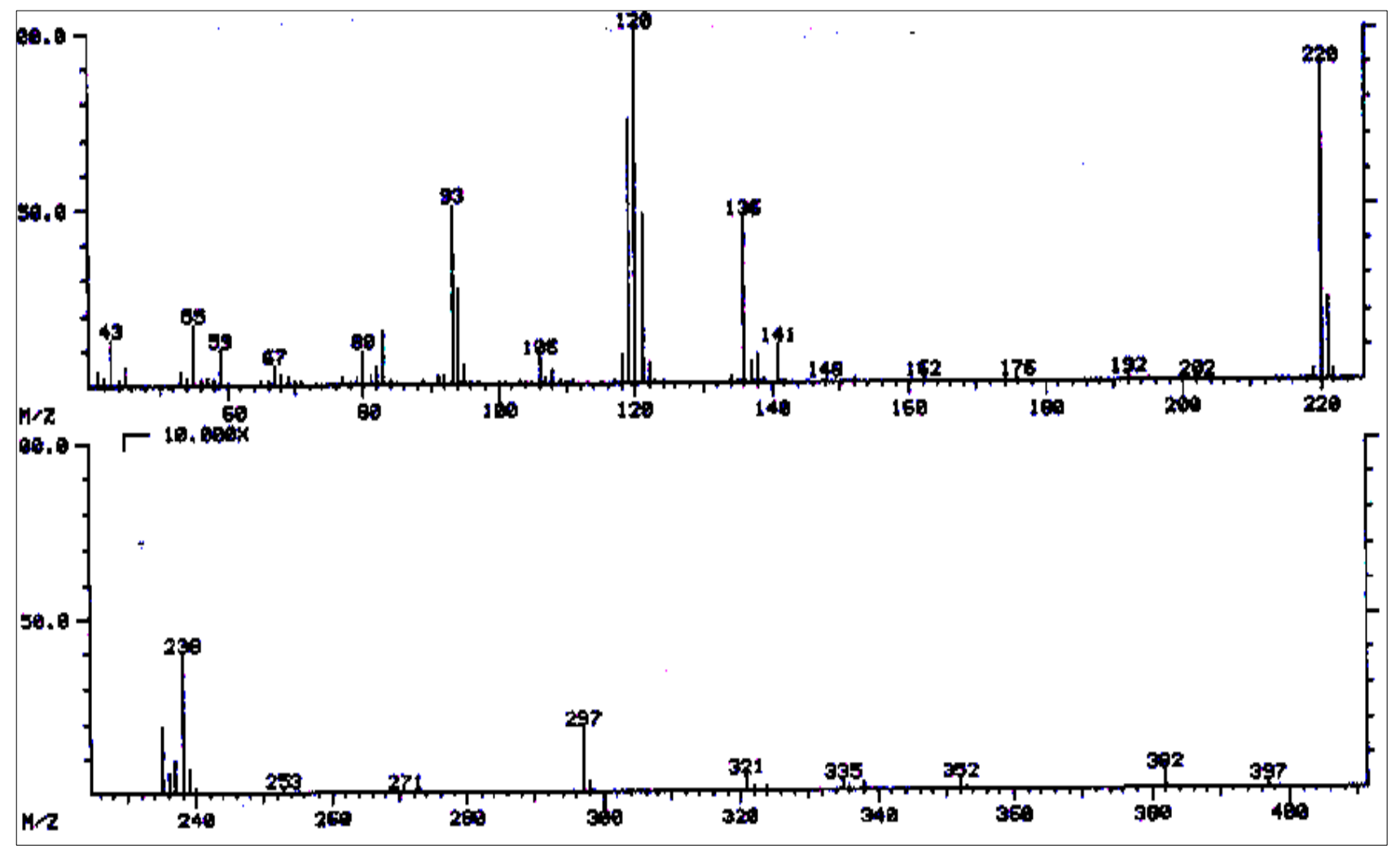

Figure 15. Mass spectrum of 3 '-acetylechimidine/3'-acetylheliosupine.

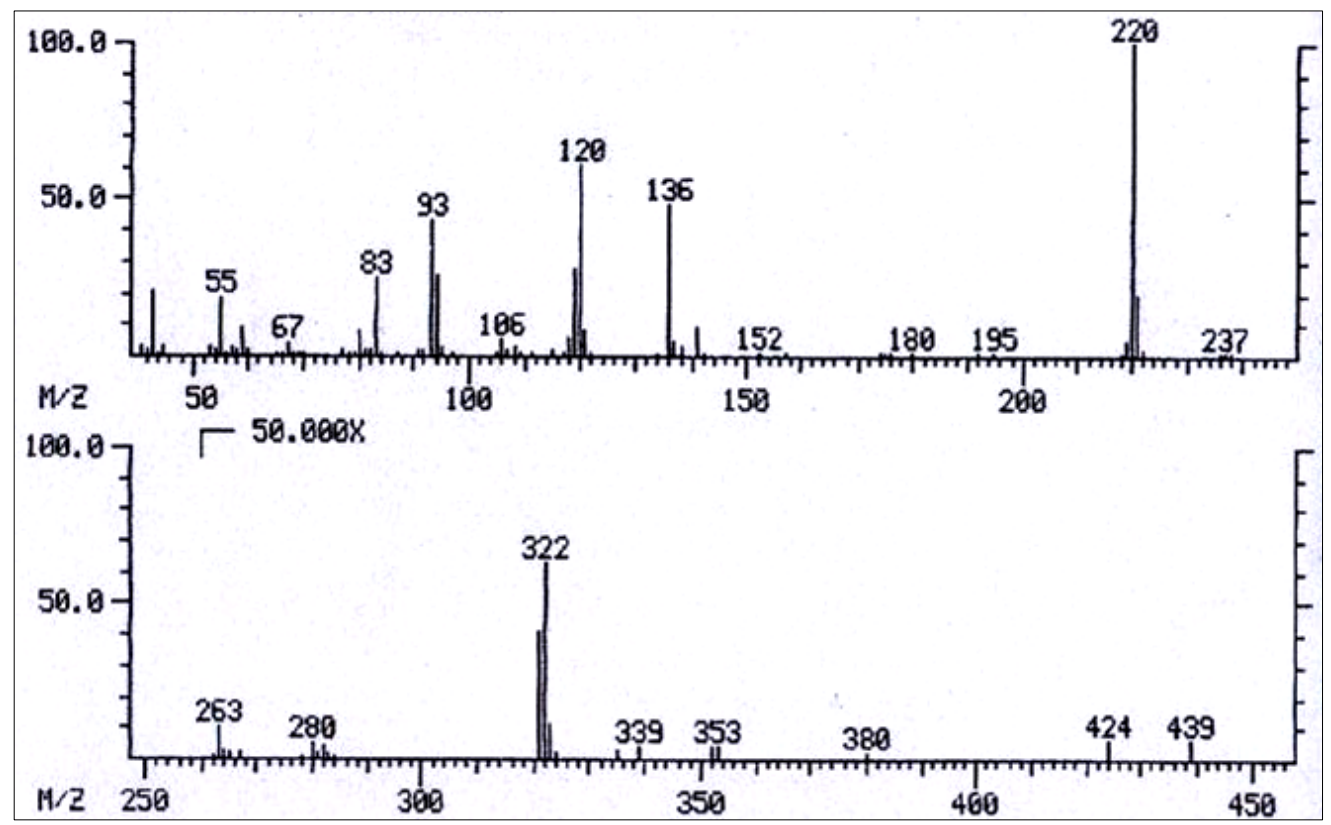

In our review we have tabulated the MS data useful for the identification and structural studies of PAs in Boraginaceae; Table 3 summarizes the mass spectral data (GLC-MS, LC-MS, direct inlet) of corresponding PAs. 
Table 3. Mass data of PAs (mostly derived from gas-liquid chromatography-mass spectrometry (GLC-MS) analyses) for all compounds identified in the Boraginaceae. Compounds are numbered as in Figure 16.

\begin{tabular}{|c|c|c|c|c|c|}
\hline No. & Alkaloid & RI & {$[\mathbf{M}]^{+}$} & Characteristic ions $m / z$ (relative abundance) & References \\
\hline 1 & 3'-Acetylcanescine & - & 441 & $\begin{array}{l}\text { 441(0.7), 426(2.6), 355(2.4), 255(6.3), 238(62.4), 220(18.3), 180(39), 136(47), 120(100), 93(74), } \\
80(20) .\end{array}$ & [154] \\
\hline 2 & 3'- Acetylcanescenine & - & 441 & $\begin{array}{l}441(1.2), 426(2.4), 398(1.6), 355(1.2), 255(5.7), 238(58.2), 220(20.3), 180(44.3), 136(43.4), \\
120(100), 93(80.3), 80(22.1) .\end{array}$ & [154] \\
\hline 3 & $\begin{array}{l}\text { 7-Acetyl-9- } \\
\text { curassavoylheliotridine }\end{array}$ & 2275 & 355 & $\begin{array}{l}\text { 295(0.1), 268(0.2), 198(8), 181(36), 180(100), 136(22), 121(26), 120(84), 119(12), 95(15), } \\
93(35), 80(17), 67(10), 57(12), 45(9), 43(48) .\end{array}$ & [40] \\
\hline 4 & $\begin{array}{l}\text { 7-Acetyl-9-(2,3-dihydroxybutryl) } \\
\text { retronecine }\end{array}$ & 2092 & 299 & $\begin{array}{l}\text { 239(3), 181(20), 180(100), 136(20), 120(59), 101(20), 94(32), 93(73), 80(12), 67(5), 55(5), } \\
43(15) .\end{array}$ & [163] \\
\hline 5 & $\begin{array}{l}\text { 7-Acetyl-9-(2,3-dimethylbutryl) } \\
\text { retronecine }\end{array}$ & $1947^{* *}$ & 295 & 235(8), 180(100), 136(20), 120(36), 101(30), 94(30), 93(65), 80(8), 43(42). & [163] \\
\hline 6 & 3'-Acetylechinatine & 2220 & 341 & $\begin{array}{l}326(0.1), 298(1), 255(2), 254(2), 181(2), 156(4), 139(21), 138(100), 137(10), 136(10), 120(6), \\
99(6), 94(20), 93(71), 80(8), 67(5), 43(22) .\end{array}$ & [84] \\
\hline 7 & 7-Acetylechinatine & 2235 & 341 & $\begin{array}{l}341(0.1), 281(2), 198(6), 181(39), 180(100), 136(18), 121(35), 120(70), 119(28), 101(9), 94(18), \\
93(55), 80(7), 43(33) .\end{array}$ & [67] \\
\hline 8 & 3'-Acetylechiumine & $2245^{*}$ & 423 & $\begin{array}{l}\text { 423(2), 380(3), 338(3), 337(6), 336(3), 323(2), 280(3), 263(3), 256(2), 238(5), 237(6), 221(34), } \\
220(100), 219(15), 159(5), 141(19), 138(12), 136(54), 121(20), 120(76), 119(34), 106(12), 94(42), \\
93(56), 83(27), 80(15), 59(3), 55(34), 53(17) .\end{array}$ & [42] \\
\hline 9 & 3'-Acetylechimidine & 2640 & 439 & $\begin{array}{l}\text { 439(0.1), 424(0.1), 322(1), 238(2), 221(25), 220(100), 219(3), 138(5), 137(6), 136(49), 121(8), } \\
120(61), 119(28), 106(6(, 94(24), 93(46), 83(14), 59(11), 55(13) .\end{array}$ & [91] \\
\hline 10 & 5'-Acetyleuropine & - & 371 & 156(18), 138(100), 94(36), 93(75), 59(74). & [138] \\
\hline 11 & 7-Acetyleuropine & - & 371 & 371(2.7), 356(1), 311(3), 282(5), 180(100), 120(72), 93(65), 80(17), 59(95). & [110] \\
\hline 12 & 3'-Acetylfurcatine & $3188^{*}$ & 425 & 223(28), 222(100), 143(10), 136(36), 121(15), 120(82), 94(27), 93(61), 85(21), 80(7), 57(63). & [42] \\
\hline 13 & 3'-Acetylheliosupine & 2640 & 439 & $\begin{array}{l}424(0.1), 321(1), 221(28), 220(100), 141(10), 138(4), 137(5), 136(45), 121(14), 120(89), 119(82), \\
106(7), 94(21), 93(40), 83(11), 80(5), 59(8), 55(11), 43(22) .\end{array}$ & [67] \\
\hline 14 & $\begin{array}{l}\text { 7-Acetyl-9-(2-hydroxy-3- } \\
\text { methylbutryl) retronecine }\end{array}$ & $2024^{* *}$ & 297 & 237(5), 180(100), 136(25), 120(52), $119(20), 101(25), 94(45), 93(95), 80(15), 43(35)$. & [163] \\
\hline 15 & 3'-Acetylindicine & 2195 & 341 & $341(2), 255(2), 181(5), 138(100), 93(48), 43(10)$. & [158] \\
\hline
\end{tabular}


Table 3. Cont

\begin{tabular}{|c|c|c|c|c|c|}
\hline No. & Alkaloid & RI & {$[\mathbf{M}]^{+}$} & Characteristic ions $m / z$ (relative abundance) & References \\
\hline 16 & 3'-Acetylintermedine & 2255 & 341 & $\begin{array}{l}\text { 341(5), } 298 \text { (4), } 255 \text { (16), } 139 \text { (20),138(100), } 137 \text { (12),136 (12), } 94 \text { (30), } 93 \text { (71), } 80 \text { (10), } 43 \\
(21) .\end{array}$ & [40] \\
\hline 17 & 7-Acetylintermedine & 2220 & 341 & $\begin{array}{l}\text { 296(4), 281(3), 181(40), 180(100), 136(25), 121(30), 120(78), 119(15), 101(19), 95(10), 94(30), } \\
\text { 93(73), 80(11), 43(40). }\end{array}$ & [40] \\
\hline 18 & 5'-Acetyllasiocarpine & - & 453 & $363,335,321,263,220(100), 141,136,121,120,119,106,94,93,83,80$ & [186] \\
\hline 19 & 3'-Acetyllithosenine & - & 457 & $\begin{array}{l}\text { 457(0.78), 442(2), 339(1), 255(2), 238(100), 237(5), 222(10), 220(25), 138(30), 137(8), 136(49), } \\
121(36), 120(75), 119(36), 95(9), 94(34), 93(57), 80(12) .\end{array}$ & [156] \\
\hline 20 & 3'-Acetyllycopsamine & 2255 & 341 & 341(5), 298(4), 255(16), 139(20), 138(100), 137(12), 136(12), $94(30), 93(71), 80(10), 43(21)$. & [40] \\
\hline 21 & 7-Acetyllycopsamine & 2230 & 341 & $\begin{array}{l}341(1), 296(4), 281(3), 181(32), 180(100), 136(20), 121(35), 120(70), 119(11), 101(19), 95(10), \\
94(28), 93(61), 80(10), 43(36) .\end{array}$ & [91] \\
\hline 22 & $\begin{array}{l}\text { 7-Acetyl-9-(2-methylbutyryl) } \\
\text { retronecine }\end{array}$ & $1914^{* *}$ & 281 & $\begin{array}{l}\text { 221(12), 195(5), 180(100), 136(25), 120(42), 119(15), 101(35), 94(45), 93(80), 80(13), 67(100), } \\
57(18), 53(5), 43(23) \text {. }\end{array}$ & [163] \\
\hline 23 & 3'-Acetylmyscorpine & $3290^{*}$ & 423 & 221(30), 220(100), 136(47), 120(67), 119(35), 94(39), 93(51), 83(32), 80(19), 55(39). & [42] \\
\hline 24 & 7-Acetylretronecine & $1532 * *$ & 197 & 180(3), 137(12), 111(40), 106(30), 94(20), 80(100), 68(3), 43(30). & [163] \\
\hline 25 & 3'-Acetylrinderine & 2222 & 341 & $\begin{array}{l}326(0.1), 298(1), 255(1), 254(0.5), 181(5), 156(5), 139(30), 138(100), 137(17), 136(17), 120(10) \text {, } \\
99(10), 95(9), 94(35), 93(95), 80(13), 67(9), 43(36) .\end{array}$ & [70] \\
\hline 26 & $\begin{array}{l}\text { 7-Acetyl-9-(sarracinoyl) } \\
\text { retronecine }\end{array}$ & 2125 & 295 & $\begin{array}{l}\text { 235(1), 197(32), 196(10), 181(8), 180(55), 179(15), 136(34), 121(12), 120(42), } 119(20), \\
101(19), 94(40), 93100), 80(15), 67(6), 53(9), 43(20) .\end{array}$ & [40] \\
\hline 27 & 7-Acetylscorpioidine & - & 423 & $\begin{array}{l}\text { 423(0.8), 181(23), 180(92), 179(17), 136(23), 121(10), 120(57), 119(45), 95(6), 94(35), 93(57), } \\
83(100), 80(15), 55(39), 43(30) .\end{array}$ & [159] \\
\hline 28 & 3'-Acetylsupinine & $2080^{* *}$ & 325 & $\begin{array}{l}\text { 284(.02), 299(10), 136(10), 122(100), 120(48), 108(13), 101(13), 93(50), 80(13), 70(9), 53(6), } \\
\text { 43(15). }\end{array}$ & [163] \\
\hline 29 & 3'-Acetyltessellatine & $3064 *$ & 341 & $\begin{array}{l}341(41), 324(10), 323(10), 299(2), 280(8), 255(2), 254(4), 248(2), 238(2), 237(5), 236(17), \\
\text { 198(8), 181(4), 180(2), 156(7), 154(12), 138(22), 137(28), 136(27), 124(23), 120(63), 111(66), } \\
108(30), 106(61), 99(17), 94(35), 93(14), 80(100), 55(10), 53(10) .\end{array}$ & [42] \\
\hline 30 & 9-Acetytessellatine & $2962 *$ & 341 & $\begin{array}{l}341(7), 299(6), 282(11), 281(15), 248(3), 238(15), 237(16), 236(14), 198(18), 181(12), 180(10), \\
\text { 179(9), 153(13), 138(49), 136(45), 121(26), 120(100), 119(32), 108(11), 106(12), 94(52), } \\
93(89), 80(28), 53(11) .\end{array}$ & [42] \\
\hline
\end{tabular}


Table 3. Cont

\begin{tabular}{|c|c|c|c|c|c|}
\hline No. & Alkaloid & $\mathbf{R I}$ & {$[\mathbf{M}]^{+}$} & Characteristic ions $m / z$ (relative abundance) & References \\
\hline 31 & 3'-Acetyltrachelanthamine & - & 327 & $327(1), 284(2), 240(6), 184(6), 142(27), 125(38), 124(100), 84(14), 83(59), 82(54)$. & [128] \\
\hline 32 & 9-Acetyltrachelanthamidine & 1395 & 183 & 140(8), 125(8), 124(100), 110(5), $95(10), 83(50), 82(27), 55(23)$. & [40] \\
\hline 33 & 3'-Acetylviridiflorine & $2767 *$ & 327 & $\begin{array}{l}327(3), 284(6), 268(2), 267(2), 241(5), 240(9), 225(4), 184(4), 159(2), 142(21), 140(4), 125(17), \\
124(100), 83(14), 82(12), 55(19) .\end{array}$ & [42] \\
\hline 34 & $\begin{array}{l}\text { 9-(3'-Acetylviridifloryl) } \\
\text { retronecine }\end{array}$ & - & 341 & $341(4.7), 255(3), 182(5), 157(4), 138(100), 120(10), 93(92), 85(4), 83(20), 80(16), 43(38)$. & [119] \\
\hline 35 & $\begin{array}{l}\text { 9-(3'-Acetyl)viridifloryl } \\
\text { turneforcidine (or isomer) }\end{array}$ & $3050^{*}$ & 343 & $\begin{array}{l}343(5), 325(4), 300(12), 299(5), 257(14), 256(50), 212(13), 200(15), 197(14), 159(10), 158(36), \\
141(19), 140(30), 138(16), 122(21), 120(33), 117(10), 106(12), 96(44), 95(90), 83(11), 82(100), \\
69(17), 55(32) .\end{array}$ & [42] \\
\hline 36 & 7- Acetylvulgarine & - & 439 & $440\left(\mathrm{M}^{+}+1\right), 422(40), 380(15), 358(5), 340(65), 296(42), 282(85), 180(100)$ & [11] \\
\hline 37 & Amabiline & $\begin{array}{l}1985 \\
2652^{*}\end{array}$ & 283 & $\begin{array}{l}\text { 383(1), 140(8), } 123(30), 122(100), 121(46), 120(51), 108(17), 93(25), 80(13), 70(17), 53(7), \\
45(6), 43(19) .\end{array}$ & [67] \\
\hline 38 & Anadoline & - & 381 & $\begin{array}{l}381(6), 204(6), 167(6), 149(23), 139(22), 138(93), 137(45), 136(33), 120(17), 119(17), 118(13), \\
117(18), 111(12), 109(15), 100915), 97(17), 95(27), 94(55), 93(88), 85(25), 83(100), 82(17), \\
81(27), 80(27), 73(14), 71(37), 70(17), 67(27), 57(73), 56(17), 55(83), 54(13), 53(20) .\end{array}$ & [202] \\
\hline 39 & $\begin{array}{l}\text { 7a-Angeloyl-1-chloromethy- } \\
\text { 1,2-dihydropyrrolizidine }\end{array}$ & 1815 & 255 & $\begin{array}{l}220(40), 172(15), 155(45), 136(23), 130(24), 129(32), 128(63), 121(11), 120(94), 119(24), \\
106(30), 94(100), 93(20), 83(20), 80(17), 67(8), 55(35) .\end{array}$ & [70] \\
\hline 40 & $\begin{array}{l}\text { 7-Angeloyl-9-(2,3- } \\
\text { dihydroxybutyryl)heliotridine }\end{array}$ & 2333 & 339 & $\begin{array}{l}339(1), 324(1), 294(1), 239(6), 222(25), 221(25), 220(65), 219(8), 138(20), 137(10), 136(81), \\
121(24), 120(100), 119(85), 106(15), 94(50), 93(85), 83(24), 80(18), 75(2), 57(10), 55(25), \\
45(10) .\end{array}$ & [67] \\
\hline 41 & $\begin{array}{l}\text { 7-Angeloyl-9-(2,3- } \\
\text { dihydroxybutyryl)retronecine }\end{array}$ & 2315 & 339 & $\begin{array}{l}339(1), 239(5), 238(5), 237(5), 221(25), 220(99), 219(15), 141(20), 138(10), 137(11), 136 \\
(100), 121(15), 120(83), 119(34), 106(10), 94(55), 93(95), 83(41), 80(20), 75(2), 57(10), 55(40), \\
45(10) .\end{array}$ & [96] \\
\hline 42 & $\begin{array}{l}\text { 7-Angeloyl-9-(2,3- } \\
\text { dihydroxypropanoyl)retronecine }\end{array}$ & 2300 & 325 & $\begin{array}{l}294(0.5), 237(5), 255(9), 221(10), 220(67), 219(16), 141(22), 138(5), 137(11), 136(100), \\
121(11), 120(57), 119(40), 106(10), 94(62), 93(95), 83(37), 80(17), 55(32) .\end{array}$ & [40] \\
\hline 43 & $\begin{array}{l}\text { 7-Angeloyl-1-formyl-6,7- } \\
\text { dihydro-5H-pyrrolizidine }\end{array}$ & 1920 & 233 & $\begin{array}{l}215(3), 150(100), 134(92), 133(35), 122(4), 106(15), 105(38), 104(15), 83(10), 79(16), 55(20) \text {. } \\
46\end{array}$ & [67] \\
\hline 44 & $\begin{array}{l}\text { 7-Angeloyl-9- } \\
\text { (hydroxypropenoyl)retronecine }\end{array}$ & 2053 & 307 & $\begin{array}{l}221(13), 220(95), 207(24), 181(8), 141(30), 137(10), 136(100), 120(53), 119(24), 106(10), \\
94(63), 93(77), 83(43), 80(21), 67(9), 55(41), 43(26) .\end{array}$ & [40] \\
\hline
\end{tabular}


Table 3. Cont.

\begin{tabular}{|c|c|c|c|c|c|}
\hline No. & Alkaloid & RI & {$[\mathbf{M}]^{+}$} & Characteristic ions $m / z$ (relative abundance) & References \\
\hline 45 & 7-Angeloylechinatine & 2467 & 381 & $\begin{array}{l}336(2), 281(1), 238(11), 221(42), 220(86), 141(11), 138(11), 137(9), 136(55), 121(78), \\
\text { 120(100), 119(60), 117(4), 106(9), 99(3), } 94(30), 93(52), 83(17), 80(10), 55(17), 45(8), \\
43(21) .\end{array}$ & [67] \\
\hline 46 & 7-Angeloylheliotridine & 1820 & 237 & $\begin{array}{l}\text { 219(1), 154(2), 137(42), 136(20), 124(25), 111(35), 106(86), 94(25), 83(10), 80(100), 68(10), } \\
55(20) \text {. }\end{array}$ & [70] \\
\hline 47 & 7-Angeloylheliotine & - & 395 & 395(5), 295(7), 220(100), 136(57), 120(80), 119(70), 93(43), 83(34), 59(55), 43(18). & [127] \\
\hline 48 & 7-Angeloyllycopsamine & $2460 *$ & 381 & $\begin{array}{l}381(0.1), 336(0.9), 281(0.5), 238(0.5), 220(100), 136(65), 121(40), 120(90), 94(40), 93(85), \\
83(50), 80(15), 55(48) .\end{array}$ & [90] \\
\hline 49 & $\begin{array}{l}\text { 7-Angeloyl-9-(2- } \\
\text { methylbutyryl)heliotridine }\end{array}$ & 2180 & 321 & $\begin{array}{l}321(0.5), 221(43), 220(70), 195(5), 141(26), 138(4), 137(9), 136(85), 121(13), 120(100), \\
119(65), 106(13), 94(59), 93(71), 83(23), 80(15), 67(4), 57(27), 55(30) .\end{array}$ & [67] \\
\hline 50 & $\begin{array}{l}\text { 7-Angeloyl-9-(2- } \\
\text { methylbutyryl)retronecine }\end{array}$ & 2155 & 321 & $\begin{array}{l}221(35), 220(100), 195(5), 141(25), 138(3), 137(9), 136(90), 121(6), 120(53), 119(20), \\
106(8), 94(50), 93(70), 83(35), 80(15), 57(20), 55(35) .\end{array}$ & [96] \\
\hline 51 & 7-Angeloylretronecine & 1787 & 237 & $\begin{array}{l}\text { 237(2), } 219(3), 204(0.5), 191(1), 154(2), 138(5), 137(23), 136(18), 124(23), 111(38), 106(40), \\
94(20), 93(6), 83(11), 80(100), 55(22) .\end{array}$ & [96] \\
\hline 52 & 9-Angeloylretronecine & 1797 & 237 & $\begin{array}{l}\text { 237(1), 219(0.5), 193(3), 154(16), 138(32), 137(25), 136(10), 126(7), 120(2), 108(2), 94(25), } \\
93(100), 83(8), 80(10), 55(13) .\end{array}$ & [96] \\
\hline 53 & 7-Angeloylrinderine & 2465 & 381 & $\begin{array}{l}381(0.1), 336(2), 281(1), 238(10), 221(34), 220(80), 141(11), 138(10), 137(9), 136(53), \\
\text { 121(70), 120(100), 119(59), 117(4), 106(10), 94(28), 93(50), 83(15), 80(10), 55(17), 45(8), } \\
\text { 43(25). }\end{array}$ & [67] \\
\hline 54 & 9-Angeloyltrachelamthamidine & 1700 & 223 & 140(1), $125(30), 124(100), 123(20), 122(15), 110(4), 95(13), 83(45), 82(16), 70(6), 55(27)$. & [40] \\
\hline 55 & $\begin{array}{l}\text { 9-Angeloyl-7- } \\
\text { viridiflorylretronecine }\end{array}$ & - & 381 & $\begin{array}{l}220(8.3), 138(7.3), 137(9.8), 136(9.9), 120(28.6), 117(50), 106(29), 94(34), 93(23.1), 91(34.6), \\
79(40.8), 67(39.6), 64(81.8), 60(31), 58(57), 57(85), 55(100) .\end{array}$ & [60] \\
\hline 56 & Asperumine & - & 397 & $380,336,220,138,120$. & [203] \\
\hline 57 & 9-(Butyryl-2-ene) supinidine & $1674 * *$ & 207 & $\begin{array}{l}\text { 180(5), 159(7), 157(7), 122(100), 121(6), 120(23), 108(6), 93(15), 80(17), 71(25), 57(7), } \\
53(11), 45(11), 43(14) .\end{array}$ & [163] \\
\hline 58 & Canescine & - & 399 & $\begin{array}{l}\text { 399(0.4), 384(4), 355(1), 338(0.3), 256(10), 238(66), 220(21), 180(11.6), 136(42), 120(100), } \\
93(65), 80(20) .\end{array}$ & [154] \\
\hline 59 & Canescenine & - & 399 & $\begin{array}{l}399(0.1), 384(1.6), 355(0.4), 338(0.3), 256(9.6), 238(67.5), 220(20.6), 180(14.7), 136(45.1), \\
120(100), 93(61.6), 80(20.2) \text {. }\end{array}$ & [154] \\
\hline
\end{tabular}


Table 3. Cont.

\begin{tabular}{|c|c|c|c|c|c|}
\hline No. & Alkaloid & RI & {$[\mathbf{M}]^{+}$} & Characteristic ions $m / z$ (relative abundance) & References \\
\hline 60 & Coromandaline & - & 285 & 285(2), 267(7), 241(3), 240(9), 142(65), 125(23), 124(100), 83(28), 82(18). & [140] \\
\hline 61 & Coromandalinine & - & 283 & $\begin{array}{l}\text { 283(0.8), 239(0.4), 238(0.3), 140(7), 123(27), 122(100), 121(35), 120(40), 108(13), 94(8), } \\
\text { 93(20.5), }\end{array}$ & [140] \\
\hline 62 & Cryptanthine & & 353 & $354\left[\mathrm{M}^{+}+1\right](1), 272(2), 254(3), 238(2), 138(2), 120(100), 118(2)$. & [65] \\
\hline 63 & Curassanecine & - & 157 & 126(14), 98(10), 83(100) 82(23). & [117] \\
\hline 64 & Curassavine & - & 299 & 299(0.7), 281(1.4), 255(2.6), 254(5), 142(80), 125(27), 124(100). & [140] \\
\hline 65 & Curassavinine & - & 297 & $\begin{array}{l}\text { 297(0.3), 253(0.1), 252(0.1), 241(0.1), 239(0.1), 140(8), 123(31), 122(100), 121(44), 120(41), } \\
\text { 108(13), 94(6.4), 93(20). }\end{array}$ & [140] \\
\hline 66 & 9-Curassavoylheliotridine & 2190 & 313 & $\begin{array}{l}295(2), 269(0.5), 268(0.5), 251(0.5), 226(0.9), 156(8), 139(35), 138(100), 120(15), 111(9), \\
106(8), 95(25), 94(64), 93(80), 80(28), 67(13), 57(11), 45(8), 43(25) .\end{array}$ & [40] \\
\hline 67 & Cynaustine & - & 283 & $\mathrm{C}_{15} \mathrm{H}_{25} \mathrm{NO}_{4}, 122(100)$. & [68] \\
\hline 68 & Cynaustraline (or stereoisomer) & $2682 *$ & 285 & $\begin{array}{l}\text { 285(2), 267(4), 252(5), 242(1), 241(1), 240(3), 226(1), 142(34), 125(15), 124(100), 83(24), } \\
82(14), 55(25) .\end{array}$ & [42] \\
\hline 69 & Cynoglossamine & - & 445 & 147(100), 138(27), 119(17), 93(23). & [138] \\
\hline 70 & 5-Deoxylasiocarpine & - & 395 & $396[\mathrm{M}+\mathrm{H}]^{+}(2), 295(5.5), 363(1), 220(80), 120(83), 93(68), 83(32)$ & [123] \\
\hline $71 / 72$ & $\begin{array}{l}\text { 3',7-Diacetylintermedine/ } \\
\text { 3',7-Diacetyllycopsamine }\end{array}$ & 2340 & 383 & $\begin{array}{l}340(5), 297(10), 296(10), 181(22), 180(100), 136(18), 121(8), 120(47), 119(20), 101(10), \\
99(10), 94(18), 93(47), 80(5), 43(30) .\end{array}$ & [40] \\
\hline 73 & 3',9-Diacetyltessellatine & $3090^{*}$ & 383 & $\begin{array}{l}383(2), 342,341(37)(7), 324(21), 323(10), 297(8), 296(6), 280(10), 238(2), 237(6), 236(18), \\
\text { 198(10), 181(7), 180(4), 179(8), 154(14), 153(22), 138(20), 136(49), 121(18), 120(100), } \\
119(55), 108(12), 106(19), 99(13), 94(37), 93(82), 80(25), 53(11) .\end{array}$ & [42] \\
\hline 74 & $\begin{array}{l}\text { 5,6-Dihydro-7,9-dimethoxy-7H- } \\
\text { pyrrolizine }\end{array}$ & $1415^{* *}$ & 181 & 150(90), 134(5), 120(100), 119(35), 106(12), 91(3), 79(5). & [163] \\
\hline 75 & Dihydroechinatine & - & 301 & $\mathrm{MS}^{2}, 284,258,240,140,122,96$. & [167] \\
\hline 76 & $\begin{array}{l}\text { thero-2",3"- } \\
\text { Dihydroxyechiumine }\end{array}$ & - & 415 & 415(0.5), 371(2), 254(56), 210(26), 166(5), 138(30), 136(37), 120(100), 93(70). & [63] \\
\hline 77 & Dihydroxytriangularine & 2525 & 369 & $\begin{array}{l}338(1), 324(2), 269(30), 252(30), 237(40), 221(25), 220(100), 219(10), 141(20), 138(8), \\
\text { 137(10), 136(80), 121(20), 120(80), 119(30), } 106(8), 95(9), 94(45), 93(81), 83(39), 80(10), \\
55(28) .\end{array}$ & [40] \\
\hline
\end{tabular}


Table 3. Cont

\begin{tabular}{|c|c|c|c|c|c|}
\hline No. & Alkaloid & RI & {$[\mathbf{M}]^{+}$} & Characteristic ions $m / z$ (relative abundance) & References \\
\hline 78 & Dihydroxytriangularicine & 2525 & 369 & $\begin{array}{l}338(0.1), 324(0.1), 269(4), 252(4), 237(5), 221(24), 220(100), 219(3), 141(28), 138(6), 137(8), \\
136(67), 121(24), 120(75), 119(35), 106(8), 95(8), 94(47), 93(90), 83(41), 80(11), 55(24) .\end{array}$ & [40] \\
\hline 79 & Echihumiline & 2578 & 397 & $\begin{array}{l}397(0.1), 382(0.2), 352(0.1), 338(0.1), 321(0.1), 297(2), 238(2), 221(20), 220(100), 219 \\
(4), 138(6), 137(6), 136(40), 121(15), 120(39), 119(16), 106(4), 94 \text { (20), } 93 \text { (30), } 83(36), \\
80(6), 59(5), 55(6) .\end{array}$ & [91] \\
\hline 80 & Echimidine & 2560 & 397 & $\begin{array}{l}397(0.1), 382(0.1), 352(0.1), 297(2), 221(21), 220(100), 219(5), 138(5), 137(6), 136(48) \text {, } \\
121(26), 120(75), 119(30), 106(5), 94(30), 93(61), 83(39), 80(10), 59(10), 55(25), 43(18) .\end{array}$ & [96] \\
\hline 81 & Echimidine isomer (tigloyl) & 2580 & 397 & $\begin{array}{l}397(0.1), 382(0.1), 297(2), 238(3), 221(21), 220(100), 219(3), 138(5), 137(6), 136(48), \\
121(28), 120(62), 119(23), 106(5), 94(28), 93(46), 83(30), 80(10), 59(10), 55(20), 43(18) .\end{array}$ & [96] \\
\hline 82 & Echinatine & 2175 & 299 & $\begin{array}{l}\text { 284(0.1), 254(1), 156(10), 139(30), } 138(100), 137(7), 136(4), 95(9), 94(25), 93(46), 80(7) \text {, } \\
67(5), 53(3), 43(12) .\end{array}$ & [70] \\
\hline 83 & Echiumine & $3178^{*}$ & 381 & $\begin{array}{l}\text { 381(0.4), 338(1), 337(1), 336(1), 281(2), 255(1), 238(9), 237(2), 221(35), 220(100), 141(16), } \\
\text { 138(8), 136(47), 121(32), 120(69), 119(20), 106(7), 94(39), 93(50), 83(28), 80(17), 59(1), } \\
\text { 55(37), 53(9). }\end{array}$ & [42] \\
\hline 84 & Echiuplatine & & 381 & $\begin{array}{l}383\left[\mathrm{M}^{+}+1\right](3), 382(68), 365(1), 364(1), 322(2), 320(24), 300(5), 282(7), 280(1), 238(4), \\
220(51), 138(2), 120(100), 118(2) .\end{array}$ & [65] \\
\hline 85 & Echiupinine & - & 381 & $\begin{array}{l}381(0.3), 337(1), 336(1), 282(0.2), 281(2), 212(38), 220(100), 136(43), 121(33), 120(55), \\
119(19), 118(18), 117(14), 103(16), 94(24), 93(47),\end{array}$ & [95] \\
\hline 86 & Echivulgarine & - & 479 & $480\left(\mathrm{M}^{+}+1\right), 462(35), 418(10), 398(10), 380(90), 336(40), 322(100), 220(70)$. & [11] \\
\hline 87 & Ehretinine & - & 275 & $275,140(100), 123,97$. & [100] \\
\hline 88 & 2",3"-Epoxyechiumine & - & 397 & $\begin{array}{l}\text { 254(9), 237(33), 236(100), 164(17), 157(4), 138(4), 136(19), 121(24), 120(25), 94(27), } \\
\text { 93(44), 80(12), 71(4), 43(30). }\end{array}$ & [63] \\
\hline 89 & $\begin{array}{l}\text { Erythro-2",3"-chloro-2"- } \\
\text { hydroxyechiumine }\end{array}$ & - & 435 & $\begin{array}{l}\text { 435(0.5), 274(37), 273(32), 272(92), 254(10), 236(13), 208(9), 138(10), 136(25), 121(26), } \\
120(100), 94(32), 93(45), 80919), 71(19) .\end{array}$ & [63] \\
\hline 90 & $\begin{array}{l}1 \alpha-2 \alpha \text {-Epoxy- } 1 \beta \text { - } \\
\text { hydroxymethyl-8 } \alpha \text {-pyrrolizidine }\end{array}$ & - & 155 & 126(4), 124(13), 96(10), 80(4), 71(50, 70(100), 68(10), 67(5), 56(6), 55(65). & [106] \\
\hline 91 & Europine & 2217 & 329 & $\begin{array}{l}314(1), 256(5), 240(25), 239(20), 156(10), 139(20), 138(100), 120(5), 94(20), 93(50), 80(10), \\
67(4), 59(20), 43(5) .\end{array}$ & [52] \\
\hline
\end{tabular}


Table 3. Cont

\begin{tabular}{|c|c|c|c|c|c|}
\hline No. & Alkaloid & RI & {$[\mathbf{M}]^{+}$} & Characteristic ions $m / z$ (relative abundance) & References \\
\hline 92 & Floridanine & - & 441 & $426,397,382,168,152,151(100), 150,149,123,122,110,96,94$. & {$[62,204]$} \\
\hline 93 & Floridimine & - & 301 & 301(1), 239(8), 226(7), 142(36), 124(100), 95(12), 94(10), 83(55), 55(23). & [128] \\
\hline 94 & Floridine & - & 343 & $343(1), 328(2), 284(1), 240(2), 239(3), 225(10), 142(8), 124(100), 83(27)$. & [128] \\
\hline 95 & Floridinine & - & 301 & $\begin{array}{l}301(1), 242(5), 239(7), 226(5), 167(1), 142(46), 125(19), 124(100), 122(8), 110(6), 96(8), \\
95(7), 83(36), 82(24), 70(12), 59(12) .\end{array}$ & [128] \\
\hline 96 & Furcatine & $3138^{*}$ & 383 & $\begin{array}{l}383(0.2), 339(1), 281(1), 240(6), 223(35), 222(100), 143(18), 136(33), 121(27), 120(75), \\
119(21), 94(44), 93(65), 85(13), 80(19), 74(8), 73(5), 60(1), 57(46) .\end{array}$ & [42] \\
\hline 97 & Heleurine & 1970 & 297 & $\begin{array}{l}\text { 239(1), 198(3), 181(6), 140(15), 131(6), 123(30), 122(100), 120(60), 119(70), 108(16), } \\
93(17), 80(10), 70(11), 59(26) .\end{array}$ & [109] \\
\hline 98 & Helibracteatine & - & 255 & $\begin{array}{l}\text { 255(0.5), 238(6), 237(1), 224(9), 156(43.4), 155(45.5), 138(16.3), 122(2.3), 111(99.7), 99(31), } \\
98(100), 82(35) .\end{array}$ & [112] \\
\hline 99 & Helibractinecine & - & 173 & 173(22), 156(4), 155(11), 142(7), 129(10), 124(1), 112(5), 99(83), 98(83), 95(6), 83(7), 82(100). & [111] \\
\hline 100 & Helibracteatinecine & - & 173 & 173(20), 156(4.5), 155(14), 142(3), 129(12), 124(1), 112(5), 99(85), 98(100), 82(83.5). & [112] \\
\hline 101 & Helibracteatinine & - & 255 & $\begin{array}{l}\text { 255(2), 238(3), 237(3.5), 224(9), 198(7), 181(60), 156(16), 155(93), 154(10), 124(12), } \\
122(21), 100(23), 98(20), 83(23), 82(100) .\end{array}$ & [112] \\
\hline 102 & Helifoline & - & 255 & 237(3), 156(5), 138(11), 137(3), 112(27), 111(100), 99(9), 98(42), 94(41), 83(9), 82(20), 80(24). & [136] \\
\hline 103 & Helifolinecine & - & 173 & 155(9.5), 129(67), 124(3), 116(6), 112(8), 98(100), 82(59), 80(10). & [136] \\
\hline 104 & Helindicine & - & 281 & 281(35), 207(100), 191(15), 149(37), 135(33), 109(34), 97(73), 95(55), 83(57), 81(46). & [130] \\
\hline 105 & Heliocoromandaline & - & 285 & 285(1), 267(4), 252(4), 240(6), 142(41), 125(17), 124(100), 84(63), 83(20), 82(15). & [117] \\
\hline 106 & Heliocurassavine & - & 299 & 299(1), 284(1), 281(1), 255(3), 254(10), 252(8), 243(7), 226(8), 142(48), 124(100), 83(56), 82(40). & [117] \\
\hline 107 & Heliocurassavicine & - & 285 & 285(1), 267(3), 252(3), 240(3), 142(49), 125(22), 124(100), 84(63), 83(58), 82(41). & [117] \\
\hline 108 & Heliocurassavinine & - & 285 & 285(1), 267(4), 252(4), 240(3), 142(55), 125(24), 124(100), 84(22), 83(48), 82(48), 81(36). & [117] \\
\hline 109 & Heliospathine & - & 313 & $\begin{array}{l}313(1.3), 269(2), 268(1), 156(12), 139(43), 138(100), 137(15), 120(11), 95(20), 94(57), \\
93(75), 80(10) .\end{array}$ & [140] \\
\hline 110 & Heliospathuline & - & 299 & $\begin{array}{l}\text { 299(14.7), 282(6), 281(16.5), 238(18), 237(19), 236(20), 156(38), 139(30), 137(36), 136(20), } \\
\text { 124(19), 120(60), 111(100), 108(51), 106(63), 94(41), 93(13), 80(78), 45(12). }\end{array}$ & [140] \\
\hline 111 & Heliosupine & 2553 & 397 & $\begin{array}{l}382(0.1), 352(0.1), 297(2), 238(4), 221(29), 220(100), 141(11), 138(10), 137(8), 136(50), \\
121(40), 120(95), 119(70), 106(10), 94(26), 93(52), 83(12), 80(10), 59(10), 55(15), 43(15) .\end{array}$ & [70] \\
\hline
\end{tabular}


Table 3. Cont.

\begin{tabular}{|c|c|c|c|c|c|}
\hline No. & Alkaloid & RI & {$[\mathbf{M}]^{+}$} & Characteristic ions $m / z$ (relative abundance) & References \\
\hline 112 & Heliotridine & 1447 & 155 & 138(2), 111(55), 94(18), 80(100), 68(15). & [40] \\
\hline 113 & $\begin{array}{l}\text { Heliotridine } 2 \mathrm{~S}-\text { hydroxy-2S(1S- } \\
\text { hydroxyethyl-4-methyl- } \\
\text { pentanoyl ester }\end{array}$ & - & 313 & MS2: 269, 270, 224, 156, 138, 120, 94. & [50] \\
\hline 114 & Heliotrine & 2100 & 313 & $\begin{array}{l}\text { 255(0.3), 214(0.5), 197(1), 156(5), 139(22), 138(100), 136(13), 120(7), 94(41), 93(83), } \\
\text { 80(40), 59(85). }\end{array}$ & [52] \\
\hline 115 & Heliovicine & - & 285 & $\begin{array}{l}\text { 267(5), 252(5), 240(4), 226(3), 175(1), 142(35), 124(100), 96(11), 95(9), 83(25), 82(20), } \\
55(33) .\end{array}$ & [128] \\
\hline 116 & Heliscabine & - & 255 & 255(4), 156(42), 138(12), 111(100), 99(33), 98(92), 82(77), 80(10). & [139] \\
\hline 117 & Hydroxymyoscorpine & - & 397 & $\begin{array}{l}\text { 397(0.2), 382(0.5), 338(0.1), 297(3.6), 221(21.3), 220(100), 136(40), 121(16), 120(41), } \\
\text { 119(16.6), 94(18.4), 93(36). }\end{array}$ & [95] \\
\hline 118 & $\begin{array}{l}\text { 7-(Hydroxy-methylbutyryl)-9- } \\
\text { viridifloryl retronecine }\end{array}$ & $2560 * *$ & 399 & $\begin{array}{l}384(2), 354(0.4), 296(0.3), 282(2), 256(6), 239(21), 238(55), 138(15), 136(21), 121(35), \\
\text { 120(100), 108(2), 101(5), 95(10), 94(20), 93(42), 83(5), 80(6), 73(5), 67(3), 59(12), 55(0.5), } \\
\text { 43(25). }\end{array}$ & [59] \\
\hline 119 & Ilamine & - & 313 & 314(M $\left.\mathrm{M}^{+}+1,18\right), 224(6), 139(3), 122(52), 120(25), 93(20), 80(8), 59(100)$ & [115] \\
\hline 120 & Incanine & - & 337 & $\begin{array}{l}\text { 294(2), 250(4), 222(10), 206(8), 155(5), 136(100), 120(77), 119(85), 94(42), 93(56), 80(26), } \\
\text { 53(17), 43(69). }\end{array}$ & [46] \\
\hline 121 & Indicine & 2126 & 299 & 255(4), 156(11), 138(100), 93(95). & [158] \\
\hline 122 & $\begin{array}{l}\text { Isoechinatine }(9-(+)- \\
\text { viridiflorylheliotridine })\end{array}$ & - & 299 & 299(11), 156(10), 139(33), 138(100), 137(14), 94(32), 93(68), 80(18). & {$[75,138]$} \\
\hline 123 & Isolycopsamine & - & 299 & $\begin{array}{l}\text { 281(12.3), 236(10.3), 138(67), 137(42), 136(34), 124(21), 121(15), 120(70), 119(15), 118(15), } \\
\text { 117(14), 111(68), 110(21), 109(11), 108(49), 106(81), 95(17), 94(43), 93(28), 80(100). }\end{array}$ & [133] \\
\hline 124 & Isoretronocanol enantiomer & $1883^{*}$ & 141 & 141(20), 140(9), 124(13), 110(12), 108(11), 83(100), 82(56), 70(10), 68(11), 55(48). & [42] \\
\hline 125 & $\begin{array}{l}9-(3 '-\text { Isovaleryl)viridifloryl } \\
\text { retronecine }\end{array}$ & - & 383 & $\begin{array}{l}\text { 383(0.2), 255(3), 240(3), 223(5), 20194), 138(100), 120(10), 93(66), 85(28), 80(10), 57(19), } \\
\text { 43(10), }\end{array}$ & [119] \\
\hline 126 & Intermedine & 2133 & 299 & $\begin{array}{l}\text { 156(9), 139(35), 138(100), 137(13), 136(13), 120(10), 95(15), 94(50), 93(80), 80(14), 67(9), } \\
\text { 45(7), 43(18). }\end{array}$ & [40] \\
\hline 127 & Lactodine & - & 227 & $227(9), 138(32), 137(41), 124(15), 111(60), 106(21), 94(44), 80(100)$. & {$[76,187]$} \\
\hline
\end{tabular}


Table 3. Cont

\begin{tabular}{|c|c|c|c|c|c|}
\hline No. & Alkaloid & RI & {$[\mathbf{M}]^{+}$} & Characteristic ions $m / z$ (relative abundance) & References \\
\hline 128 & Lasiocarpine & - & 411 & $\begin{array}{l}\text { 396(2), 311(4), 279(5), 221(43), 220(11), 137(20), 136(49), 124(22), 120(74), 119(42), } \\
106(15), 95(49), 93(23), 93(33), 83(39) .\end{array}$ & [71] \\
\hline 129 & Latifoline & - & 393 & $\begin{array}{l}\text { 393(6), 293(9), 221(10), 220(74), 219(35), 137(11), 136(100), 120(49), 119(42), 118(8), } \\
94(33), 93(75), 83(29), 80(17), 67(9) \text {. }\end{array}$ & {$[103,104]$} \\
\hline 130 & 9-Latifolylretronecine & - & 311 & $312\left(\mathrm{M}^{+}+1,40\right), 195(40), 161(20), 119(100)$ & [101] \\
\hline 131 & Lepanthine & - & 315 & $\mathrm{MS}^{2}: 298,156,138,120,94$ & {$[166,167]$} \\
\hline 132 & Lindelofine (or steroisomer) & $2678^{*}$ & 285 & $\begin{array}{l}\text { 285(5), 267(5), 252(6), 242(1), 241(1), 240(1), 226(1), 142(30), 125(21), 124(100), 83(26), } \\
82(15), 55(30) .\end{array}$ & [42] \\
\hline 133 & Lithosenine & - & 415 & $\begin{array}{l}415(0.2), 400(10), 297(8), 256(3), 238(100), 237(15), 222(16), 220(24), 138(30), 137(8), \\
136(49), 121(36), 120(75), 119(36), 95(9), 94(34), 93(57), 80(12)\end{array}$ & [156] \\
\hline 134 & Longitubine & - & 353 & $\begin{array}{l}353(29), 293(12), 181(29), 180(78), 136(75), 120(69), 119(62), 118(15), 106(10), 101(22), \\
94(58), 93(100), 80(32), 67(17) \text {. }\end{array}$ & [104] \\
\hline 135 & Lycopsamine & 2145 & 299 & $\begin{array}{l}\text { 299(0.5), 254(1), 156(8), 139(31), } 138(100), 137(12), 136(12), 120(10), 108(4), 95(15), 94 \\
(55), 93(84), 80(14), 67(10), 45(8), 43(20) .\end{array}$ & [91] \\
\hline 136 & Macrophylline & - & 239 & $98,83(100), 55$ & [62] \\
\hline 137 & Megalanthonine & - & 301 & $\begin{array}{l}301(2), 283(2), 256(11), 240(8), 212(19), 158(86), 141(15), 140(43), 138(14), 124(10), \\
122(18), 114(13), 97(18), 96(44), 95(62), 83(15), 82(100), 55(32) .\end{array}$ & [134] \\
\hline 138 & 1-Methylene- $8 \alpha$-pyrrolizidine & 1274 & 139 & $139(15), 95(100)$. & [205] \\
\hline 139 & 7-(2-Methylbutyryl)retronecine & 1760 & 239 & $\begin{array}{l}\text { 239(20), 222(2), 154(20), 138(12), 137(12), 136(28), 124(37), 120(20), 111(28), 108(15), } \\
106(28), 94(30), 93(20), 80(100), 68(10), 57(19) .\end{array}$ & [96] \\
\hline 140 & 9-(2-Methylbutyryl)retronecine & 1795 & 239 & $\begin{array}{l}239(2), 195(5), 154(3), 138(98), 137(8), 136(5), 120(6), 108(5), 94(60), 93(100), 80(27), \\
67(15), 57(30) \text {. }\end{array}$ & [96] \\
\hline 141 & $\begin{array}{l}\text { 7-(2-Methylbutyryl)-9-(2,3- } \\
\text { dihydroxybutyryl)retronecine }\end{array}$ & 2285 & 341 & $\begin{array}{l}341(1), 239(10), 223(20), 222(100), 143(18), 138(11), 137(7), 136(50), 121(17), 120(80), \\
119(30), 106(8), 94(40), 93(83), 85(15), 80(15), 57(30), 45(9) .\end{array}$ & [91] \\
\hline 142 & $\begin{array}{l}\text { 7-(2-Methylbutyryl)-9- } \\
\text { echimidinyl retronecine }\end{array}$ & 2512 & 399 & $\begin{array}{l}399(0.1), 384(0.5), 354(0.5), 297(1), 223(20), 222(100), 221(5), 143(15), 138(8), 137(8), \\
136(40), 121(30), 120(95), 119(30), 106(9), 94(31), 93(70), 85(15), 80(15), 67(8), 59(15), \\
57(28), 45(8), 43(24) .\end{array}$ & [91] \\
\hline 143 & Monocrotaline & 2268 & 325 & $236(35), 136(46), 120(100), 119(64), 93(36), 80(12), 43(69)$. & [195] \\
\hline
\end{tabular}


Table 3. Cont.

\begin{tabular}{|c|c|c|c|c|c|}
\hline No. & Alkaloid & RI & {$[\mathbf{M}]^{+}$} & Characteristic ions $m / z$ (relative abundance) & References \\
\hline 144 & Myoscorpine & $3212 *$ & 381 & $\begin{array}{l}\text { 381(0.2), 338(1), 337(2), 336(2), 281(2), 255(1), 238(9), 237(3), 221(33), 220(100), 141(22), } \\
\text { 138(8), 136(46), 121(34), 120(71), 119(22), 106(8), 94(36), 93(55), 83(39), 80(19), 59(4), } \\
\text { 55(42), 53(11). }\end{array}$ & [42] \\
\hline 145 & Neolatifoline & - & 393 & $\begin{array}{l}\text { 393(7), 293(11), 221(16), 220(85), 219(31), 137(13), 136(100), 120(43), 119(48), 118(15), } \\
94(39), 93(79), 83(32), 80(16), 67(8) .\end{array}$ & [104] \\
\hline 146 & $(7 S, 8 R)$ Petranine & - & 285 & ESIMS (positive mode) $\mathrm{m} / \mathrm{z} 286.11966$ & [89] \\
\hline 147 & $(7 S, 8 S)$ Petranine & - & 285 & ESIMS (positive mode) $m / z 286.12162$ & [89] \\
\hline 148 & Platynecine & - & 157 & $113(18), 82(100), 68(18), 67(4), 55(27)$. & [106] \\
\hline 149 & $\begin{array}{l}\text { Platynecine N-Oxide } 2 \mathrm{~S}- \\
\text { hydroxy-2S(1S-hydroxyethyl)- } \\
\text { 4-methyl-pentanosyl ester }\end{array}$ & - & 331 & $314,288,174,156,112$. & {$[50]$} \\
\hline 150 & Pycnanthine & 2793 & 397 & $\begin{array}{l}397(0.8), 382(0.4), 354(2), 352(2), 281(3), 254(12), 237(32), 236(100), 235(7), 138(26), \\
\text { 137(14), 136(74), 121(46), 120(77), 119(20), 99(20), 94(50), 93(58), 80(20), 71(20), 58(15), } \\
\text { 45(20), 44(22), 43(20). }\end{array}$ & [91] \\
\hline 151 & Punctanecine & - & 383 & $\begin{array}{l}383(0.2), 368(0.5), 338(3), 296(2), 283(3), 266(4), 240(25), 222(19), 155(13), 140(45), \\
139(40), 137(73), 123(25), 122(48), 120(14), 96(23), 95(26), 83(27), 82(100) .\end{array}$ & {$[169,206]$} \\
\hline 152 & Retronecine & $\begin{array}{c}1430 \\
2190^{*}\end{array}$ & 155 & $155(26), 138(2), 111(55), 94(15), 80(100), 68(15)$. & [40] \\
\hline 153 & Retronecine dibenzoate & 2785 & 363 & 363(0), 258(10), 241(20), 136(43), $119(55), 105(100), 94(60), 93(78), 77(35)$. & [207] \\
\hline 154 & $\begin{array}{l}\text { Retronecine } 2 \mathrm{~S}-\text { hydroxy-2S(1S- } \\
\text { hydroxyethyl)-4-methyl- } \\
\text { pentanosyl ester }\end{array}$ & - & 313 & $\mathrm{MS}^{2}: 396,270,224,156,138$ & {$[50]$} \\
\hline 155 & $\begin{array}{l}\text { Retronecine } 2 \text { S-hydroxy-2S(1S- } \\
\text { hydroxyethyl)-[1'S- } \\
\text { hydroxyethyl)-4- } \\
\text { methylpentanoyl]-4-methyl- } \\
\text { pentanoyl ester, }\end{array}$ & - & 471 & ESIMS: $\mathrm{m} / \mathrm{z} 472[\mathrm{M}+\mathrm{H}]^{+}$, for $\mathrm{C}_{24} \mathrm{H}_{41} \mathrm{NO}_{8}$ & [51] \\
\hline 156 & Rinderine & 2155 & 299 & $\begin{array}{l}\text { 284(0.1), 254(0.6), 156(9), 139(35), 138(100), 137(10), 136(10), 120(5), } 95(12), 94(23) \text {, } \\
\text { 93(70), 80(12), 67(7), 53(5), 43(16). }\end{array}$ & [70] \\
\hline
\end{tabular}


Table 3. Cont

\begin{tabular}{|c|c|c|c|c|c|}
\hline No. & Alkaloid & RI & {$[\mathbf{M}]^{+}$} & Characteristic ions $m / z$ (relative abundance) & References \\
\hline 157 & Scorpioidine & - & 381 & $\begin{array}{l}381(7), 199(7), 139(11), 138(69), 137(28), 136(13), 120(9), 101(8), 95(4), 94(21), 93(41), \\
83(100), 80(9), 67(6), 57(6), 55(34), 53(6), 43(10) .\end{array}$ & [159] \\
\hline 158 & 7-Senecioylhelotridine & 1870 & 237 & 137(35), 136(18), 124(20), 111(35), 106(90), 94(21), 83(15), 80(100), 68(8), 55(18). & [70] \\
\hline 159 & 7-Senecioylretronecine & 1816 & 237 & $\begin{array}{l}\text { 237(4), 154(4), 137(30), 136(16), 124(25), 111(37), 106(40), 94(21), 83(23), 80(100), } 68(8) \\
55(15) .\end{array}$ & [91] \\
\hline 160 & 9-Senecioylretronecine & 1833 & 237 & $\begin{array}{l}\text { 237(1), 193(2), 155(11), 154(10), 138(23), 137(28), 136(15), 126(9), 109(5), 94(25), 93(100), } \\
83(20), 80(16), 67(6), 55(11) .\end{array}$ & [91] \\
\hline 161 & 7-Senecioylrinderine & 2515 & 381 & $\begin{array}{l}336(0.1), 221(28), 220(61), 138(10), 137(15), 136(50), 121(75), 120(100), 119(85), 106(23), \\
95(15), 94(48), 93(72), 83(27), 80(30), 55(40), 43(40)\end{array}$ & [40] \\
\hline 162 & 7-Senecioyllycopsamine & 2497 & 381 & $\begin{array}{l}336(0.5), 281(1), 238(5), 221(28), 220(100), 219(4), 141(15), 138(14), 137(12), 136(80), \\
121(38), 120(70), 119(20), 94(38), 93(61), 83(76), 80(11), 55(15), 45(8), 43(28) .\end{array}$ & [91] \\
\hline 163 & Sincamidine & - & 313 & $\mathrm{C}_{16} \mathrm{H}_{27} \mathrm{NO}_{5}$ (similar to heliotrine) & [44] \\
\hline 164 & Senkirkine & 2460 & 365 & 365(1), 266(10), 250(6), 222(9), 168(24), 153(41), 80(38), 43(100). & {$[54,195]$} \\
\hline 165 & Subulacine & - & 155 & 155(17), 126(4), 124(13), 96(10), 80(4), 71(5), 70(100), 68(10), 56(6), 55(65). & {$[106,208]$} \\
\hline 166 & Supinine & 1978 & 283 & $\begin{array}{l}\text { 283(0.2), 140(6), 123(25), 122(100), 121(40), 120(49), 108(11), 93(20), 80(8), 70(7), 53(5), } \\
45(4), 43(12) .\end{array}$ & [70] \\
\hline 167 & Supinidine & $1887^{*}$ & 139 & $\begin{array}{l}\text { 139(40), 138(12), 122(32), 120(14), 111(15), 110(18), 108(30), 94(13), 80(100), 70(4), } \\
68(17), 55(14), 53(17) .\end{array}$ & [42] \\
\hline 168 & $\begin{array}{l}\text { Supinidine N-oxide } 2 \text { S- } \\
\text { hydroxy-2S(1S-hydroxyethyl)- } \\
\text { 4-methyl-pentanoyl ester }\end{array}$ & - & 313 & $\mathrm{MS}^{2}: 296,270,224,156,138$ & {$[50]$} \\
\hline 169 & Symlandine & $3194 *$ & 381 & $\begin{array}{l}381(0.3), 338(1), 337(2), 336(2), 281(2), 255(1), 238(9), 237(3), 221(30), 220(100), 141(19), \\
\text { 138(10), 136(47), 121(35), 120(72), 119(21), 106(9), 94(36), 93(52), 83(31), 80(17), 59(3), } \\
\text { 55(40), 53(12). }\end{array}$ & [42] \\
\hline 170 & Symphytine & $3240 *$ & 381 & $\begin{array}{l}381(0.4), 338(1), 337(1), 336(3), 281(2), 255(1), 238(9), 237(2), 221(33), 220(100), 141(21), \\
\text { 138(7), 136(44), 121(33), 120(70), 119(20), 106(7), 94(36), 93(52), 83(33), 80(18), 59(3), } \\
55(38), 53(12) .\end{array}$ & {$[42]$} \\
\hline 171 & Symviridine & - & 381 & $\begin{array}{l}381(0.3), 337(0.1), 336(0.1), 281(0.2), 221(34.5), 220(100), 136(44.2), 121(22), 120(50), \\
119(11), 118(15), 117(7.6), 103(10), 95(23.5), 93(44.5) \text {. }\end{array}$ & [172] \\
\hline
\end{tabular}


Table 3. Cont

\begin{tabular}{|c|c|c|c|c|c|}
\hline No. & Alkaloid & RI & {$[\mathbf{M}]^{+}$} & Characteristic ions $m / z$ (relative abundance) & References \\
\hline 172 & Tessellatine & $2930^{*}$ & 299 & $\begin{array}{l}\text { 299(7), 281(7), 256(5), 255(3), 248(4), 238(11), 237(9), 236(11), 156(19), 139(25), 138(64), } \\
137(23), 124(16), 120(40), 111(45), 108(29), 106(39), 94(54), 93(40), 80(100), 53(17) .\end{array}$ & {$[42]$} \\
\hline 173 & $\begin{array}{l}\text { 7-Tigloyl-9-(2,3- } \\
\text { dihydroxybutyryl)retronecine }\end{array}$ & 2325 & 339 & $\begin{array}{l}239(5), 238(5), 237(8), 221(22), 220(90), 219(20), 141(20), 138(10), 137(11), 136(100), \\
\text { 121(15), 120(80), 119(35), 106(10), 94(58), 93(90), 83(46), 80(20), 75(2), 57(9), 55(40), } \\
45(10) \text {. }\end{array}$ & [96] \\
\hline 174 & $\begin{array}{l}\text { 7-Tigloyl-9-(2,3- } \\
\text { dihydroxypropanoyl)retronecine }\end{array}$ & 2320 & 325 & $\begin{array}{l}\text { 237(8), 225(12), 221(12), 220(70), 141(27), 138(5), 137(12), 136(93), 121(8), 120(55), } \\
119(48), 106(10), 94(59), 93(100), 83(55), 80(18), 55(24) .\end{array}$ & [40] \\
\hline 175 & 7-Tigloylheliotridine & 1873 & 237 & $\begin{array}{l}\text { 237(0.5), 137(55), 136(15), 124(19), 120(5), 111(41), 107(10), 106(100), 94(20), 86(6), } \\
83(12), 80(95), 68(5), 55(19) .\end{array}$ & [67] \\
\hline 176 & $\begin{array}{l}\text { 7-Tigloyl-9-( } \\
\text { hydroxypropenoyl) retronecine }\end{array}$ & 2073 & 307 & $\begin{array}{l}221(14), 220(100), 207(27), 181(10), 141(42), 137(10), 136(95), 120(53), 119(28), 106(10), \\
94(63), 93(84), 83(50), 80(20), 67(9), 55(37), 43(24) .\end{array}$ & [40] \\
\hline 177 & 7-Tigloyllycopsamine & $2473 *$ & 381 & $\begin{array}{l}336(1), 281(1.5), 238(1), 221(20), 220(100), 136(60), 121(30), 120(80), 94(40), 93(70), \\
83(50), 80(18), 55(40) .\end{array}$ & [90] \\
\hline 178 & $\begin{array}{l}\text { 7-Tigloyl-9-(2- } \\
\text { methybutyryl)retronecine }\end{array}$ & 2170 & 321 & $\begin{array}{l}\text { 221(36), 220(100), 195(5), 141(32), 138(2), 137(10), 136(82), 121(6), 120(53), 119(25), } \\
106(10), 94(53), 93(80), 83(40), 80(15), 57(20), 55(33) .\end{array}$ & [96] \\
\hline 179 & 7-Tigloylretronecine & 1816 & 237 & $\begin{array}{l}\text { 237(3), } 219(0.5), 154(2), 138(3), 137(29), 136(15), 124(25), 120(5), 111(44), 106(50), \\
94(20), 93(6), 83(15), 80(100), 55(22) .\end{array}$ & [96] \\
\hline 180 & 9-Tigloylretronecine & 1843 & 237 & $\begin{array}{l}\text { 193(5), 154(15), 138(20), 137(26), 136(13), 126(7), 119(5), 109(4), 94(23), 93(100), 83(10), } \\
80(12), 55(18) .\end{array}$ & [96] \\
\hline 181 & Trachelanthamidine & $1853^{*}$ & 141 & 141(24), 140(10), 124(15), 110(9), 108(7), 83(100), 82(62), 70(8), 68(8), 55(45). & [42] \\
\hline 182 & Trachelanthamine & 1970 & 285 & $\begin{array}{l}\text { 267(5), 252(4), 240(4), 142(50), } 125(18), 124(100), 110(4), 96(6), 83(20), 82(11), 70(6) \text {, } \\
55(14), 43(12) \text {. }\end{array}$ & [70] \\
\hline 183 & $\begin{array}{l}\text { Trachelanthamidine } 2 \mathrm{~S} \text { - } \\
\text { hydroxy-2S(1S-hydroxyethyl)- } \\
\text { 4-methyl-pentanoyl ester }\end{array}$ & - & 299 & ESIMS: $\mathrm{m} / \mathrm{z} 300[\mathrm{M}+\mathrm{H}]^{+}, 142,89,83,82,55$. & [51] \\
\hline 184 & 7-Trachelanthyl retronecine & $2899 *$ & 299 & $\begin{array}{l}\text { 299(12), 281(6), 236(11), 156(25), 138(50), 120(47), 111(45), 108(29), 106(40), 94(40), } \\
\text { 93(320, 80(100). }\end{array}$ & [42] \\
\hline 185 & Transalpinecine & - & 173 & $98,83,70$. & [145] \\
\hline
\end{tabular}


Table 3. Cont.

\begin{tabular}{|c|c|c|c|c|c|}
\hline No. & Alkaloid & RI & {$[\mathbf{M}]^{+}$} & Characteristic ions $m / z$ (relative abundance) & References \\
\hline 186 & Triangularine & 2375 & 335 & $\begin{array}{l}\text { 237(23), 236(6), 235(4), 221(5), 220(30), 219(18), 141(12), 137(12), 136(100), 121(28), } \\
120(64), 119(35), 95(12), 94(60), 93(98), 83(58), 80(15), 55(30) .\end{array}$ & [40] \\
\hline 187 & Triangularicine & 2394 & 335 & $\begin{array}{l}237(30), 236(6), 235(3), 221(5), 220(31), 219(10), 141(18), 137(10), 136(83), 121(27), \\
120(51), 119(40), 95(10), 94(55), 93(100), 83(60), 80(15), 55(25) .\end{array}$ & [40] \\
\hline 188 & Trichodesmine & 2388 & 353 & 353(1), 264(56), 222(5), 136(36), 120(100), 93(23), 81(16), 43(40). & [195] \\
\hline 189 & Turneforcidine & - & 157 & 113(20), 82(100), 68(21), 67(6), 55(32). & [106] \\
\hline 190 & Uluganine & & 399 & 399(2), 384(8), 356(1), 355(3), 354(2). & [183] \\
\hline 191 & Uplandicine & 2337 & 357 & $\begin{array}{l}357(4), 342(5), 297(23), 281(4), 256(4), 207(7), 206(52), 181(80), 180(100), 179(43), \\
\text { 136(75), 121(49), 120(85), } 119(50), 101(23), 94(55), 93(74), 80(28), 73(74), 59(23), 45(20), \\
44(69), 43(48) .\end{array}$ & [163] \\
\hline 192 & Viridinatine & - & 443 & 282(17), 138(41), 137(86), 124(32), 111(70), 106(42), 94(44), 80(100). & {$[76,187]$} \\
\hline 193 & Viridiflorine & 1983 & 285 & $\begin{array}{l}284(0.3), 267(5), 252(5), 240(5), 142(70), 125(23), 124(100), 117(3), \\
110(5), 83(35), 82(22), 70(15), 55(25), 43(21) .\end{array}$ & {$[67]$} \\
\hline 194 & $\begin{array}{l}\text { 9-Viridifloryl turneforcidine (or } \\
\text { isomer) }\end{array}$ & $2394 *$ & 301 & $\begin{array}{l}\text { 301(0.3), 283(8), 257(8), 256(14), 212(18), 159(21), 158(68), 141(17), 140(50), 138(32), } \\
\text { 122(24), 121(12), 120(31), 117(10), 106(13), 96(43), 95(81), 83(20), 82(100), 69(22), 55(40). }\end{array}$ & [42] \\
\hline 195 & Vulgarine & - & 397 & $398[\mathrm{M}+1]^{+}, 380(18), 336(5), 316(5), 298(40), 254(15), 240(52), 138(100), 120(14)$. & [11] \\
\hline
\end{tabular}

The RI reported for DB1 capillary column; * RI for DB-1701 capillary column; ** RI for OV1 capillary column; - not determined. 
Figure 16. Pyrrolizidine alkaloid structures in Boraginaceae. Compounds are numbered as in Table 3.

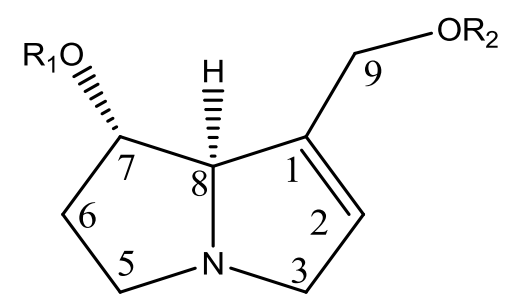

R1

$\mathrm{H}$

Ang

Tigl

Sen

Ac

$\mathrm{H}$

$\mathrm{H}$

Ang

Ac

$\mathrm{H}$

$\mathrm{H}$

$\mathrm{H}$

Ang

$\mathrm{H}$

Ac

$\mathrm{H}$

Ang

Sen

Ang

Ang

Ang

$\mathrm{H}$

$\mathrm{H}$

Ac

Ang

Ang

Ang

Ang

(-)Vir

$\mathrm{H}$

$\mathrm{H}$

\section{R2}

$\mathrm{H}$

$\mathrm{H}$

$\mathrm{H}$

$\mathrm{H}$

Cura

Cura

(-)-Vir

$(-)$-Vir

$(-)$-Vir

(-)-Vir,3'-OAc

$(+)$-Vir

Heliot

Heliot

(+)-Trcah

(+)-Trach

(+)-Trach,3'-OAc

(+)-Trcah

(+)-Trach

Ang

Ech

Ech, 3'-OAc

Las

Las, 5'-OAc

Las

Las

Las, 5'-OAc

Las, 2'-OAc

5'-Deoxy Las

$(-)$ Vir

(-)Lact

Vir or Trach, 3'-O-p-

hydroxycinammoyl

(+)-Trach, 5'-OH
Heliotridine (112)

7-Angeloylheliotridine (46)

7-Tigloylheliotridine (175)

7-Senecioylheliotridine (158)

7-Acetyl-9-curassavoylheliotridine (3)

9-Curassavoylheliotridine (66)

Echinatine (82)

7-Angeloylechinatine (45)

7-Acetylechinatine (7)

3'-Acetylechinatine (6)

Isoechinatine (122)

Heliotrine (114)

7-Angeloyheliotrine (47)

Rinderine (156)

7-Acetylrinderine

3'-Acetylreinderine (25)

7-Angeloylrinderine (54)

7-Senecioylrinderine (161)

Asperumine (56)

Heliosupine (111)

3'-Acetylheliosupine (13)

Europine (91)

5'-Acetyleuropine (10)

7-Acetyleuropine (11)

Lasiocarpine (128)

5'-Acetyllasiocarpine (18)

2'-Acetyllasiocarpine

5'-Deoxylasiocarpine (70)

Viridinatine (192)

Lactodine (127)

Cynoglossamine (69)

Canescine (58) 
Figure 16. Cont.

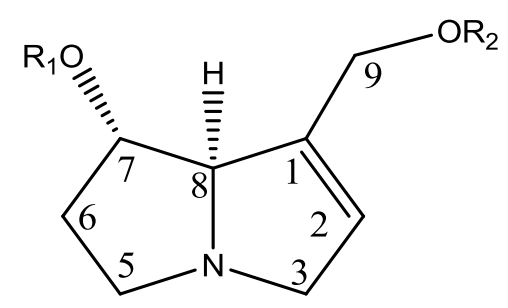

R1

3-Hydroxy-3-MeBut

3-Hydroxy-3-MeBut

3-Hydroxy-3-MeBut

Ang

Ang

3-Hydroxy-3-methyl butanoic

acid

Tigl

$\mathrm{H}$

$\mathbf{R}_{1}$

$\mathrm{H}$

Ac

Ac

Ang

$\mathrm{H}$

Sen

$\mathrm{H}$

Tigl

$\mathrm{H}$

Trach

$\mathrm{H}$

$\mathrm{H}$

Ac
R2

(+)-Trach, 5'-OH-3'-OAc

3'-Acetylcanescine (1)

(-)-Vir,5'-OH

Canescenine (59)

(+)-Vir, 5'-OH-3'-OAc

2,3-DiHydBut

3'-Acetylcasescenine (2)

7-Angeloy-9-(2,3-

dihydroxybutyryl)heliotridine (40)

2-MeBut

7-Angeloyl-9-(2-

methylbutyryl)heliotridine (49)

Vir. or its isomer Trach.

Uluganine (190)

(2-deoxy-2-methyl) Ech.

7-Tigloyl-9-(2-deoxy-2-

methyl)echimidinyl heliotridine.

2,3-dimethyl-2,3,4-

Onosmerectine

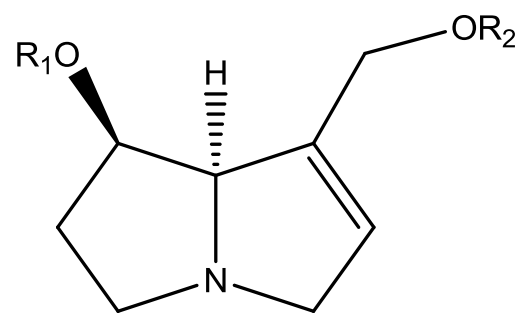

$\mathbf{R}_{\mathbf{2}}$

$\mathrm{H}$

$\mathrm{H}$

$t$ Sarr

$\mathrm{H}$

Ang

$\mathrm{H}$

Sen

$\mathrm{H}$

Tigl

$\mathrm{H}$

(+)-Trach

(+)-Trach,3'-OAc

$(+)$-Trach
Retronecine (152)

7-Acetylretronecine (24)

7-Acetyl-9-sarracinoylretronecine (26)

7-Angeloylretronecine (51)

9-Angeloylretronecine (52)

7-Senecioylretronecine (159)

9-Senecioylretronecine (160)

7-Tigloylretronecine (179)

9-Tigloylretronecine (180)

7-Trachelanthylretronecine (184)

Intermedine (126)

3'-Acetylintermedine (16)

7-Acetylintermedine (17) 
Figure 16. Cont.

\begin{tabular}{|c|c|c|}
\hline $\mathbf{R}_{\mathbf{1}}$ & $\mathbf{R}_{\mathbf{2}}$ & \\
\hline Ac & $(+)-$ Trach, 3'-OAc & 3',7-Diacetylintermedine (71) \\
\hline Ac & $(-)$-Vir,3'-OAc & 3',7-Diacetyllycopsamine (72) \\
\hline $\mathrm{H}$ & $(-)$-Vir & Lycopsamine (135) \\
\hline $\mathrm{H}$ & (-)-Vir,3'-OAc & 3'-Acetyllycopsamine (20) \\
\hline Ac & $(-)$-Vir & 7-Acetyllycopsamine (21) \\
\hline Ang & $(-)$-Vir & 7-Angeloyllycopsamine (48) \\
\hline Sen & $(-)$-Vir & 7-Senecioyllycopsamine (162) \\
\hline Tigl & $(-)$-Vir & 7-Tigloyllycopsamine (177) \\
\hline $\mathrm{H}$ & $(-)$-Trach & Indicine (121) \\
\hline Ac & $(-)$-Trach & 7-Acetylindicine \\
\hline $\mathrm{H}$ & $(-)$-Trach, 3'-OAc & 3'-Acetylindicine (15) \\
\hline Tigl & $(+)$-Trach & Myoscorpine (144) \\
\hline Tigl & (+)-Trach,3'-OAc & 3'-Acetylmyoscorpine (23) \\
\hline Tigl & $(+)$-Trach,5'-OH & Hydroxymyoscorpine (117) \\
\hline Ang & $(+)$-Trach & Echiumine (83) \\
\hline Ang & $(+)$-Trach, 3'-OAc & 3'-Acetylechiumine (8) \\
\hline threo-2",3"-dihydroxy Ang & $(+)$-Trach & threo-2",3"-Dihydroxyechiumine (76) \\
\hline 2",3"-epoxy Ang. & $(+)$-Trach & 2",3"-Epoxyechiumine (88) \\
\hline erythro-3"-chloro-2"-hydroxy & $(+)$-Trach & erythro-3"-Chloro-2"-hydroxyechiumine \\
\hline Ang & & (89) \\
\hline Sen & $(+)$-Trach & Echiupinine (85) \\
\hline Ang & Ech & Echimidine (80) \\
\hline Tigl & Ech & Echimidine isomer (tigloyl) (81) \\
\hline Ang & Ech,3'-OAc & 3'-Acetylechimidine (9) \\
\hline Ang & Ech,, ''-OAc $^{\prime}$ & 5'-Acetylechimidine \\
\hline $\mathrm{H}$ & Ech. & Echimiplatine \\
\hline Sen & Ech & Echihumiline (79) \\
\hline Sen-4-OH & $(+)$-Trach & Pcynanthine (150) \\
\hline Ang & t Sar & Triangularine (186) \\
\hline Tigl & t Sar & Triangularicine (187) \\
\hline Ang & $(-)$-Vir & Symlandine (169) \\
\hline Tigl & $(-)$-Vir & Symphytine (170) \\
\hline Sen & $(-)$-Vir & Symviridine (171) \\
\hline$(+)$-Vir & $\mathrm{H}$ & iso-Lycopsamine (123) \\
\hline
\end{tabular}


Figure 16. Cont.

\begin{tabular}{|c|c|c|}
\hline $\mathbf{R}_{\mathbf{1}}$ & $\mathbf{R}_{\mathbf{2}}$ & \\
\hline$(-)$-Vir & $\mathrm{H}$ & Heliospathuline (110) \\
\hline$(-)$-Vir & $\mathrm{H}$ & Tessellatine (172) \\
\hline (-)-Vir,3'-OAc & $\mathrm{H}$ & 3'-Acetyltessellatine (29) \\
\hline$(-)$-Vir & Ac & 9-Acetyltessellatine (30) \\
\hline$(-)-$ Vir,3'-OAc & $\mathrm{Ac}$ & 3',9-Diacetyltessellatine (73) \\
\hline $\mathrm{H}$ & $(-)$ Curss & Heliospathine (109) \\
\hline $\mathrm{H}$ & Vir,3'-OAc & 9-(3'-Acetyl)viridifloryl retronecine (34) \\
\hline $\mathrm{H}$ & Helio & Sincamidine (163) \\
\hline Ang & Latif & Latifoline (129) \\
\hline Ang & Latif isomer & Neolatifoline (145) \\
\hline $\mathrm{H}$ & Latif & 9-Latifolylretronecine (130) \\
\hline $\mathrm{H}$ & Hacke & Hackelidine \\
\hline Ac & Latif. & Longitubine (134) \\
\hline $\mathrm{H}$ & $(+)$-Trach,3'-OTigl & Anadoline (38) \\
\hline $\mathrm{H}$ & (-)-Vir,3'-OTigl & Scorpioidine (157) \\
\hline Ac & (-)-Vir,3'-OTigl & 7-Acetylscorpioidine (27) \\
\hline $\mathrm{H}$ & $(+)$-Trach, 5'-OH & Leptanthine (131) \\
\hline $\mathrm{H}$ & $(+)$-Trach, 5'-OAng & Vulgarine (195) \\
\hline Ac & $(+)$-Trach, 5'-OAng & 7-Acetylvulgarine (36) \\
\hline Ang & $(+)$-Trach, 5'-OAng & Echivulgarine (86) \\
\hline Ac & $(+)$-Trach, 5'-OH & Uplandicine (191) \\
\hline $\mathrm{H}$ & Vir,3'-OiVal & 9-(3'-Isovaleryl)viridifloryl retronecine (125) \\
\hline Vir & $\mathrm{H}$ & 7-Viridiflorylretronecine \\
\hline 3-Hydroxy-3-MeBut & $(+)-\mathrm{Vir}, 5^{\prime}-\mathrm{OH}-3^{\prime}-\mathrm{OAc}$ & 3'-Acetyllithosenine (19) \\
\hline 2-MeBut & $(+)$-Trach & Furcatine (96) \\
\hline 2-MeBut & $(+)$-Trach,3'-OAc & 3'-Acetylfurcatine (12) \\
\hline 3-Hydroxy-3-MeBut & (-)-Vir,5'-OH & Lithosenine (133) \\
\hline Benz & Benz & Retronecine dibenzoate (153) \\
\hline 2-Me-2,3-dihydroxy-But & Ang & Cryptanthine (62) \\
\hline
\end{tabular}


Figure 16. Cont.<smiles>CC(=O)OC(C)C(C)C(C)C(C)C(C)=O</smiles><smiles>CC(=O)C(O)C(C)CC(=O)C(O)C(C)O[18OH]</smiles><smiles>CC(=O)C(O)CO</smiles>

2-MeBut $=2-$ Methylbutric acid

2,3-DiMeBut = 2,3-Dimethylbutric acid

2-Hyd-3-MeBut = 2-Hydroxy-3-methylbutric acid

2,3-DiHydBut = 2,3-Dihydroxybutric acid

2,3-DiHydProp = 2,3-Dihydroxypropanoic acid

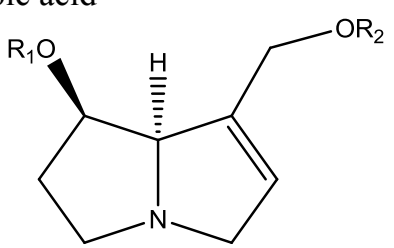

$\mathbf{R}_{1}$

2-MeBut

$\mathrm{H}$

2-MeBut

2-MeBut

Ang

Vir

Tigl

Ang

Tigl

Ang

Tigl

Ac

Ac

Ac

Ac

Ang

Tigl

Ang

Tigl

$$
\mathbf{R}_{2}
$$

$\mathrm{H}$

2-MeBut

2,3-DiHydBut

Ech

2-MeBut

Ang

2-MeBut

2-Hyd-3MeBut

2-Hyd-3MeBut

2,3-DiHydBut

2,3-DiHydBut

2-MeBut

2,3-DiMeBut

2-Hy-3MeBut

2,3-DiHydBut

2,3-DiHydProp

2,3-DiHydProp<smiles>CC(=O)C(O)(CO)C(C)O</smiles><smiles>CC(=O)C(O)(CO)C(C)O</smiles>

7-(2-Methylbutyryl)retronecine (139)

9-(2-Methylbutyryl)retronecine (140)

7-(2-Methylbutyryl)-9-(2,3-dihydroxybutyryl)retronecine (141)

7-(2-Methylbutyryl)-9-echimidinyl retronecine (142)

7-Angeloyl-9-(2-methylbutyryl)retronecine (50)

9-Angeloyl-7-viridifloryl retronecine (55)

7-Tigloyl-9-(2-methylbutyryl)retronecine (178)

7-Angeloy-9-(2-hydroxy-3-methylbutyryl)retronecine

7-Tigloyl-9-(2-hydroxy-3-methylbutyryl)retronecine

7-Angeloy-9-(2,3-dihydroxybutyryl)retronecine (41)

7-Tigloyl-9-(2,3-dihydroxybutyryl)retronecine (173)

7-Acetyl-9-(2-methylbutyryl)retronecine (22)

7-Acetyl-9-(2,3-dimethylbutyryl)retronecine (5)

7-Acetyl-9-(2-hydroxy-3-methylbutyryl)retronecine (14)

7-Acetyl-9-(2,3-dihydroxybutyryl)retronecine (4)

7-Angloyl-9-(2,3-dihydroxypropanoyl)retronecine (42)

7-Tigloyl-9-(2,3-dihydroxypropanoyl)retronecine (174)

Dihydroxytriangularine (77)

Dihydroxytriangularicine (78) 
Figure 16. Cont.

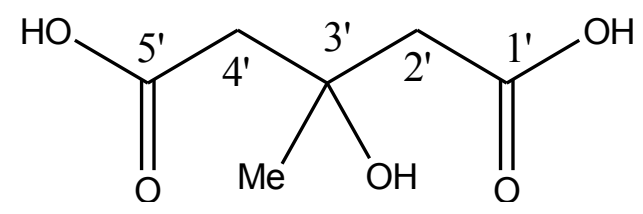

3-hydroxyl-3-methylglutaric acid

$\begin{array}{lll}\mathbf{R}_{\mathbf{1}} & \mathbf{R}_{\mathbf{2}} & \\ \text { Ang } & \text { 3-hydroxyl-3-methylglutaric acid } & \text { Echiuplatine (84) } \\ \text { Ang } & \text { 3-hydroxyl-3-methylglutaric acid methyl ester } & \text { Methylechiuplatine }\end{array}$

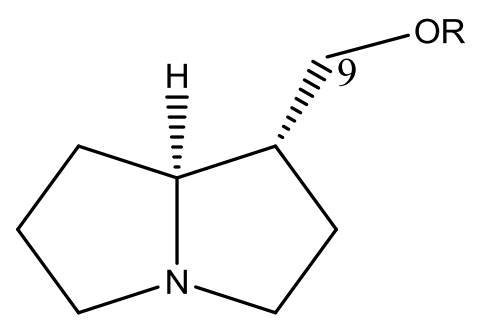

$\mathbf{R}$

$\mathrm{H}$

Ac

Ang

(+)-Trach

$(+)$-Trach, 3'-OAc

$(+)$-Vir

$(-)$-Curass

(-)-Vir,5'-OH

(-)-Vir,5'-OH-3'-OAc

(-)-Trach,5'-hydroxyl

(-)-Trach

$(-)$-Vir

(-)-Vir,3'-OAc

(2R,3R)-2,3-Dihydroxy-3-methylpentanoyl

Ech

$$
\text { (-)-Trachelanthamidine (181) }
$$

9-Acetyltrachelanthamidine (32)

9-Angeloyltrachelanthamidine (54)

Trachelanthamine (182)

3'-Acetyltrachelanthamine (31)

Coromandaline (60)

Curassavine (64)

Floridimine (93)

Floridine (94)

Floridinine (95)

Heliovicine (115)

Viridiflorine (193)

3'-Acetylviridiflorine (33)

Strigosine

Macrotomine 
Figure 16. Cont.

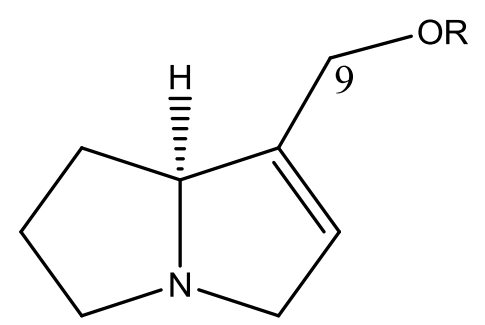

\section{$\mathbf{R}$}

$\mathrm{H}$

(+)-Trach,3'-OAc

$(-)$-Vir

$(+)$-Vir

$(-)$-Curass

Heliot

Las

(+)-Trach
(-)-Supinidine (167)

3'-Acetylsupinine (28)

Amabiline (37)

Coromandalinine (61)

Curassavinine (65)

Heleurine (97)

Ilamine (119)

Supinine (166)

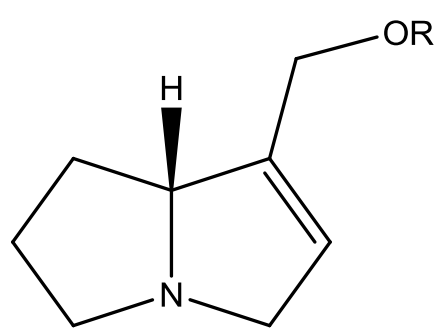

(+)-Supinidine (167)

Cynaustine (67)

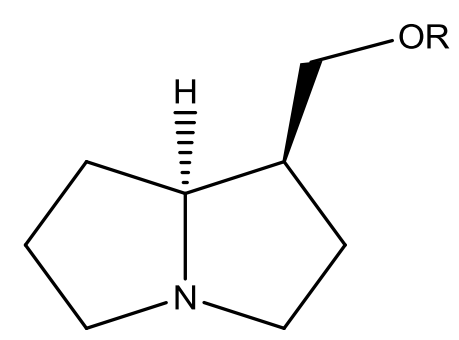

\section{$\mathbf{R}$}

$\mathrm{H}$

(+)-Vir

(-)-Curass

(-)-Trach
(-)-Isoretronecanol (124)

Heliocoromandaline (105)

Heliocurassavine (106)

Heliocurassavicine (107) 
Figure 16. Cont.

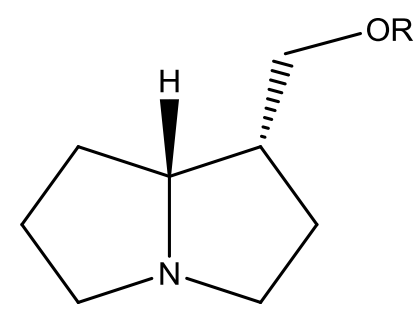

$\mathbf{R}$

$\mathrm{H}$

(-)Vir

(+)-Trach

(+)-Trach, 3'-OTigl

$p$-Hydroxycinnamoyl

4'- $p$-Hydroxy-cinnamoylglucoside
$(+)-$ Isoretronecanol [(+)-Lindelofidine] (124)

Cynaustraline (68)

Lindelofine (132)

Lindelofamine

Thesinine

Thesinine-4'-O- $\beta$-D-glucose

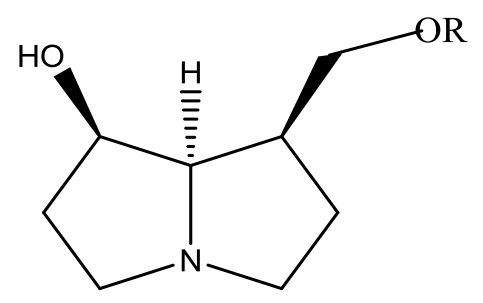

$\mathbf{R}$

$\mathrm{H}$

(-)-Vir

Platynecine (148)

Megalanthonine (137)

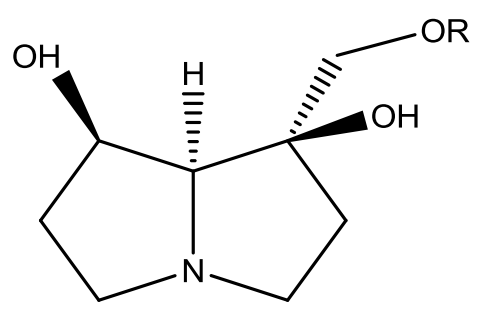

$\mathbf{R}$

$\mathrm{H}$

Helibractinecine (99)

Ang

Heliscabine (116)

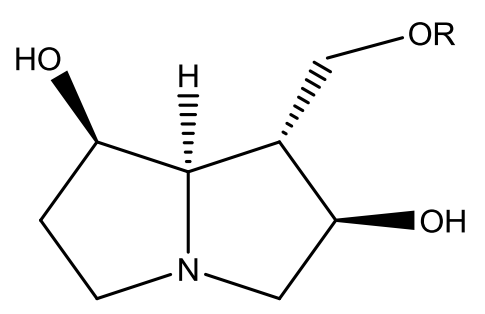

$\mathbf{R}$

$\mathrm{H}$

Helifolinecine or croalbinecine (103)

Ang

Helifoline (102) 
Figure 16. Cont.

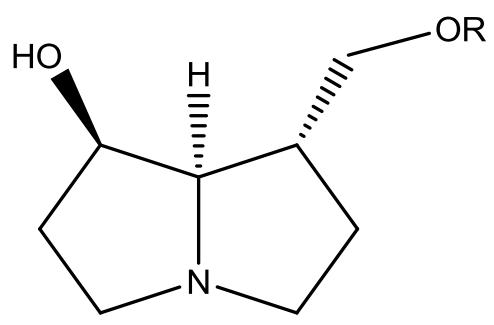

$\mathbf{R}$

$\mathrm{H}$

Vir, 3'-OAc

Vir
(-) Turneforcidine (189)

9-(3'-Acetyl)viridifloryl turneforcidine (35)

9-Viridifloryl turneforcidine (194)

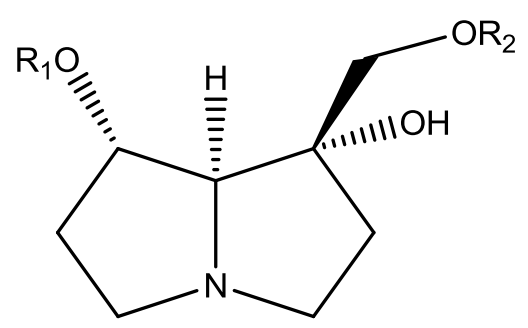

R1

$\mathrm{H}$

$\mathrm{H}$

Ang
R2

$\mathrm{H}$ Ang $\mathrm{H}$
Helibracteatinecine (100)

Helibracteatine (98)

Helibracteatinine (101)

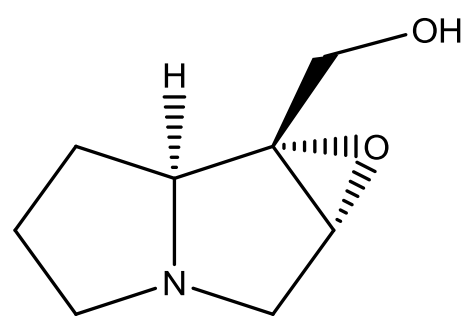

$1 \alpha, 2 \alpha$-Epoxy- $1 \beta$-hydroxymethyl-

$8 \alpha$-pyrrolizidine (90)

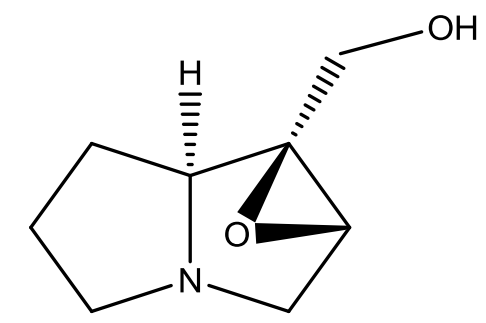

Subulacine (165)

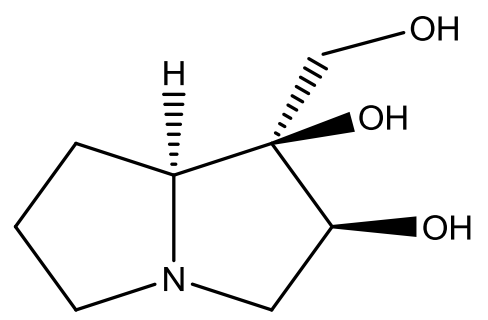

Transalpinecine (185)

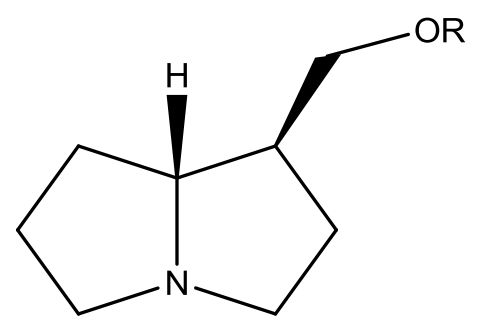

$\mathbf{R}$

$\mathrm{H}$

$(-)$-Trach

$(-)$-Vir

(+)-Trach
Laburnine [(+)-trachelanthamidine] (181)

Heliocurassavinine (108)

Neocormandaline

9-(+)-Trachelanthyl laburnine 
Figure 16. Cont.

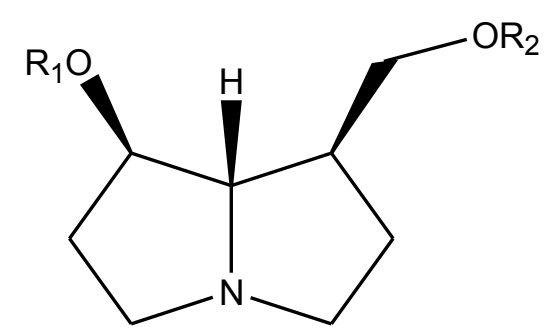

$\begin{array}{lll}\text { R1 } & \text { R2 } & \\ \text { H } & \text { H } & (+) \text {-Hastanecine } \\ \text { Ang } & (-) \text {-Vir } & \text { Punctanecine (151) }\end{array}$

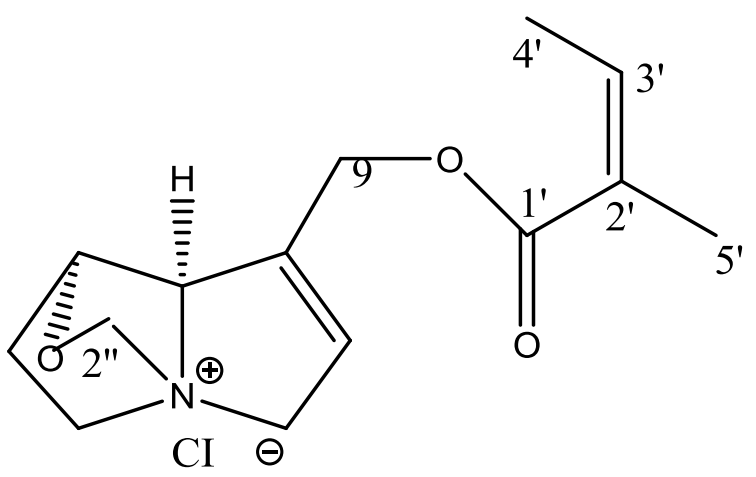

(7S, 8R)-Petranine (146)

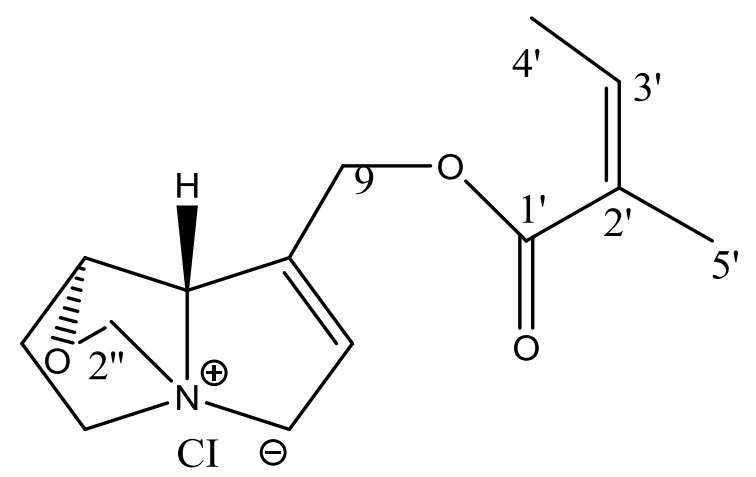

(7S, 8S)-Petranine (147)

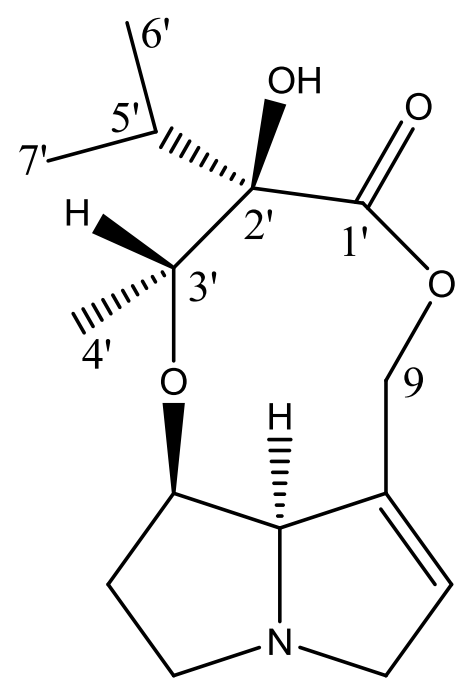

Helindicine (104)
R1

$\mathrm{H}$

$\mathrm{OH}$

$\mathrm{OH}$

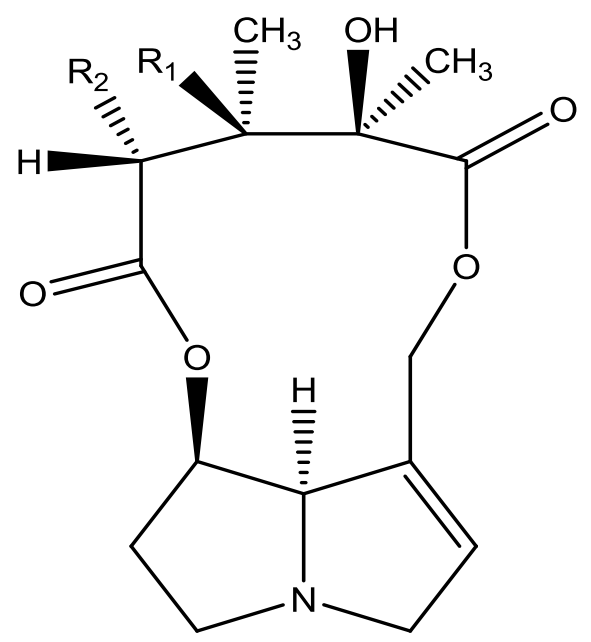

R2

$\mathrm{CH}\left(\mathrm{CH}_{3}\right)_{2}$

Incanine (120)

$\mathrm{CH}_{3}$

Monocrotaline (143)

$\mathrm{CH}\left(\mathrm{CH}_{3}\right)_{2}$

Trichodesmine (188) 
Figure 16. Cont.

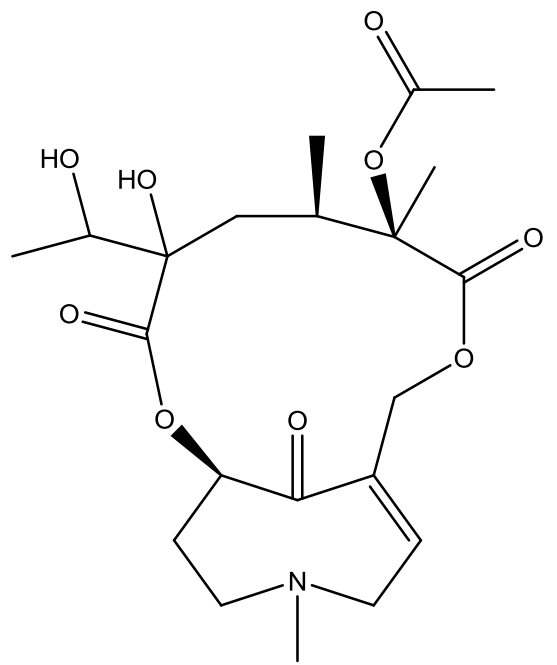

Floridanine (92)

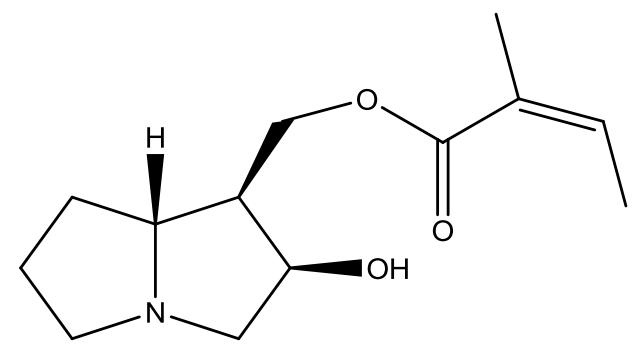

Macrophylline (136)

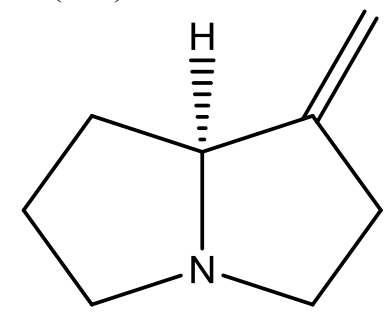

1-Methylene-8 $\alpha$-pyrrolizidine (138)

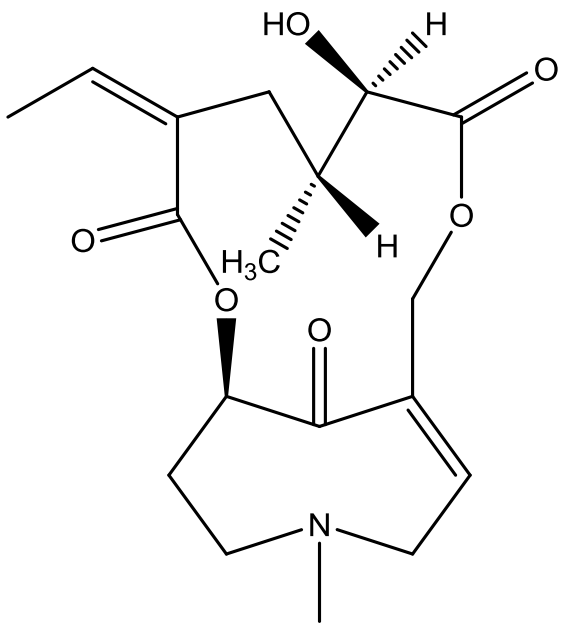

Senkirkine (164)<smiles>COc1ccc(C(=O)O[C@@H]2CCN3CC[C@H](C)[C@H]23)cc1</smiles>

Ehretinine (87)

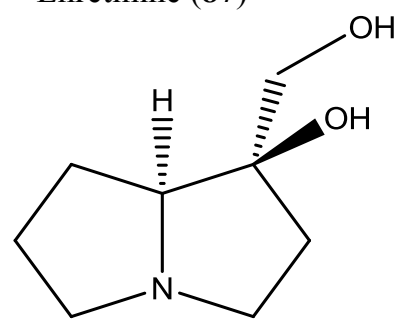

Curassanecine (63)

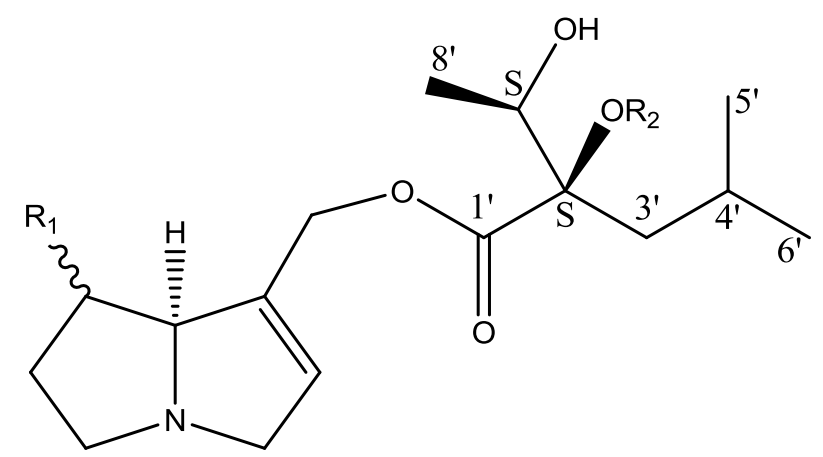

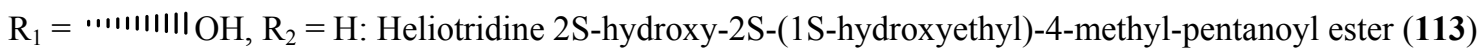

$\mathrm{R}_{1}=-\mathrm{OH} \mathrm{R}_{2}=\mathrm{H}$ : Retronecine 2S-hydroxy-2S-(1S-hydroxyethyl)-4-methyl-pentanoyl ester (154)

$\mathrm{R} 1=-\mathrm{OH}, \mathrm{R}_{2}=$ Erythro-2-hydroxy-2-(1-hydroxyethyl)-4-methyl-pentanoly: Retronecine 2S-hydroxy-2S-(1S-

hydroxyethyl)-2S-[1'S-hydroxyethyl)-4-methylpentanoyl]-4-methyl-pentanoyl ester (155)

$\mathrm{R}_{1}=\mathrm{H}, \mathrm{R}_{2}=\mathrm{H}$ : Supinidine N-Oxide 2S-hydroxy-2S-(1S-hydroxyethyl)-4-methyl-pentanoyl ester (168) 
Figure 16. Cont.

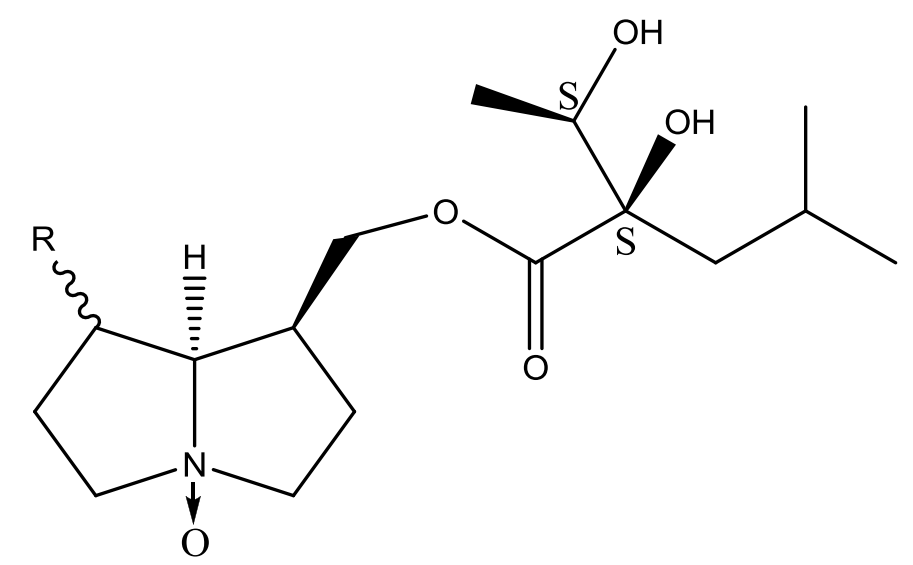

$\mathrm{R}=\mathrm{OH}$, Platynecine N-Oxide 2S-hydroxy-2S-(1S-hydroxyethyl)-4-methyl-pentanoyl ester (149)

$\mathrm{R}=\mathrm{H}$ : Trachelanthamidine 2S-hydroxy-2S-(1S-hydroxyethyl)-4-methyl-pentanoyl ester (183)

\subsubsection{Gas Liquid Chromatography-Mass Spectrometry (GLC-MS)}

High resolution Gas-liquid chromatography coupled with mass spectrometry (GLC-MS) has become a valuable and highly sensitive means for separation, convenient identification and quantification of complex PA mixtures, even in minute quantities or in diasteroisomeric forms [40,59,67,70,90,91,96,163,191-195]. The combination of molecular weight, group mass fragmentation pattern and Kovats retention indices, enables an unequivocal identification of most of PA tertiary bases even in trace amounts or of closely related isomers. High resolution gas chromatography-mass spectrometry (HRGC-MS) using the SIM mode was applied to detect trace amounts of 1,2-unsaturated PAs in 55 commercially available pollen products [16]. The detection limit of the overall procedure and the reliable quantitation limit were $0.003 \mu \mathrm{g} \mathrm{g}^{-1}$ to $0.01 \mu \mathrm{g} \mathrm{g}^{-1}$. GLC and GLC-MS cannot detect N-oxides, because they are not volatile. PA N-oxides need to be reduced to the free base (for example by reduction with zinc in $\mathrm{HCl}$ ) as an extra step in the sample preparation to make them suitable for GLC analysis.

\subsubsection{Liquid Chromatography-Mass Spectrometry (LC-MS)}

Coupling of liquid chromatography (LC) with mass spectrometry (MS) allows a simultaneous determination of PAs and their corresponding N-oxides in a single chromatographic run without the requirement of a reduction step [50]. Quantitative analysis of PA mixtures (e.g., from comfrey, Symphytum officinale) was carried out efficiently using electrospray liquid chromatography-mass spectrometry (LC-MS). The method is based on HPLC coupled to an ion-trap and orbitrap MS with electrospray ionization interface [177]. Plant samples from 9 Boraginaceous species have been screened with gradient HPLC equipped with diode array and electron impact mass spectrometry [60]. Several saturated and 1,2-unsaturated PAs (mainly as N-oxides) were detected using HPLC-ESI-MS in fresh pollen collected from flowers of the PAs producing plants Echium vulgare and E. plantagineum and /or from bee pollen from bees that had foraged on PA plants [12]. The same method has also been used to determine the PA profile in E. plantagineum [93], Cryptantha crassipes [64], and Anchusa strigosa [50]. PAs (N-oxides and free bases) occurring in small amounts could be efficiently analyzed using an RP-HPLC ion trap MS method with an atmospheric pressure chemical ionization (APCA) interface [167]. 
Most PAs in plants are present as N-oxides or their tertiary bases. The N-oxides of this class are polar, water soluble, non-volatile and thermally labile. Thus, the application of LC-MS to the analysis of PA $\mathrm{N}$-oxides has become a widely applied method $[64,177,199,209,210]$, for example, in honey samples.

\subsection{Nuclear Magnetic Resonance (NMR)}

For the determination of the type of necine, the sites of ester attachment and the stereochemical orientation of necic acids, NMR methods are essential. An excellent comprehensive review of ${ }^{1} \mathrm{H}$ NMR spectral data of PAs (more than 350 compounds) has been published by Logie et al. [211], in which the most useful shift values for the different types of PAs have been documented. Roeder [212] reported the ${ }^{13} \mathrm{C}$ NMR data of 136 PAs and updated his data in a book chapter of Rizk [28]. After that time, more NMR data have been published for new PAs which are documented in this review.

\subsection{Enzyme-Linked Immunoassay}

A highly sensitive and specific competitive enzyme-linked immunosorbent assay (ELISA) can be used for detection and quantification of PAs [213-216]. The limit of detection of this ELISA method was 1.9, 10, 18, 20 and $60 \mathrm{ng}$ for lasiocarpine, lasiocarpine N-oxide, heliotrine, and heliourine $\mathrm{N}$-oxide, respectively [217]. These data suggest that this technique can be an excellent tool to diagnose poisoned animals and identify PA contaminated food items.

\section{Tissue Culture}

Several Boraginaceae species were subjected to in vitro cultivation and their PA contents have been studied [153,218,219]. PA profiles of Hairy root cultures of Cynoglossum officinale and Symphytum officinale have been established [220]. In these cultures, all PAs are genuinely present as $N$-oxides. Recently, Abd El-Mawla [221] reported the influence of methyl jasmonate, quercetin and salicylic acid as elicitors of PA production in the hairy root cultures of Echium rauwolfii.

\section{Variation of PAs between Plant Organs and Developmental Stages}

Distributions of PAs vary within different plant organs. In Heliotropium indicum a tissue-specific PA analysis revealed the presence of PAs in all tissues: highest levels were detected in the inflorescences which contained more than $70 \%$ of total plant alkaloids [116,220]; the alkaloid contents of stems, leaves and roots were 3, 7 and 19\%, respectively. Young leaves, young inflorescences or seedlings showed high alkaloid levels, reaching 5\%-6\% dry matter in $H$. spanthulatum [116]. The total alkaloid contents of the root and rhizome of Pulmonaria obscura lay between 0.026 and $0.158 \mathrm{mg} / \mathrm{g}$ dry weight, whereas leaves and inflorescences accumulated only trace amounts of PAs (below $0.4 \mathrm{ng} / \mathrm{mg}$ dry weight) [168]. Extreme differences were found in PA levels between different leaves of Cynoglossum officinale [219]. The youngest leaves of the rosette plants had significantly higher PA contents than the oldest leaves (190 times). Alkaloid profiles and contents (total PAs and free bases) in the different plant organs of Paracaryum rugulosum, P. intermedium, Anchusa arvensis, Anchusa milleri, Alkanna orientalis, Alkanna tuberculata, Lappula spinocarpos and Trichodesma africana have been reported [40]. 
Table 4. ${ }^{13} \mathrm{C}$ NMR data of new pyrrolizidine alkaloids (PAs) in the time frame 1991-2013.

\begin{tabular}{|c|c|c|c|c|c|c|c|c|c|c|c|c|c|c|c|c|c|c|c|c|c|c|c|}
\hline Alkaloid & C-1 & $\mathrm{C}-2$ & C-3 & C-5 & C-6 & C-7 & $\mathrm{C}-8$ & C-9 & C-1' & C-2' & $\mathrm{C}-3^{\prime}$ & C-4' & $\mathrm{C}-5^{\prime}$ & C-6' & C-7' & C-1" & C-2" & C-3" & C-4" & C-5" & C-6" & solvent & References \\
\hline \multicolumn{24}{|l|}{ Necine Base } \\
\hline Helibractinecine & 82.8 & 38.3 & 53.4 & 52.5 & 36.2 & 73.7 & 71.2 & 68.6 & & & & & & & & & & & & & & $\begin{array}{l}\mathrm{CDCL}_{3-} \\
\mathrm{CD}_{3} \mathrm{OD}\end{array}$ & [111] \\
\hline Helibracteatinecine & 82.6 & 34.1 & 53.0 & 52.9 & 33.3 & 79.7 & 73.0 & 67.2 & & & & & & & & & & & & & & $\begin{array}{l}\mathrm{CDCL}_{3^{-}} \\
\mathrm{CD}_{3} \mathrm{OD}\end{array}$ & [112] \\
\hline Turneforcidine & 40.8 & 32.6 & 56.2 & 52.8 & 37.5 & 72.0 & 73.7 & 65.6 & & & & & & & & & & & & & & $\begin{array}{c}\mathrm{CD}_{3} \mathrm{OD}- \\
\mathrm{ND}_{3}\end{array}$ & [222] \\
\hline Transalpinecine & 82.3 & 84.4 & 59.2 & 55.9 & 28.0 & 24.6 & 72.3 & 63.9 & & & & & & & & & & & & & & $\mathrm{CD}_{3} \mathrm{OD}$ & [145] \\
\hline Subulacine & 70.9 & 63.4 & 54.0 & 58.4 & 27.1 & 26.2 & 67.9 & 60.2 & & & & & & & & & & & & & & $\mathrm{CD}_{3} \mathrm{OD}$ & [145] \\
\hline 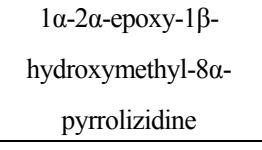 & 83.7 & 65.2 & 62.3 & 56.2 & 27.9 & 25.0 & 72.1 & 64.0 & & & & & & & & & & & & & & $\mathrm{CD}_{3} \mathrm{OD}$ & [145] \\
\hline \multicolumn{24}{|c|}{ Tertiary base and N-oxide alkaloids } \\
\hline 3'-Acetylcanescine & 133.0 & 127.5 & 62.6 & 53.6 & 34.4 & 74.4 & 75.4 & 62.3 & 175.1 & 84.3 & $\begin{array}{c}72.4- \\
\text { (CO:170.4), } \\
\text { (Me: } 21.2)\end{array}$ & 17.2 & 33.1 & 17.2 & 17.1 & 171.7 & 46.9 & 69.1 & 29.4 & 29.2 & & $\begin{array}{c}\mathrm{CDCL}_{3} / \mathrm{D}_{6} \\
-\mathrm{DMSO}\end{array}$ & {$[154]$} \\
\hline 3'-Acetylcanescenine & 133.0 & 127.5 & 62.6 & 53.6 & 34.4 & 74.4 & 75.4 & 62.3 & 175.1 & 84.3 & $\begin{array}{c}\text { 73.7- } \\
\text { (CO:170.4), } \\
\text { (Me: } 21.3 \text { ) }\end{array}$ & 16.0 & 33.1 & 17.8 & 14.2 & 171.7 & 46.9 & 69.1 & 29.4 & 29.2 & & $\begin{array}{c}\mathrm{CDCL}_{3} / \mathrm{D}_{6} \\
-\mathrm{DMSO}\end{array}$ & {$[154]$} \\
\hline $\begin{array}{l}\text { 3'-Acetylechihumiline } \\
\text { N-oxide }\end{array}$ & 133.0 & 124.9 & 79.4 & 70.5 & 33.9 & 73.3 & 95.6 & 63.0 & 174.7 & 85.6 & $\begin{array}{c}\text { 74.8- } \\
\text { (Me: 21.6) }\end{array}$ & 61.1 & 74.0 & 27.3 & 26.4 & 166.8 & 116.2 & 161.9 & 28.0 & 21.0 & & $\mathrm{CD}_{3} \mathrm{OD}$ & {$[166]$} \\
\hline $\begin{array}{l}\text { 7-Acetylechinatine } \mathrm{N} \text { - } \\
\text { oxide }\end{array}$ & 133.0 & 124.3 & 79.0 & 70.1 & 33.4 & 73.8 & 95.0 & 62.1 & 175.0 & 85.2 & 72.4 & 18.2 & 34.0 & 18.5 & 16.7 & 171.5 & 21.1 & & & & & $\mathrm{CD}_{3} \mathrm{OD}$ & [69] \\
\hline 7-Acetyleuropine & 135.0 & 128.3 & 62.3 & 54.4 & 30.6 & 76.9 & 78.8 & 62.6 & 174.2 & 84.0 & $\begin{array}{c}79.2- \\
\text { (OMe:56.8) }\end{array}$ & 13.3 & 73.4 & 26.7 & 24.9 & 171.3 & 21.5 & & & & & $\mathrm{CDCL}_{3}$ & [110] \\
\hline 3'-Acetylindicine & 132.3 & 130.7 & 62.8 & 53.0 & 36.1 & 71.1 & 78.5 & 63.3 & 174.5 & 81.6 & $\begin{array}{c}\text { 71.8- } \\
(\mathrm{CO}: 170.0) \\
(\mathrm{Me} 21.1)\end{array}$ & 14.3 & 32.3 & 17.2 & 16.3 & & & & & & & $\mathrm{CDCL}_{3}$ & [158] \\
\hline
\end{tabular}


Table 4. Cont.

\begin{tabular}{|c|c|c|c|c|c|c|c|c|c|c|c|c|c|c|c|c|c|c|c|c|c|c|c|}
\hline Alkaloid & C-1 & $\mathrm{C}-2$ & $\mathrm{C}-3$ & C-5 & C-6 & $\mathrm{C}-7$ & C-8 & C-9 & C-1' & C-2' & C-3' & C-4' & C-5 & C-6' & C-7' & C-1" & C-2" & C-3" & C-4" & C-5" & C-6" & solvent & References \\
\hline \multicolumn{24}{|c|}{ Tertiary base and N-oxide alkaloids } \\
\hline $\begin{array}{l}\text { 3'-Acetylindicine } \mathrm{N}- \\
\text { Oxide }\end{array}$ & 132.4 & 121.9 & 78.0 & 69.6 & 34.7 & 69.6 & 95.8 & 62.1 & 173.8 & 81.9 & $\begin{array}{c}\text { 72.4- } \\
\text { (CO:170.3), } \\
\text { (Me: } 21.2)\end{array}$ & 14.0 & 33.0 & 17.3 & 16.8 & & & & & & & $\mathrm{CDCL}_{3}$ & {$[158]$} \\
\hline 3'-Acetyllithonine & 132.5 & 128.2 & 62.2 & 53.0 & 33.8 & 73.5 & 74.8 & 61.5 & 172.3 & 82.9 & $\begin{array}{c}72.1- \\
\text { (CO:169.0), } \\
\text { (Me: 20.5) }\end{array}$ & 14.7 & 72.4 & 24.8 & 25.9 & 170.5 & 47.3 & 68.1 & 28.8 & 28.9 & & $\mathrm{CDCL}_{3}$ & {$[156]$} \\
\hline 3'-Acetylrinderine & 135.0 & 128.4 & 61.9 & 54.2 & 34.0 & 75.2 & 80.4 & 62.4 & 174.3 & 81.8 & $\begin{array}{c}\text { 72.5- } \\
\text { (CO:110.2), } \\
\text { (Me 13.9) }\end{array}$ & 21.1 & 33.1 & 16.8 & 17.2 & & & & & & & $\mathrm{CDCL}_{3}$ & [70] \\
\hline 3'-Acetyltrachelanthamine & 44.7 & 30.4 & 54.1 & 54.9 & 25.6 & 31.5 & 67.8 & 67.9 & 174.3 & 81.6 & $\begin{array}{c}72.2 \text {-(CO: } \\
\text { 169.7), } \\
\text { (Me: } 21.1)\end{array}$ & 13.9 & 32.8 & 16.7 & 17.2 & & & & & & & $\mathrm{CDCL}_{3}$ & [128] \\
\hline Canescine & 133.0 & 127.5 & 62.6 & 53.6 & 34.4 & 74.4 & 75.4 & 62.3 & 175.1 & 84.3 & 69.5 & 17.2 & 33.1 & 17.2 & 17.1 & 171.7 & 46.9 & 69.1 & 29.4 & 29.2 & & $\begin{array}{c}\mathrm{CDCL}_{3} / \mathrm{D}_{6} \\
\text {-DMSO }\end{array}$ & {$[154]$} \\
\hline Canescenine & 133.0 & 127.5 & 62.6 & 53.6 & 34.4 & 74.4 & 75.4 & 62.3 & 175.1 & 84.3 & 71.1 & 16.0 & 33.1 & 17.8 & 14.2 & 171.7 & 46.9 & 69.1 & 29.4 & 29.2 & & $\begin{array}{c}\mathrm{CDCL}_{3} / \mathrm{D}_{6} \\
\text {-DMSO }\end{array}$ & {$[154]$} \\
\hline Cryptanthine & 133.9 & 125.8 & 62.9 & 53.9 & 34.7 & 75.4 & 75.6 & 60.7 & 167.7 & 127.4 & 139.3 & 16.0 & 20.6 & 175.4 & 77.4 & 71.4 & 16.7 & 22.6 & & & & & {$[65]$. } \\
\hline Echihumiline & 132.8 & 127.6 & 62.0 & 53.7 & 34.2 & 73.7 & 76.1 & 62.0 & 174.3 & 83.1 & 69.8 & 18.5 & 72.4 & 26.0 & 24.9 & 165.5 & 115.3 & 159.0 & 27.7 & 20.4 & & $\mathrm{CDCL}_{3}$ & [91] \\
\hline Echimidine isomer & 133.3 & 129 & 63.2 & 54.1 & 34.8 & 74.2 & 76 & 62.9 & 174.7 & 83.7 & 70.0 & 18.9 & 73.8 & 26.5 & 25.2 & 167.5 & 128.9 & 138.2 & 14.8 & 12.3 & & $\mathrm{CDCL}_{3}$ & [87]. \\
\hline Echiupinine & 133.0 & 128.1 & 62.3 & 53.7 & 34.3 & 72.8 & 75.6 & 62.2 & 175.1 & 82.9 & 68.7 & 17.2 & 32.9 & 17.1 & 16.9 & 167.1 & 115.6 & 158.1 & 27.5 & 20.3 & & $\mathrm{CDCL}_{3}$ & [95] \\
\hline Echiupinine N-oxide & 131.8 & 128.1 & 78.2 & 69.1 & 34.3 & 71.4 & 93.7 & 60.2 & 174.9 & 84.0 & 69.4 & 16.9 & 33.2 & 17.5 & 17.2 & 166.2 & 114.5 & 160.3 & 27.6 & 20.6 & & $\mathrm{CDCL}_{3}$ & [95] \\
\hline $\begin{array}{l}\text { 7-Epi-echimiplatine } \mathrm{N}- \\
\text { oxide }\end{array}$ & 132.4 & 125.9 & 79.7 & 70.2 & 35.8 & 71.4 & 96.7 & 63.6 & 176.1 & 86.9 & 71.1 & 19.0 & 75.6 & 26.3 & 27.0 & & & & & & & $\mathrm{D}_{2} \mathrm{O}$ & {$[164]$} \\
\hline 2",3"-Epoxyechiumine & noise & 128.5 & 62.7 & 53.6 & 34.4 & 75.3 & 75.4 & 62.5 & 175.1 & 82.9 & 69.4 & 17.1 & 33.0 & 16.9 & 17.3 & noise & 77.2 & 60.0 & 13.6 & 19.3 & & $\mathrm{CDCL}_{3}$ & {$[63]$} \\
\hline $\begin{array}{l}\text { Erythro-3"-chloro-2"- } \\
\text { hydroxyechiumine }\end{array}$ & 132.6 & 127.8 & 62.4 & 53.7 & 34.5 & 75.4 & 75.8 & 62.3 & 173.6 & 83.0 & 69.4 & 16.9 & 33.0 & 17.1 & 17.3 & 175.1 & 77.2 & 62.8 & 17.9 & 23.0 & & $\mathrm{CDCL}_{3}$ & [63] \\
\hline
\end{tabular}


Table 4. Cont.

\begin{tabular}{|c|c|c|c|c|c|c|c|c|c|c|c|c|c|c|c|c|c|c|c|c|c|c|c|}
\hline Alkaloid & $\mathrm{C}-1$ & $\mathrm{C}-2$ & $\mathrm{C}-\mathbf{3}$ & C-5 & C-6 & C-7 & $\mathrm{C}-8$ & C-9 & C-1' & C-2' & $\mathrm{C}-3^{\prime}$ & C-4' & $\mathrm{C}-5$ & C-6' & C-7' & C-1" & C-2" & C-3" & $\mathrm{C}-4^{\prime \prime}$ & C-5" & C-6" & solvent & References \\
\hline \multicolumn{24}{|c|}{ Tertiary base and N-oxide alkaloids } \\
\hline & & & & & & & & & & & $72.7-(\mathrm{CO}:$ & & & & & & & & & & & & \\
\hline Floridine & 44.2 & 31.2 & 54.0 & 54.0 & 25.4 & 29.5 & 67.6 & 66.2 & 173.4 & 83.4 & $\begin{array}{c}\text { 169.6), } \\
\text { (Me: 21.2) }\end{array}$ & 15.1 & 73.5 & 26.2 & 24.9 & & & & & & & $\mathrm{CDCL}_{3}$ & {$[128]$} \\
\hline Floridinine & 43.3 & 28.6 & 53.8 & 54.0 & 24.6 & 30.6 & 68.4 & 66.2 & 174.6 & 82.5 & 69.8 & 18.6 & 73.9 & 25.0 & 26.0 & & & & & & & $\mathrm{CDCL}_{3}$ & {$[128]$} \\
\hline Floridimine & 44.3 & 28.7 & 54.2 & 54.3 & 25.3 & 30.6 & 67.6 & 63.2 & 174.7 & 84.1 & 69.9 & 18.6 & 73.8 & 26.0 & 24.9 & & & & & & & $\mathrm{CDCL}_{3}$ & [128] \\
\hline Helindicine & 134.2 & 125.3 & 62.5 & 55.5 & 36.7 & 70.5 & 80.5 & 61.7 & 175.6 & 84.5 & 70.5 & 17.3 & 34.2 & 17.2 & 17.6 & & & & & & & $\mathrm{CD}_{3} \mathrm{OD}$ & [130] \\
\hline $\begin{array}{l}\text { Heliotridine 2S- } \\
\text { hydroxy-2S-(1S- } \\
\text { hydroxyethyl)-4- } \\
\text { methyl-pentanoyl ester }\end{array}$ & 134.0 & 123.5 & 62.0 & 55.9 & 35.0 & 70.8 & 79.4 & 62.2 & $?$ & $?$ & 45.0 & 25.2 & 23.1 & 24.4 & $\begin{array}{c}73.9- \\
(8: 17.4)\end{array}$ & & & & & & & $\mathrm{CD}_{3} \mathrm{OD}$ & {$[50]$.} \\
\hline Heliscabine & 81.1 & 38.4 & 52.8 & 52.0 & 36.0 & 72.8 & 69.8 & 70.0 & 168.1 & 127.2 & 139.7 & 16.0 & 20.6 & & & & & & & & & $\mathrm{CDCL}_{3}$ & [139] \\
\hline Heliospathine & 133.1 & 128.9 & 62.4 & 53.9 & 36.1 & 70.5 & 78.2 & 62.2 & 174.5 & 84.4 & 71.2 & 17.4 & 38.9 & 24.7 & $\begin{array}{c}12.1- \\
\left(8^{\prime}: 11.9\right)\end{array}$ & & & & & & & $\mathrm{CDCL}_{3}$ & [140] \\
\hline Heliospathuline & 139.1 & 124.2 & 62.4 & 53.9 & 34.8 & 76.5 & 76.2 & 59.3 & & & & & & & & 174.3 & 83.8 & 72.4 & 16.5 & 31.9 & $\begin{array}{c}15.6 \\
\left(7^{7}: 17.2\right)\end{array}$ & $\mathrm{CDCL}_{3}$ & [140] \\
\hline Hydroxymyoscorpine & 132.8 & 128.5 & 62.8 & 53.7 & 34.3 & 73.8 & 75.7 & 62.5 & 174.2 & 83.0 & 69.6 & 18.4 & 73.5 & 25.9 & 24.8 & 167.1 & 128.6 & 137.8 & 14.4 & 11.9 & & $\mathrm{CDCL}_{3}$ & [95] \\
\hline $\begin{array}{l}\text { Isoechinatine }(9-(+)- \\
\text { viridiflorylheliotridine) }\end{array}$ & 136.1 & 126.3 & 61.7 & 54.3 & 33.8 & 74.7 & 79.8 & 61.9 & 174.3 & 83.9 & 71.6 & 17.5 & 32.2 & 17.8 & 15.9 & & & & & & & $\mathrm{CDCL}_{3}$ & {$[72,75]$} \\
\hline Iso-lycopsamine & 139.1 & 123.2 & 63.0 & 53.4 & 34.9 & 76.4 & 76.5 & 59.5 & & & & & & & & 174.1 & 82.8 & 69.4 & 16.5 & 33.38 & $\begin{array}{c}17.2 \\
(7: 1227)\end{array}$ & $\mathrm{CDCL}_{3}$ & [133] \\
\hline Lactodine & 138.1 & 123.2 & 63.8 & 54.1 & 35.1 & 69.4 & 76.5 & 58.9 & 173.3 & 68.4 & 16.3 & & & & & & & & & & & $\mathrm{CDCL}_{3}$ & [187] \\
\hline Leptanthine & 133.7 & 124.6 & 61.7 & 54.9 & 36.3 & 70.0 & 79.9 & 61.6 & 174.8 & 85.1 & 70.2 & 18.0 & 74.2 & 26.0 & 25.4 & & & & & & & $\mathrm{CD}_{3} \mathrm{OD}$ & [166]. \\
\hline Lithosenine $\mathrm{N}$-oxide & 133.7 & 124.0 & 78.0 & 69.7 & 36.3 & 71.2 & 96.8 & 62.5 & 175.5 & 86.1 & 71.2 & 19.1 & 75.2 & 27.0 & 26.3 & & & & & & & $\mathrm{CD}_{3} \mathrm{OD}$ & [166]. \\
\hline Lithosenine & 133.0 & 126.9 & 62.2 & 53.1 & 33.8 & 73.4 & 74.8 & 61.4 & 173.5 & 83.4 & 68.9 & 18.0 & 72.5 & 24.7 & 25.9 & 170.3 & 47.5 & 68.1 & 28.9 & 29.0 & & $\mathrm{CDCL}_{3}$ & {$[156]$} \\
\hline Megalanthonine & 36.0 & 31.2 & 51.1 & 54.6 & 36.8 & 69.5 & 72.8 & 66.2 & 174.5 & 84.1 & 72.0 & 17.0 & 32.0 & 15.6 & 17.8 & & & & & & & $\mathrm{CDCL}_{3}$ & [134] \\
\hline Methylechiuplatine & 133.5 & 128.0 & 62.9 & 54.0 & 34.7 & 73.6 & 76.1 & 61.3 & 171.3 & 44.9 & 69.7 & 44.8 & 172.2 & $\begin{array}{l}\text { 51.8: } \\
\text { OMe }\end{array}$ & 27.3 & 166.9 & 127.7 & 139.2 & 15.9 & 20.6 & & $\mathrm{CDCL}_{3}$ & [65] \\
\hline
\end{tabular}


Table 4. Cont.

\begin{tabular}{|c|c|c|c|c|c|c|c|c|c|c|c|c|c|c|c|c|c|c|c|c|c|c|c|}
\hline Alkaloid & C-1 & $\mathrm{C}-2$ & C-3 & C-5 & C-6 & $\mathrm{C}-7$ & C-8 & C-9 & C-1' & C-2' & C-3' & $C-4^{\prime}$ & C-5' & $\mathrm{C}-6^{\prime}$ & C-7' & C-1" & C-2" & C-3" & C-4" & C-5" & C-6" & solvent & References \\
\hline \multicolumn{24}{|c|}{ Tertiary base and N-oxide alkaloids } \\
\hline Myoscorpine & 133.1 & 128.1 & 62.7 & 53.8 & 34.4 & 73.7 & 75.8 & 62.2 & 175.1 & 82.9 & 69.3 & 17.2 & 32.9 & 17.1 & 16.9 & 167.0 & 128.5 & 137.8 & 14.4 & 11.9 & & $\mathrm{CDCL}_{3}$ & [95] \\
\hline Myoscorpine N-oxide & 131.8 & 124.0 & 78.4 & 69.3 & 34.3 & 72.4 & 93.8 & 60.1 & 14.9 & 84.0 & 69.4 & 16.9 & 33.1 & 17.5 & 17.2 & 166.2 & 127.9 & 139.0 & 14.6 & 12.0 & & $\mathrm{CDCL}_{3}$ & [95] \\
\hline $\begin{array}{l}\text { Onosmerectine N- } \\
\text { oxide }\end{array}$ & 133.5 & 123.8 & 78.6 & 69.7 & 35.7 & 70.7 & 96.7 & 62.5 & 175.3 & 85.7 & 74.6 & 70.8 & 18.7 & 26.6 & 25.9 & & & & & & & $\mathrm{CD}_{3} \mathrm{OD}$ & {$[164]$} \\
\hline (7S, 8R)-Petranine & 134.4 & 121.1 & 70.9 & 63.4 & 34.7 & 70.2 & 87.7 & 59.8 & 167.5 & 126.9 & 140.3 & 16.1 & 20.6 & & & & 68.8 & & & & & $\mathrm{CDCL}_{3}$ & [89] \\
\hline (7S, 8S)-Petranine & 134.6 & 121.3 & 71.2 & 63.6 & 34.8 & 70.6 & 88.1 & 59.9 & 167.0 & 127.0 & 149.9 & 16.3 & 20.8 & & & & 69.1 & & & & & $\mathrm{CDCL}_{3}$ & [89] \\
\hline Platynecine N-oxide & & & & & & & & & & & & & & & & & & & & & & & \\
\hline $\begin{array}{l}\text { 2S-hydroxy-2S-(1S- } \\
\text { hydroxyethyl)-4- } \\
\text { methyl-pentanoyl ester }\end{array}$ & 37.3 & 30.6 & 73.0 & 70.6 & 35.8 & 70.1 & 91.5 & 67.3 & $?$ & $?$ & 44.5 & 25.3 & 23.3 & 24.8 & $\begin{array}{c}73.9- \\
\left(8^{\prime}: 17.4\right)\end{array}$ & & & & & & & $\mathrm{CD}_{3} \mathrm{OD}$ & {$[50]$} \\
\hline Pycnanthine & 132.8 & 126.0 & 62.2 & 53.9 & 34.2 & 72.4 & 75.8 & 61.7 & 174.4 & 83.6 & 71.3 & 15.8 & 32.0 & 17.7 & 17.0 & 165.5 & 112.4 & 160.1 & 66.8 & 30.3 & & $\mathrm{CDCL}_{3}$ & [91] \\
\hline $\begin{array}{l}\text { Retronecine } 2 S- \\
\text { hydroxy-2S-(1S- } \\
\text { hydroxyethyl)-4- } \\
\text { methyl-pentanoyl ester }\end{array}$ & 133.2 & 123.8 & 62.0 & 54.0 & 36.7 & 70.0 & 80.0 & 61.0 & 175.4 & 82.3 & 45.2 & 25.3 & 23.3 & 24.6 & $\begin{array}{c}73.9- \\
(8: 17.6)\end{array}$ & & & & & & & $\mathrm{CD}_{3} \mathrm{OD}$ & {$[51]$} \\
\hline $\begin{array}{l}\text { Retronecine N-oxide } \\
\text { 2S-hydroxy-2S-(1S- } \\
\text { hydroxyethyl)-4- } \\
\text { methyl-pentanoyl ester }\end{array}$ & 133.9 & 123.4 & 78.8 & 70.0 & 35.7 & 70.6 & 97.2 & 62.4 & 175.9 & 82.0 & 45.0 & 25.2 & 23.3 & 24.6 & $\begin{array}{c}73.6- \\
(8 ': 17.7)\end{array}$ & & & & & & & $\mathrm{CD}_{3} \mathrm{OD}$ & [51] \\
\hline $\begin{array}{l}\text { Retronecine N-oxide } \\
\text { 2S-hydroxy-2S-(1R- } \\
\text { hydroxyethyl)-4- } \\
\text { methyl-pentanoyl ester }\end{array}$ & 134.0 & 123.4 & 78.8 & 70.0 & 35.8 & 70.6 & 97.0 & 62.0 & 175.5 & 81.0 & 44.0 & 25.8 & 23.9 & 24.3 & $\begin{array}{c}74.0- \\
(8: 16.5)\end{array}$ & & & & & & & $\mathrm{CD}_{3} \mathrm{OD}$ & {$[51]$} \\
\hline
\end{tabular}


Table 4. Cont.

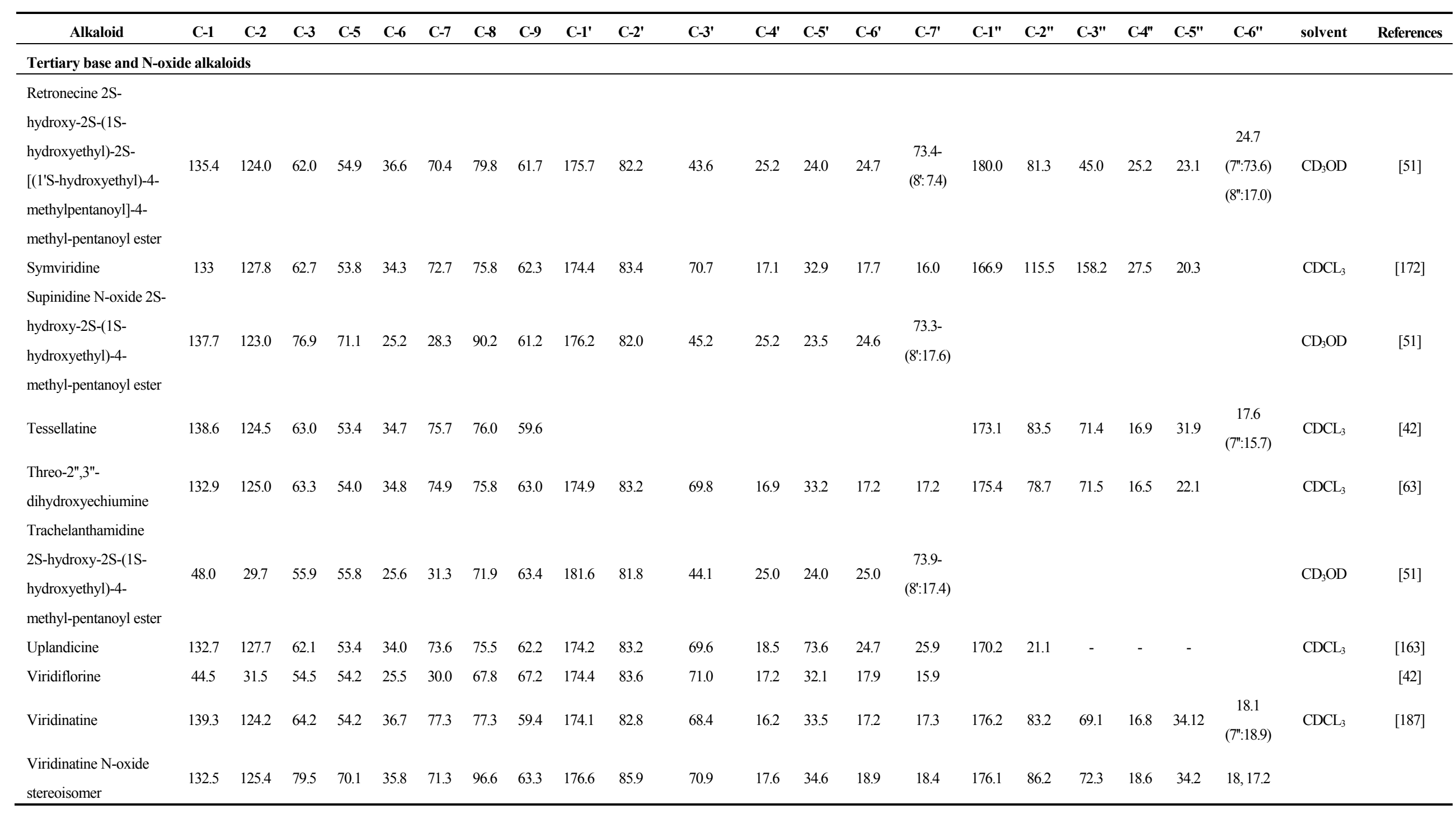


Table 5. ${ }^{1} \mathrm{H}$ NMR data of new PAs of in the time frame 1995-2013.

\begin{tabular}{|c|c|c|c|c|c|c|c|c|c|c|c|c|c|c|c|c|c|c|c|}
\hline Alkaloid & H-1 & H-2 & $\mathbf{H}-3$ & H-5 & H-6 & $\mathrm{H}-7$ & H-8 & $\mathrm{H}-9$ & H-3' & $\mathbf{H}-4^{\prime}$ & H-5' & H-6' & C-7' & H-2" & H-3" & H-4" & H-5" & solvent & References \\
\hline \multicolumn{20}{|l|}{ Necine Base } \\
\hline Helibractinecine & & \multirow{2}{*}{1.94} & $\alpha 3.13$ & $\alpha 3.07$ & \multirow{2}{*}{2.03} & \multirow{2}{*}{4.50} & \multirow{2}{*}{3.34} & $\alpha 3.60$ & & & & & & & & & & $\mathrm{CDCL}_{3^{-}}$ & \multirow{2}{*}{ [111] } \\
\hline$\left[\mathrm{C}_{8} \mathrm{H}_{15} \mathrm{NO}_{3}\right]$ & & & $\beta 2.93$ & $\beta 2.91$ & & & & $\beta 3.66$ & & & & & & & & & & $\mathrm{CD}_{3} \mathrm{OD}$ & \\
\hline Helibracteatinecine & & \multirow{2}{*}{1.63} & $\alpha 3.26$ & $\alpha 3.26$ & $\alpha 1.84$ & \multirow{2}{*}{3.94} & \multirow{2}{*}{3.12} & $\alpha 3.79$ & & & & & & & & & & $\mathrm{CDCL}_{3^{-}}$ & \multirow{2}{*}[112]{} \\
\hline$\left[\mathrm{C}_{8} \mathrm{H}_{15} \mathrm{NO}_{3}\right]$ & & & $\beta 2.55$ & $\beta 2.52$ & $\beta 2.11$ & & & $\beta 3.84$ & & & & & & & & & & $\mathrm{CD}_{3} \mathrm{OD}$ & \\
\hline Turneforcidine & \multirow{2}{*}{2.53} & $\alpha 1.68$ & $\alpha 3.08$ & $\alpha 3.03$ & $\alpha 2.00$ & \multirow{2}{*}{4.18} & \multirow{2}{*}{3.16} & $\alpha 3.57$ & & & & & & & & & & $\mathrm{CD}_{3} \mathrm{OD}-$ & \multirow{2}{*}[222]{} \\
\hline$\left[\mathrm{C}_{8} \mathrm{H}_{15} \mathrm{NO}_{2}\right]$ & & $\beta 2.10$ & $\beta 2.55$ & $\beta 2.70$ & $\beta 1.99$ & & & $\beta 3.53$ & & & & & & & & & & $\mathrm{ND}_{3}$ & \\
\hline Transalpinecine & & \multirow{2}{*}{4.18} & $\alpha 3.86$ & $\alpha 3.06$ & $\alpha 1.95$ & $\alpha 1.88$ & \multirow{2}{*}{3.99} & $\alpha 3.90$ & & & & & & & & & & \multirow{2}{*}{$\mathrm{CD}_{3} \mathrm{OD}$} & \multirow{2}{*}[145]{} \\
\hline$\left[\mathrm{C}_{8} \mathrm{H}_{15} \mathrm{NO}_{3}\right]$ & & & $\beta 3.38$ & $\beta 3.36$ & $\beta 2.16$ & $\beta 2.66$ & & $\beta 3.61$ & & & & & & & & & & & \\
\hline Subulacine & & \multirow{2}{*}{3.95} & $\alpha 3.79$ & $\alpha 2.97$ & $\alpha 1.96$ & $\alpha 2.19$ & \multirow{2}{*}{4.45} & $\alpha 3.97$ & & & & & & & & & & \multirow{2}{*}{$\mathrm{CD}_{3} \mathrm{OD}$} & \multirow{2}{*}[145]{} \\
\hline$\left[\mathrm{C}_{8} \mathrm{H}_{15} \mathrm{NO}_{2}\right]$ & & & $\beta 3.38$ & 3.62 & 2.24 & 2.26 & & $\beta 3.79$ & & & & & & & & & & & \\
\hline $1 \alpha-2 \alpha$-ероху- $1 \beta$ - & & & & & & & & & & & & & & & & & & & \\
\hline hydroxymethyl- $8 \alpha-$ & & \multirow{3}{*}{4.59} & $\alpha 3.81$ & $\alpha 3.23$ & $\alpha 2.08$ & $\alpha 2.00$ & \multirow{3}{*}{4.31} & $\alpha 3.93$ & & & & & & & & & & \multirow{3}{*}{$\mathrm{CD}_{3} \mathrm{OD}$} & {$[14=7$} \\
\hline pyrrolizidine & & & $\beta 3.89$ & $\beta 3.50$ & $\beta 2.23$ & $\beta 2.30$ & & $\beta 3.69$ & & & & & & & & & & & [145] \\
\hline$\left[\mathrm{C}_{8} \mathrm{H}_{15} \mathrm{NO}_{2}\right]$ & & & & & & & & & & & & & & & & & & & \\
\hline Tertiary base and N-oxi & alkaloid & & & & & & & & & & & & & & & & & & \\
\hline 3'-Acetylcanescine & & & $\alpha 3.95$ & $\alpha 3.33$ & & 5.20 & 425 & $\alpha 4.79$ & $5.21-$ & & 200 & 004 & 001 & 215 & & 126 & 126 & $\mathrm{CDCL}_{3} / \mathrm{D}$ & {$[151]$} \\
\hline$\left[\mathrm{C}_{22} \mathrm{H}_{35} \mathrm{NO}_{8}\right]$ & & 5.83 & $\beta 3.38$ & $\beta 2.65$ & 2.09 & 5.38 & 4.35 & $\beta 4.67$ & (COMe: 2.00) & 1.19 & 2.00 & 0.94 & 0.91 & 2.45 & & 1.26 & 1.26 & ${ }_{6}$-DMSO & [154] \\
\hline 3'-Acetylcanescenine & & 583 & $\alpha 3.95$ & $\alpha 3.33$ & 200 & 538 & 435 & $\alpha 4.74$ & $5.17-$ & 119 & 200 & 094 & 091 & 245 & & 126 & 126 & $\mathrm{CDCL}_{3} / \mathrm{D}$ & [151] \\
\hline$\left[\mathrm{C}_{22} \mathrm{H}_{35} \mathrm{NO}_{8}\right]$ & & (.0J & $\beta 3.38$ & $\beta 2.65$ & 2.09 & 5.50 & 4.JJ & $\beta 4.73$ & (COMe: 2.03) & 1.19 & 2.00 & 0.94 & 0.91 & $2.4 \mathrm{~J}$ & & 1.20 & 1.20 & 6-DMSO & {$[134]$} \\
\hline 3'-Acetylechihumiline & & & & $3.90-$ & & & & & & & & & & & & & & & \\
\hline N-oxide & & 6.03 & 8.51 & 3.75 & 02.78 & 5.76 & 4.83 & 4.72 & $5.40-$ & 1.34 & & 1.25 & 1.35 & 5.67 & - & 1.92 & 2.18 & $\mathrm{CD}_{3} \mathrm{OD}$ & {$[166]$} \\
\hline$\left[\mathrm{C}_{22} \mathrm{H}_{33} \mathrm{NO}_{9}\right]$ & & & p 4.09 & $(\mathrm{~m})$ & p 2.10 & & & & .98) & & & & & & & & & & \\
\hline 3'-Acetylechinatine & & 577 & $\alpha 3.37$ & $\alpha 2.68$ & $\alpha 1.91$ & 417 & 306 & $\alpha 4.78$ & $5.21-$ & 120 & 201 & 088 & 0.06 & & & & & CDCI & Г707 \\
\hline$\left[\mathrm{C}_{17} \mathrm{H}_{27} \mathrm{NO}_{6}\right]$ & & 0.10 & $\beta 3.92$ & $\beta 3.30$ & $\beta 1.98$ & 4.11 & 5.00 & $\beta 4.96$ & (COMe: 2.04) & 1.29 & 2.01 & 0.00 & 0.70 & & & & & $\mathrm{COC}_{3}$ & {$[10]$} \\
\hline 7-Acetylechinatine N- & & 600 & $\alpha 4.70$ & $\alpha 3.83$ & $\alpha 2.74$ & 569 & 488 & 478 & 396 & & 217 & 0.90 & 093 & 206 & & & & CDOD & [164] \\
\hline oxide & & 6.00 & $\beta 4.38$ & $\beta 3.79$ & $\beta 2.23$ & 5.69 & 4.88 & 4.78 & 3.96 & 1.42 & 2.17 & 0.90 & 0.93 & 2.06 & & & & $\mathrm{CD}_{3} \mathrm{OD}$ & [164] \\
\hline
\end{tabular}


Table 5. Cont.

\begin{tabular}{|c|c|c|c|c|c|c|c|c|c|c|c|c|c|c|c|c|c|c|c|}
\hline Alkaloid & H-1 & $\mathbf{H}-2$ & H-3 & H-5 & H-6 & H-7 & H-8 & H-9 & $\mathbf{H}-\mathbf{3}^{\prime}$ & $\mathbf{H}-4^{\prime}$ & $\mathbf{H}-\mathbf{5}^{\prime}$ & H-6 & C-7' & H-2" & H-3" & H-4" & H-5" & solvent & References \\
\hline \multicolumn{20}{|c|}{ Tertiary base and N-oxide alkaloids } \\
\hline $\begin{array}{l}\text { 7-Acetyleuropine } \\
{\left[\mathrm{C}_{18} \mathrm{H}_{29} \mathrm{NO}_{7}\right]}\end{array}$ & & 5.81 & $\begin{array}{l}\alpha 4.02 \\
\beta 3.38\end{array}$ & $\begin{array}{l}\alpha 3.28 \\
\beta 2.86\end{array}$ & 1.93 & 5.09 & 4.16 & $\begin{array}{l}\alpha 4.93 \\
\beta 4.87\end{array}$ & $\begin{array}{c}\text { 3.81- } \\
\text { (OMe: } 3.27)\end{array}$ & 1.27 & & 1.19 & 1.31 & 2.06 & & & & $\mathrm{CDCL}_{3}$ & {$[110]$} \\
\hline $\begin{array}{l}\text { 3'-Acetylindicine } \\
{\left[\mathrm{C}_{17} \mathrm{H}_{27} \mathrm{NO}_{6}\right]}\end{array}$ & & 5.92 & $\begin{array}{l}\alpha 3.96 \\
\beta 3.45\end{array}$ & $\begin{array}{l}\alpha 3.29 \\
\beta 2.76\end{array}$ & $\begin{array}{l}\alpha 1.98 \\
\beta 2.02\end{array}$ & 4.30 & 4.21 & 4.80 & $\begin{array}{c}5.20- \\
\text { (COMe: 2.04) }\end{array}$ & 1.23 & 2.09 & 0.95 & 0.92 & & & & & $\mathrm{CDCL}_{3}$ & {$[158]$} \\
\hline $\begin{array}{l}\text { 3'-Acetylindicine N-Oxide } \\
{\left[\mathrm{C}_{17} \mathrm{H}_{27} \mathrm{NO}_{7}\right]}\end{array}$ & & 5.79 & 4.45 & $\begin{array}{l}3.73- \\
3.80\end{array}$ & $\begin{array}{l}\alpha 2.01 \\
\beta 2.62\end{array}$ & 4.70 & 4.66 & 4.83 & $\begin{array}{c}5.23 \text { - } \\
\text { (COMe: 2.03) }\end{array}$ & 1.24 & 2.05 & 0.97 & 0.91 & & & & & $\mathrm{CDCL}_{3}$ & {$[158]$} \\
\hline $\begin{array}{l}\text { 3'-Acetyllithosenine } \\
{\left[\mathrm{C}_{22} \mathrm{H}_{35} \mathrm{NO}_{9}\right]}\end{array}$ & & 5.75 & $\begin{array}{l}\alpha 3.77 \\
\beta 3.29\end{array}$ & $\begin{array}{l}\alpha 3.17 \\
\beta 2.54\end{array}$ & 1.96 & 5.23 & 4.16 & $\begin{array}{l}\alpha 4.68 \\
\beta 4.59\end{array}$ & $\begin{array}{c}5.28- \\
\text { (COMe: 1.87) }\end{array}$ & 1.13 & & 1.25 & 1.11 & 2.33 & & 1.17 & 1.17 & $\mathrm{CDCL}_{3}$ & {$[156]$} \\
\hline $\begin{array}{l}\text { 3'-Acetylrinderine } \\
{\left[\mathrm{C}_{17} \mathrm{H}_{27} \mathrm{NO}_{6}\right]}\end{array}$ & & 5.76 & $\begin{array}{l}\alpha 3.37 \\
\beta 3.92\end{array}$ & $\begin{array}{l}\alpha 2.68 \\
\beta 3.30\end{array}$ & $\begin{array}{l}\alpha 1.91 \\
\beta 1.98\end{array}$ & 4.21 & 3.96 & $\begin{array}{l}\alpha 4.66 \\
\beta 4.93\end{array}$ & $\begin{array}{c}5.28- \\
\text { (COMe: 2.02) }\end{array}$ & 1.25 & 2.01 & 0.92 & 0.98 & & & & & $\mathrm{CDCL}_{3}$ & [70] \\
\hline $\begin{array}{l}\text { 3'-Acetyltrachelanthamine } \\
{\left[\mathrm{C}_{17} \mathrm{H}_{29} \mathrm{NO}_{5}\right]}\end{array}$ & 2.09 & $\begin{array}{l}\alpha 1.70 \\
\beta 2.02\end{array}$ & $\begin{array}{l}\alpha 3.28 \\
\beta 2.60\end{array}$ & $\begin{array}{l}\alpha 3.07 \\
\beta 2.65\end{array}$ & $\begin{array}{l}\alpha 1.89 \\
\beta 1.84\end{array}$ & $\begin{array}{l}\alpha 2.05 \\
\beta 1.63\end{array}$ & 3.38 & 4.16 & $\begin{array}{c}5.22- \\
\text { (COMe: 1.97) }\end{array}$ & 1.23 & 2.02 & 0.90 & 0.96 & & & & & $\mathrm{CDCL}_{3}$ & {$[128]$} \\
\hline $\begin{array}{l}\text { Canescine } \\
{\left[\mathrm{C}_{20} \mathrm{H}_{33} \mathrm{NO}_{7}\right]}\end{array}$ & & 5.83 & $\begin{array}{l}\alpha 3.95 \\
\beta 3.38\end{array}$ & $\begin{array}{l}\alpha 3.33 \\
\beta 2.65\end{array}$ & 2.09 & 5.38 & 4.35 & $\begin{array}{l}\alpha 4.79 \\
\beta 4.67\end{array}$ & 4.07 & 1.19 & 2.00 & 0.94 & 0.91 & 2.45 & & 1.26 & 1.26 & $\begin{array}{c}\mathrm{CDCL}_{3} / \\
\mathrm{D}_{6} \text {-DMSO }\end{array}$ & [154] \\
\hline $\begin{array}{l}\text { Canescenine } \\
{\left[\mathrm{C}_{20} \mathrm{H}_{33} \mathrm{NO}_{7}\right]}\end{array}$ & & 5.83 & $\begin{array}{l}\alpha 3.95 \\
\beta 3.38\end{array}$ & $\begin{array}{l}\alpha 3.33 \\
\beta 2.65\end{array}$ & 2.09 & 5.38 & 4.35 & $\begin{array}{l}\alpha 4.74 \\
\beta 4.73\end{array}$ & 3.89 & 1.19 & 2.00 & 0.94 & 0.91 & 2.45 & & 1.26 & 1.26 & $\begin{array}{c}\mathrm{CDCL}_{3} / \\
\mathrm{D}_{6} \text {-DMSO }\end{array}$ & [154] \\
\hline Cryptanthine & & 5.74 & $\begin{array}{l}\alpha 4.0 \\
\beta 3.37\end{array}$ & $\begin{array}{l}\alpha 3.40 \\
\beta 2.61\end{array}$ & 2.12 & 5.3 & 4.39 & $\begin{array}{l}\alpha 4.84 \\
\beta 4.64\end{array}$ & - & 6.1 & 1.98 & 1.89 & & 3.8 & 1.17 & 1.24 & & $\mathrm{CDCL}_{3}$ & {$[65]$} \\
\hline $\begin{array}{l}\text { 5'-Deoxylasiocarpine } \\
{\left[\mathrm{C}_{21} \mathrm{H}_{33} \mathrm{NO}_{6}\right]}\end{array}$ & & 5.8 & $\begin{array}{l}\alpha 3.50 \\
\beta 4.18\end{array}$ & $\begin{array}{l}\alpha 3.00 \\
\beta 3.40\end{array}$ & 1.8 & 5.12 & 4.37 & 4.88 & $\begin{array}{c}3.7 \text { - } \\
\text { (OMe: } 3.22)\end{array}$ & 1.21 & 2.20 & 0.95 & 0.94 & & 6.13 & 1.95 & 1.82 & $\mathrm{CDCL}_{3}$ & [123] \\
\hline $\begin{array}{l}\text { Echihumiline } \\
{\left[\mathrm{C}_{20} \mathrm{H}_{31} \mathrm{NO}_{7}\right]}\end{array}$ & & 5.87 & $\begin{array}{l}\alpha 3.35 \\
\beta 3.72\end{array}$ & $\begin{array}{l}\alpha 2.83 \\
\beta 2.83\end{array}$ & 2.09 & 5.48 & 4.13 & $\begin{array}{l}\alpha 4.65 \\
\beta 4.96\end{array}$ & 4.21 & 1.26 & - & 1.24 & 1.31 & 5.59 & - & 1.90 & 2.15 & $\mathrm{CDCL}_{3}$ & [91] \\
\hline $\begin{array}{l}\text { Echihumiline } N \text {-oxide } \\
{\left[\mathrm{C}_{20} \mathrm{H}_{31} \mathrm{NO}_{8}\right]}\end{array}$ & & 5.97 & $\begin{array}{l}\alpha 4.54 \\
\beta 4.77\end{array}$ & $\begin{array}{l}\alpha 3.73 \\
\beta 4.13\end{array}$ & $\begin{array}{l}\alpha 2.24 \\
\beta 2.89\end{array}$ & 5.77 & 5.45 & $\begin{array}{l}\alpha 4.71 \\
\beta 4.94\end{array}$ & 4.22 & 1.27 & - & 1.25 & 1.30 & 5.57 & - & 1.92 & 2.17 & $\mathrm{CDCL}_{3}$ & [91] \\
\hline $\begin{array}{l}\text { Echimidine isomer } \\
\text { (tigloyl) } \\
{\left[\mathrm{C}_{20} \mathrm{H}_{31} \mathrm{NO}_{7}\right]}\end{array}$ & & 5.81 & $\begin{array}{l}\alpha 3.34 \\
\beta 3.83\end{array}$ & $\begin{array}{l}\alpha 2.60 \\
\beta 3.27\end{array}$ & 2.05 & 5.37 & 4.32 & $\begin{array}{l}\alpha 4.60 \\
\beta 4.86\end{array}$ & 4.12 & 1.21 & & 1.17 & 1.26 & - & 6.05 & 1.91 & 1.76 & $\mathrm{CDCL}_{3}$ & [87]. \\
\hline
\end{tabular}


Table 5. Cont.

\begin{tabular}{|c|c|c|c|c|c|c|c|c|c|c|c|c|c|c|c|c|c|c|c|}
\hline Alkaloid & H-1 & $\mathrm{H}-2$ & $\mathbf{H}-3$ & H-5 & H-6 & $\mathrm{H}-7$ & $\mathrm{H}-8$ & H-9 & H-3' & $\mathbf{H}-4^{\prime}$ & H-5' & H-6' & C-7' & H-2" & H-3" & H-4" & H-5" & solvent & References \\
\hline \multicolumn{20}{|c|}{ Tertiary base and N-oxide alkaloids } \\
\hline $\begin{array}{l}\text { 7-Epi-echimiplatine N- } \\
\text { oxide } \\
{\left[\mathrm{C}_{15} \mathrm{H}_{25} \mathrm{NO}_{7}\right]}\end{array}$ & & 5.89 & $\begin{array}{l}\alpha 4.42 \\
\beta 4.21\end{array}$ & $\begin{array}{l}\alpha 3.74 \\
\beta 3.59\end{array}$ & $\begin{array}{l}\alpha 2.47 \\
\beta 2.01\end{array}$ & 4.67 & 4.55 & 4.78 & 4.18 & 1.14 & & 1.16 & 1.21 & & & & & $\mathrm{D}_{2} \mathrm{O}$ & {$[164]$} \\
\hline $\begin{array}{l}\text { Floridine } \\
{\left[\mathrm{C}_{17} \mathrm{H}_{29} \mathrm{NO}_{6}\right]}\end{array}$ & 2.23 & $\begin{array}{l}\alpha 2.15 \\
\beta 2.15\end{array}$ & $\begin{array}{l}\alpha 3.75 \\
\beta 2.77\end{array}$ & $\begin{array}{l}\alpha 3.48 \\
\beta 2.65\end{array}$ & 2.04 & $\begin{array}{l}\alpha 2.20 \\
\beta 1.70\end{array}$ & 4.01 & $\begin{array}{l}\alpha 4.16 \\
\beta 4.27\end{array}$ & $\begin{array}{c}5.45- \\
\text { (COMe: 1.98) }\end{array}$ & 1.36 & - & 1.25 & 1.40 & & & & & $\mathrm{CDCL}_{3}$ & {$[128]$} \\
\hline $\begin{array}{l}\text { Floridinine } \\
{\left[\mathrm{C}_{15} \mathrm{H}_{27} \mathrm{NO}_{5}\right]}\end{array}$ & 2.36 & $\begin{array}{l}\alpha 2.08 \\
\beta 2.20\end{array}$ & $\begin{array}{l}\alpha 3.51 \\
\beta 2.66\end{array}$ & $\begin{array}{l}\alpha 3.24 \\
\beta 2.85\end{array}$ & $\begin{array}{l}\alpha 2.0 \\
\beta 1.94\end{array}$ & $\begin{array}{l}\alpha 2.15 \\
\beta 1.63\end{array}$ & 3.70 & $\begin{array}{l}\alpha 4.20 \\
\beta 4.53\end{array}$ & 4.19 & 1.27 & - & 1.28 & 0.96 & & & & & $\mathrm{CDCL}_{3}$ & {$[128]$} \\
\hline $\begin{array}{l}\text { Floridimine } \\
{\left[\mathrm{C}_{15} \mathrm{H}_{27} \mathrm{NO}_{5}\right]}\end{array}$ & 2.30 & $\begin{array}{l}\alpha 2.17 \\
\beta 2.28\end{array}$ & $\begin{array}{l}\alpha 3.89 \\
\beta 2.83\end{array}$ & $\begin{array}{l}\alpha 3.59 \\
\beta 2.89\end{array}$ & 2.11 & $\begin{array}{l}\alpha 2.26 \\
\beta 1.80\end{array}$ & 4.30 & $\begin{array}{l}\alpha 4.35 \\
\beta 4.45\end{array}$ & 4.26 & 1.32 & - & 1.28 & 1.33 & & & & & $\mathrm{CDCL}_{3}$ & {$[128]$} \\
\hline $\begin{array}{l}\text { Helibracteatine } \\
{\left[\mathrm{C}_{13} \mathrm{H}_{21} \mathrm{NO}_{4}\right]}\end{array}$ & & 1.85 & $\begin{array}{l}\alpha 3.22 \\
\beta 2.54\end{array}$ & $\begin{array}{l}\alpha 3.22 \\
\beta 2.54\end{array}$ & $\begin{array}{l}\alpha 1.85 \\
\beta 2.05\end{array}$ & 4.08 & 3.30 & $\begin{array}{l}\alpha 4.35 \\
\beta 4.46\end{array}$ & 6.10 & 1.99 & 1.91 & & & & & & & $\begin{array}{c}\mathrm{CDCL}_{3}+ \\
\mathrm{D}_{2} \mathrm{O}\end{array}$ & {$[112]$} \\
\hline $\begin{array}{l}\text { Helibracteatinine } \\
{\left[\mathrm{C}_{13} \mathrm{H}_{21} \mathrm{NO}_{4}\right]}\end{array}$ & & $\begin{array}{l}\alpha 1.80 \\
\beta 2.10\end{array}$ & $\begin{array}{l}\alpha 3.35 \\
\beta 2.78\end{array}$ & $\begin{array}{l}\alpha 3.20 \\
\beta 2.60\end{array}$ & 1.80 & 5.09 & 3.30 & $\begin{array}{l}\alpha 3.64 \\
\beta 3.84\end{array}$ & & & & & & & 6.12 & 1.98 & 1.88 & $\begin{array}{c}\mathrm{CDCL}_{3}+ \\
\mathrm{D}_{2} \mathrm{O}\end{array}$ & {$[112]$} \\
\hline $\begin{array}{l}\text { Helindicine } \\
{\left[\mathrm{C}_{15} \mathrm{H}_{23} \mathrm{NO}_{4}\right]}\end{array}$ & & 5.94 & $\begin{array}{l}\alpha 4.40 \\
\beta 3.92\end{array}$ & $\begin{array}{l}\alpha 3.97 \\
\beta 3.34\end{array}$ & 2.15 & 4.63 & 4.90 & $\begin{array}{l}\alpha 4.98 \\
\beta 4.80\end{array}$ & 4.05 & 1.17 & 2.03 & 0.95 & 0.93 & & & & & $\mathrm{CD}_{3} \mathrm{OD}$ & {$[130]$} \\
\hline Heliotridine 2S-hydroxy- & & & & & & & & & & & & & & & & & & & \\
\hline $\begin{array}{l}\text { 2S-(1S-hydroxyethyl)-4- } \\
\text { methyl-pentanoyl ester }\end{array}$ & & 5.95 & $\begin{array}{l}3.70 \\
4.18\end{array}$ & $\begin{array}{l}3.10 \\
2.64\end{array}$ & 2.10 & 4.52 & 4.60 & 4.85 & $\begin{array}{l}1.74 \\
1.79\end{array}$ & 1.82 & 0.87 & 0.99 & $\begin{array}{c}3.78- \\
\left(8^{\prime}: 1.15\right)\end{array}$ & & & & & $\mathrm{CD}_{3} \mathrm{OD}$ & {$[50]$} \\
\hline$\left[\mathrm{C}_{16} \mathrm{H}_{27} \mathrm{NO}_{5}\right]$ & & & & & & & & & & & & & & & & & & & \\
\hline $\begin{array}{l}\text { Heliscabine } \\
{\left[\mathrm{C}_{13} \mathrm{H}_{21} \mathrm{NO}_{4}\right]}\end{array}$ & & 1.86 & $\begin{array}{l}\alpha 3.42 \\
\beta 3.05\end{array}$ & $\begin{array}{l}\alpha 3.30 \\
\beta 3.05\end{array}$ & 2.16 & 4.66 & 3.64 & $\begin{array}{l}\alpha 4.25 \\
\beta 4.35\end{array}$ & 6.10 & 1.99 & 1.89 & & & & & & & $\mathrm{CDCL}_{3}$ & [139] \\
\hline $\begin{array}{l}\text { Ilamine } \\
{\left[\mathrm{C}_{16} \mathrm{H}_{27} \mathrm{NO}_{5}\right]}\end{array}$ & & 5.81 & $\begin{array}{l}3.45 \\
4.10\end{array}$ & $\begin{array}{l}2.62 \\
3.35\end{array}$ & 1.86 & $\begin{array}{l}1.66 \\
2.10\end{array}$ & 4.43 & 4.78 & 3.81 & 1.28 & & 1.31 & $\begin{array}{c}1.20 \\
\text { (8-OMe: } 3.30)\end{array}$ & & & & & $\mathrm{CDCL}_{3}$ & {$[115]$} \\
\hline $\begin{array}{l}\text { Ilamine } \mathrm{N} \text {-oxide } \\
{\left[\mathrm{C}_{16} \mathrm{H}_{27} \mathrm{NO}_{6}\right]}\end{array}$ & & 5.81 & $\begin{array}{l}\alpha 3.43 \\
\beta 4.55\end{array}$ & $\begin{array}{l}\alpha 3.48 \\
\beta 3.79\end{array}$ & $\begin{array}{l}\alpha 1.99 \\
\beta 1.89\end{array}$ & 2.50 & 4.81 & $\begin{array}{l}\alpha 4.85 \\
\beta 4.69\end{array}$ & 3.80 & 1.26 & & 1.29 & $\begin{array}{c}1.22- \\
(8-\mathrm{OMe}: 3.30)\end{array}$ & & & & & $\mathrm{CDCL}_{3}$ & {$[115]$} \\
\hline
\end{tabular}


Table 5. Cont.

\begin{tabular}{|c|c|c|c|c|c|c|c|c|c|c|c|c|c|c|c|c|c|c|c|}
\hline Alkaloid & H-1 & $\mathbf{H}-2$ & H-3 & H-5 & H-6 & H-7 & H-8 & H-9 & H-3' & H-4' & H-5' & H-6' & C-7' & H-2" & H-3" & H-4" & H-5" & solvent & References \\
\hline \multicolumn{20}{|c|}{ Tertiary base and N-oxide alkaloids } \\
\hline $\begin{array}{l}\text { Isoechinatine }(9-(+)- \\
\text { Viridiflorylheliotridine) } \\
{\left[\mathrm{C}_{15} \mathrm{H}_{25} \mathrm{NO}_{5}\right]}\end{array}$ & & 5.66 & $\begin{array}{l}3.30 \\
3.83\end{array}$ & $\begin{array}{l}2.55 \\
3.22\end{array}$ & $\begin{array}{l}1.80 \\
1.92\end{array}$ & 4.13 & 3.97 & $\begin{array}{l}4.70 \\
5.00\end{array}$ & 3.92 & 1.24 & 2.13 & 0.89 & 0.85 & & & & & $\mathrm{CDCL}_{3}$ & {$[72,75]$} \\
\hline $\begin{array}{l}\text { Leptanthine } \\
{\left[\mathrm{C}_{15} \mathrm{H}_{25} \mathrm{NO}_{6}\right]}\end{array}$ & & 5.90 & $\begin{array}{l}\alpha 3.90 \\
\beta 4.40\end{array}$ & 3.90 & 2.20 & 4.65 & 4.95 & 4.90 & $\begin{array}{c}4.20-\mathrm{Me} \\
1.98\end{array}$ & 1.29 & 1.28 & 1.32 & & & & & & & {$[166]$} \\
\hline $\begin{array}{l}\text { Leptanthine N-oxide } \\
{\left[\mathrm{C}_{15} \mathrm{H}_{25} \mathrm{NO}_{7}\right]}\end{array}$ & & 6.05 & $\begin{array}{l}\alpha 4.65 \\
\beta 4.85\end{array}$ & $\begin{array}{l}4.1-4.0 \\
(\mathrm{~m})\end{array}$ & $\begin{array}{l}\alpha 2.23 \\
\beta 2.63\end{array}$ & 4.83 & 5.20 & $\begin{array}{l}\alpha 4.92 \\
\beta 4.98\end{array}$ & $\begin{array}{c}4.22-\mathrm{Me} \\
1.98\end{array}$ & 1.28 & 1.25 & 1.31 & & & & & & & {$[166]$} \\
\hline $\begin{array}{l}\text { Lactodine } \\
{\left[\mathrm{C}_{11} \mathrm{H}_{17} \mathrm{NO}_{4}\right]}\end{array}$ & & 5.68 & $\begin{array}{l}\alpha 3.90 \\
\beta 3.34\end{array}$ & $\begin{array}{l}\alpha 3.26 \\
\beta 2.59\end{array}$ & $\begin{array}{l}\alpha 1.93 \\
\beta 1.87\end{array}$ & 4.10 & 4.02 & $\begin{array}{l}\alpha 5.01 \\
\beta 4.89\end{array}$ & 4.20 & 1.39 & & & & & & & & $\begin{array}{c}\mathrm{CDCL}_{3}+ \\
\mathrm{D}_{2} \mathrm{O}\end{array}$ & {$[187]$} \\
\hline $\begin{array}{l}\text { Lithosenine } \\
{\left[\mathrm{C}_{20} \mathrm{H}_{33} \mathrm{NO}_{8}\right]}\end{array}$ & & 5.78 & $\begin{array}{l}\alpha 3.77 \\
\beta 3.29\end{array}$ & $\begin{array}{l}\alpha 3.16 \\
\beta 2.57\end{array}$ & 1.96 & 5.21 & 4.20 & $\begin{array}{l}\alpha 4.66 \\
\beta 4.52\end{array}$ & 4.03 & 1.13 & & 1.17 & 1.11 & 2.33 & & 1.16 & 1.16 & $\mathrm{CDCL}_{3}$ & {$[156]$} \\
\hline $\begin{array}{l}\text { Megalanthonine } \\
{\left[\mathrm{C}_{15} \mathrm{H}_{27} \mathrm{NO}_{5}\right]}\end{array}$ & 2.78 & $\begin{array}{l}\alpha 1.89 \\
\beta 2.15\end{array}$ & $\begin{array}{l}\alpha 3.26 \\
\beta 2.61\end{array}$ & $\begin{array}{l}\alpha 3.21 \\
\beta 2.73\end{array}$ & $\begin{array}{l}\alpha 2.03 \\
\beta 2.14\end{array}$ & 4.29 & 3.48 & $\begin{array}{l}\alpha 4.09 \\
\beta 4.46\end{array}$ & 2.64 & - & 2.64 & 3.70 & 1.34 & & 6.80 & 1.96 & 1.80 & $\mathrm{CDCL}_{3}$ & [134] \\
\hline $\begin{array}{l}\text { Methylechiuplatine } \\
{\left[\mathrm{C}_{20} \mathrm{H}_{29} \mathrm{NO}_{7}\right]}\end{array}$ & & 5.77 & $\begin{array}{l}\alpha 3.97 \\
\beta 3.37\end{array}$ & $\begin{array}{l}\alpha 3.32 \\
\beta 2.64\end{array}$ & 2.09 & 5.40 & 4.33 & $\begin{array}{l}\alpha 4.68 \\
\beta 4.62\end{array}$ & & & & & & & & & & & {$[65]$.} \\
\hline $\begin{array}{l}\text { Neo coramandaline } \\
{\left[\mathrm{C}_{15} \mathrm{H}_{27} \mathrm{NO}_{4}\right]}\end{array}$ & 1.9 & $\begin{array}{l}\alpha 1.85 \\
\beta 1.55\end{array}$ & $\begin{array}{l}\alpha 3.20 \\
\beta 2.50\end{array}$ & $\begin{array}{l}\alpha 3.05 \\
\beta 2.64\end{array}$ & $\begin{array}{l}\alpha 1.85 \\
\beta 1.55\end{array}$ & $\begin{array}{l}\alpha 1.90 \\
\beta 1.35\end{array}$ & 3.56 & $\begin{array}{l}\alpha 4.30 \\
\beta 4.20\end{array}$ & 3.98 & 1.23 & 2.14 & 0.88 & 0.92 & & & & & $\mathrm{CDCL}_{3}$ & [74] \\
\hline $\begin{array}{l}\text { Onosmerectine N-oxide } \\
{\left[\mathrm{C}_{15} \mathrm{H}_{25} \mathrm{NO}_{7}\right]}\end{array}$ & & 5.96 & $\begin{array}{l}\alpha 4.66 \\
\beta 4.45\end{array}$ & 3.87 & $\begin{array}{l}\alpha 2.58 \\
\beta 2.12\end{array}$ & 4.75 & 4.85 & $\begin{array}{l}\alpha 4.92 \\
\beta 4.88\end{array}$ & & 4.22 & 1.26 & 1.31 & 1.27 & & & & & $\mathrm{CD}_{3} \mathrm{O}$ & [164] \\
\hline $\begin{array}{l}\text { (7S, 8R)-Petranine } \\
{\left[\mathrm{C}_{14} \mathrm{H}_{20} \mathrm{CINO}_{3}\right]}\end{array}$ & & 5.82 & $\begin{array}{l}\alpha 4.55 \\
\beta 4.77\end{array}$ & $\begin{array}{l}\alpha 4.09 \\
\beta 4.35\end{array}$ & $\begin{array}{l}\alpha 2.48 \\
\beta 2.70\end{array}$ & 4.92 & 5.36 & 4.78 & 6.18 & 2.00 & 1.90 & & & $\begin{array}{l}\alpha 5.42 \\
\beta 5.75\end{array}$ & & & & $\mathrm{CDCL}_{3}$ & [89] \\
\hline $\begin{array}{l}\text { (7S, } 8 \mathrm{~S} \text { )-Petranine } \\
{\left[\mathrm{C}_{14} \mathrm{H}_{20} \mathrm{CINO}_{3}\right]}\end{array}$ & & 5.83 & $\begin{array}{l}\alpha 4.54 \\
\beta 4.78 \\
\end{array}$ & $\begin{array}{l}\alpha 4.09 \\
\beta 4.35 \\
\end{array}$ & $\begin{array}{l}\alpha 2.51 \\
\beta 2.69 \\
\end{array}$ & 4.94 & 5.38 & 4.88 & 6.20 & 2.02 & 1.90 & & & $\begin{array}{l}\alpha 5.71 \\
\beta 5.39 \\
\end{array}$ & & & & $\mathrm{CDCL}_{3}$ & [89] \\
\hline
\end{tabular}


Table 5. Cont.

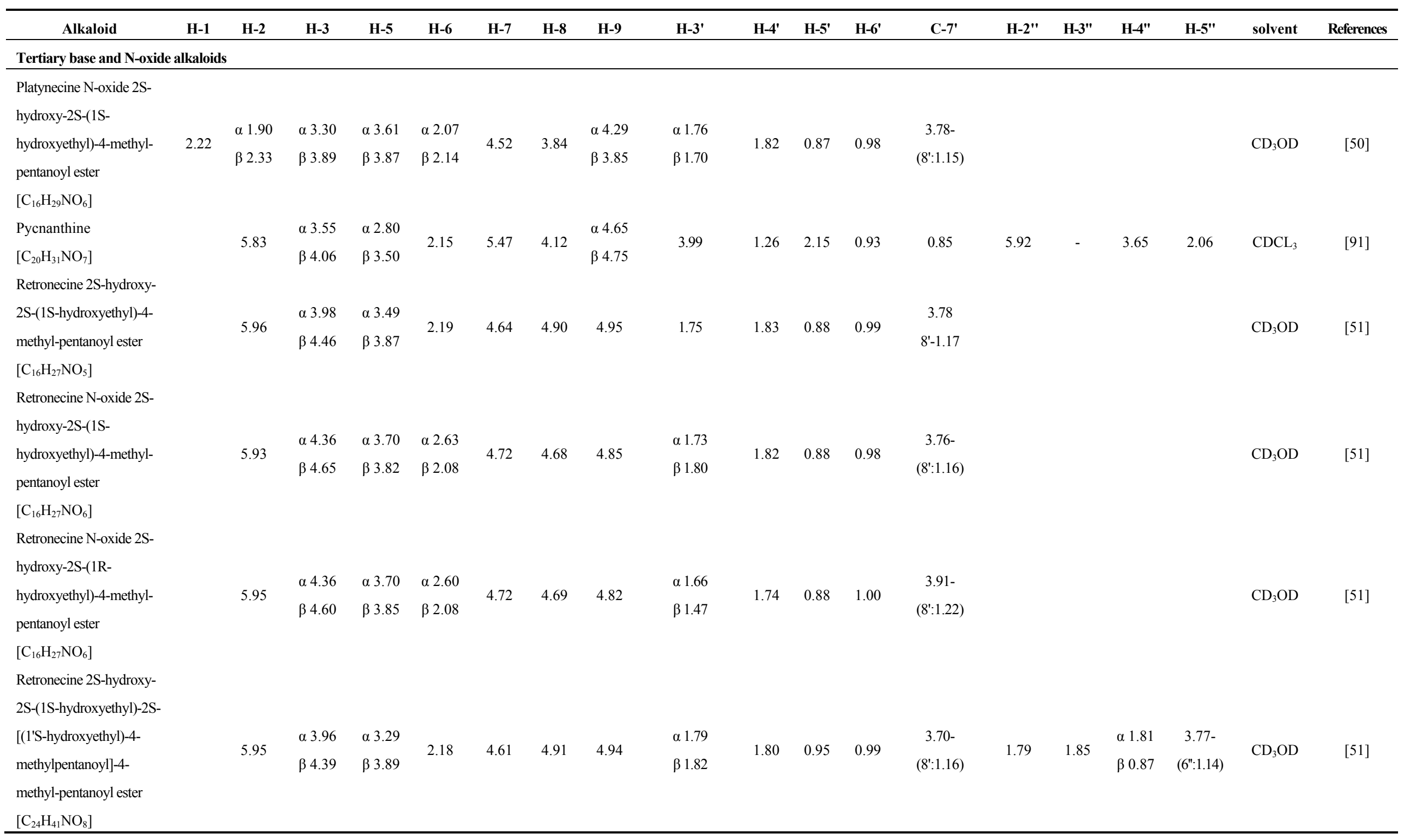


Table 5. Cont.

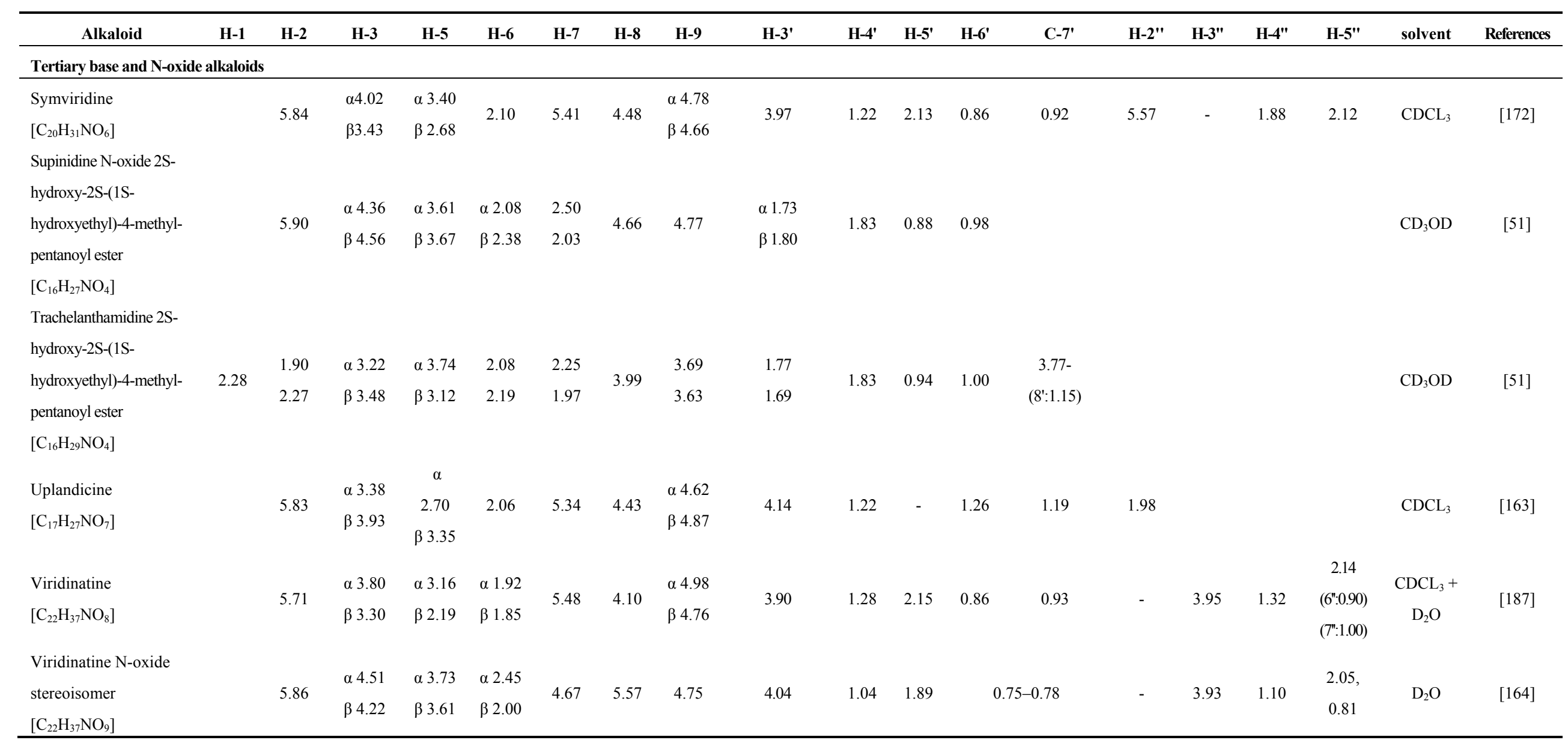




\section{Biological Activity of PAs}

\subsection{Antimicrobial Activity}

The growth of bacterial species, mostly human pathogens such as E. coli, S. pneumoniae, Bacillus subtilis, B. anthracis and Staphylococcus aureus were inhibited by different pure PAs and PA extracts [90,110]. Successive root bark extracts of Cordia gilletii (Boraginaceae) were tested for their antimicrobial activity against 10 strains of bacteria and 1 strain of fungi by broth microdilution and agar diffusion methods. The methanol extract showed direct antimicrobial activity against all tested microorganisms with minimum inhibitory concentrations (MIC) ranging between 125 and 1,000 $\mu \mathrm{g} / \mathrm{ml}$. [223]. Joosten and van Veen [224] discussed the impact of plant-produced PAs on plantassociated microorganisms, their detoxification by microorganisms and the ecological consequences of this activity.

\subsection{Sequestration in Insects and Antifeedant Properties}

Several insects are PA specialists which not only tolerate PAs of their host plants but use them for their own chemical defence against predators, as morphogens and even as pheromones [101,144,225-231]. Defensive PAs can be obtained as larvae from food plants and as adults via nectar. PA sequestering insects often show aposematic colouration. Several moths and butterflies use unsaturated PAs to produce dihydropyrrolizidines with pheromone activity [110]. In some moths PAs are morphogens inducing the formation of large coremata in males, which function as pheromone emitting organs. A recent review published by Macel [232], threw light on the dual role PAs in plant-insect interactions.

The antifeedant activity of six PAs isolated from Anchusa strigosa towards the generalist herbivore Spodoptera exigua and the cabbage specialist Pieris brassicae was evaluated [50]. 1,2-Unsaturated PAs (e.g., retronecine 2S-hydroxy (1S-hydroxyethyl)-4-methyl-pentanoyl ester and its N-oxide) reduced feeding by $P$. brassicae by 52 and $68 \%$, respectively compared to control. However, the alkaloids lacking to 1,2-double bond (trachelanthamidine ester and platynecine ester) showed no deterrent activity.

\subsection{Biological Importance}

Toxicity of 1,2-unsaturated PAs as hepatotoxic, pulmotoxic, hemolytic, antimitotic, teratogenic, mutagenic and carcinogenic natural products for humans and livestock is well documented [23-32,35,36]. The potential PA contamination of food and feeding stuff has attracted recurrent attention. It is evident, that humans should not ingest food or herbal teas that contain PAs. However, some saturated PAs have interesting pharmacological and biological effects, e.g., spasmolytic, antihistaminic, anti-HIV and antiviral activities and as glucosidase inhibitor [233-235]. Solanecio angulatus and Crotalaria phillipsiae (PA containing plants from the families Asteraceae and Fabaceae) serve as sources of novel trypanocidal compounds [236]. The crude ethanol and hexane extracts of Heliotropium subulatum showed significant antiviral activity to Coxsackie, Poliomyelitis and Measles at 500 and $100 \mu \mathrm{g} / \mathrm{mL}$, respectively, while heliotrine and 7-angeloylheliotrine demonstrated activity against Poliomyelitis and Vesicular stomatitis at concentration of $10 \mu \mathrm{g} / \mathrm{mL}$ [237]. PAs can inhibit the specific binding of 
radioligands to muscarinic acetylcholine and serotonin receptors and to a lesser degree to adrenergic receptors [20]. This neuromodulatory effect might be responsible for the avoidance of PA plants by many herbivores.

\section{Chemotaxonomic Information of PAs in Boraginaceae}

The distribution of PAs is documented in Tables 1 and 2. A comparison of phytochemical occurrences of alkaloids with regard to recent results in the molecular phylogeny of the Boraginaceae was not intended in this review, but will be part of a separate publication. It has been shown previously that the occurrence of PAs in the plant kingdom does not necessarily mirror phylogenetic relatedness because the genes which encode the enzymes of alkaloid biosynthesis can be switched on or off [2,238].

\subsection{Genus Amsinckia}

PA profiles of twenty species and varieties of the genus Amsinckia have been investigated. All identified alkaloids were from retronecine, (+)- and (-)-supinidine, (+)-isoretrenocanol and trachelanthamidine types [42-44,46,104].

\subsection{Genus Cynoglossum}

Plants belongs to this genus contain alkaloids mainly derived from heliotridine type, in addition to $(+)$ - and (-)-supinidine and trachelanthamidine bases [60,67-78,80-86,239].

\subsection{Genus Echium}

PAs of about 15 species from this genus have been investigated which accumulate PAs from retronecine type [11,28,29,60,87,89-93,96,99]. However, in earlier papers [47,97,98], the structure of major compounds in Echium vulgare were wrongly assigned to heliosupine and 3'-acetylheliosupine by means of paper chromatography and/or mass spectrometry. These compounds belong to heliotridine types. Recent publications revised the structures to the stereoisomeric analogue, echimidine and 3'-acetylechimidine (retronecine type), based on NMR and other advanced spectroscopic techniques [11,96,229].

\subsection{Genus Heliotropium}

Biosynthetic studies on necines that have been incorporated into PAs as well as the isolated and/or identified alkaloids in the genus Heliotropium indicated that it contains retronecine, $(-)$-trachelanthamidine, (-)-supindine, (-)-isoretronecanol, laburnine, lindelofidine, platynecine and subulacine nucleus, in addition heliotridine type PAs [28,29]. In addition to the isolation of the open-chained necines, a cyclic diester PA, e.g., helindicine has been recorded in H. indicum [130], incanine in $H$. olgae and trichodesmine in H. arguzioides [29].

\subsection{Genus Paracaryum and Paracynoglossum}

Only PAs from the heliotridine and (-)-trachelanthamidine type have been reported to be accumulated in these species $[28,29,40,151]$. 


\subsection{Genus Rindera}

All the previously examined Rindera species contain heliotridine-type PAs [120,152,169]. Echinatine is considered as a chemotaxonomical marker in this genus.

\subsection{Genus Symphytum}

Eleven Symphytum species ( $S$. aintabicum, S. bohemium, S. consolidum, S. grandiflorum, S. ibericum, S. orientale, S. peregrinum, S. sylvaticum subsp. sepulcrale var. sepulcrale, S. tanaiense, S. tuberosum, $S . \times$ uplandicum) have been documented to synthesis pyrrolizidine alkaloids of retronecine type $[17,29,170,177]$. However, both retronecine and heliotridine type alkaloids were identified in S. asperum [171], S. caucasium [174], and S. officinale [29].

\subsection{Other Boraginaceae Species}

Species of genus Alkanna [40,41,184], Cryptantha [63-66], Mertensia [157], and Messerschmidia [158] produce only PAs of the retronecine type. Whereas Onosma heterophyllum and O. erecta [164] accumulate only heliotridine type PAs, other species of this genus synthesise retronecine type PAs $[60,161,163,166,167]$ in addition to, supinidine [163] and trachelanthamidine type [167].

\section{Conclusions}

Our knowledge on PAs in Boraginaceae has improved substantially during the last decades, especially as advanced analytical methods, such as GLC, HPLC in combination with mass spectrometry have been widely applied. However, information on the biochemistry and physiology of PAs in plants and their function in plant insect interactions is still limited. More research has addressed pharmacological and toxicological properties of PAs in food plants and herbal medicines; public awareness is rightly concerned with PAs in food items and their place in the food chain. This review summarizes the state of our existing knowledge.

\section{Acknowledgments}

The authors thank Sameh Soliman and Ahmed El-Kadeem, Department of Pharmacognosy, Faculty of Pharmacy, Zagazig University for providing some published papers.

\section{Author Contributions}

This review was planned by both authors. Assem El-Shazly collected the information and drafted the manuscript which was interpreted and edited by Michael Wink. Both authors finally approved the published version.

\section{Conflicts of Interest}

Michael Wink is Editor-in-Chief of Diversity. 


\section{References}

1. Wink, M. Plant breeding: Importance of plant secondary metabolites for protection against pathogens and herbivores. Theor. Appl. Genet. 1988, 75, 225-233.

2. Wink, M. Evolution of secondary metabolites from an ecological and molecular phylogenetic perspective. Phytochemistry 2003, 64, 3-19.

3. Detzel, A.; Wink, M. Attraction, deterrence or intoxication of bees (Apis mellifera) by plant allelochemicals. Chemoecology 1993, 4, 8-18.

4. Culvenor, C.C.J.; Edgar, J.A.; Smith, L.W. Pyrrolizidine alkaloids in Honey from Echium plantagineum L. J. Agric. Food Chem. 1981, 29, 958-960.

5. Hartmann, T. Chemical ecology of pyrrolizidine alkaloids. Planta 1999, 207, 483-495.

6. Prakash, A.S.; Pereira, T.N.; Reilly, P.E.A.; Seawright, A.A. Pyrrolizidine alkaloids in human diet. Mutation Res. 1999, 443, 53-67.

7. Edgar, J.A.; Smith, L.W. Transfer of Pyrrolizidine Alkaloids into Eggs: Food Safety Implications. ACS Symp. Ser. 1999, 754, 118-128.

8. Edgar, J.A.; Roeder, E.; Molyneux, R.J. Honey from plants containing pyrrolizidine alkaloids: A potential threat to health. J. Agric. Food Chem. 2002, 50, 2719-2730.

9. Fu, P.P.; Yang, Y.; Xia, Q.; Chou, M.W.; Cui, Y.Y.; Lin, A.G. Pyrrolizidine alkaloids-tumorigenic components in Chinese herbal medicines and dietary supplements. J. Food Drug Anal. 2002, 10, $198-211$.

10. Azadbakht, M.; Talavaki, M. Qualitative and quantitative determination of pyrrolizidine alkaloids of wheat and flour contaminated with Senecio in Mazandaran province farm. Int. J. Pharm. Res. 2003, 2, 179-183.

11. Boppré, M.; Colegate, S.M.; Edgar, J.A. Pyrrolizidine alkaloids of Echium vulgare honey found in pure pollen. J. Agric. Food Chem. 2005, 53, 594-600.

12. Boppré, M.; Colegate, S.M.; Edgar, J.A.; Fischer, W.O. Hepatotoxic pyrrolizidine alkaloids in pollen and drying-related implication for commercial processing of bee pollen. J. Agric. Food Chem. 2008, 56, 5662-5672.

13. Sharma, R.A.; Singh, B.; Singh, D.; Chandrawat, P. Ethnomedicinal, pharmacological properties and chemistry of some medicinal plants of Borginaceae in India. J. Med. Plant Res. 2009, 3, 1153-1175.

14. European Food Safety Authority. Opinion of the scientific panel on contamination in the food chain on a request from the European Commission related to pyrrolizidine alkaloids as undesirable substances in animal feed. EFSA J. 2007, 447, 1-51.

15. Kakar, F.; Akbarian, Z.; Leslie, T.; Mustafa, M.L.; Watson, J.; van Egmond, H.P.; Omar, M.F.; Mofleh, J. An outbreak of hepatic veno-occlusive disease in western Afghanistan associated with exposure to wheat flour contaminated with pyrrolizidine alkaloids. J. Toxicol. 2010, doi:10.1155/2010/313280.

16. Kempf, M.; Heil, S.; Haßlauer, I.; Schmidt, L.; von der Ohe, K.; Theuring, C.; Reinhard, A.; Schreier, P.; Beuerle, T. Pyrrolizidine alkaloids in pollen and pollen products. Mol. Nutr. Food Res. 2010, 54, 292-300.

17. Staiger, C. Comfrey: A Clinical Overview. Phytother. Res. 2012, 26, 1441-1448. 
18. Cooper, R.A.; Huxtable, R.J. The relationship between reactivity of metabolites of pyrrolizidine alkaloids and extrahepatic toxicity. Proc. West Pharmacol. Soc. 1999, 42, 13-16.

19. Kim, H.Y.; Stermitz, F.R.; Li, J.K.; Coulombe, R.A. Comparative DNA cross-linkage by activated pyrrolizidine alkaloids. Food Chem. Toxicol. 1999, 37, 619-625.

20. Schmeller, T.; El-Shazly, A.; Wink, M. Allelochemical activities of pyrrolizidine alkaloids: Interaction with neuroreceptors and acetylcholine related enzymes. J. Chem. Ecol. 1997, 23, $399-416$.

21. Wink, M.; Roberts, M.F. Compartmentation of alkaloid synthesis, transport and storage. In Alkaloids, Biochemistry, Ecology and Medicinal Application; Roberts, M.F., Wink, M., Eds.;. Plenum Press: New York, NY, USA and London, UK, 1998; Chapter 10, pp. 239-269.

22. Wink, M.; Schmeller, T.; Latz-Brüning, B. Modes of action of allelochemical alkaloids: Interaction with neuroreceptors, DNA and other molecular targets. J. Chem. Ecol. 1998, 24, 1881-1937.

23. Bull, L.B.; Culvenor, C.C.J.; Dick, A.T. The Pyrrolizidine Alkaloids; North Holland: Amsterdam, The Netherlands, 1968.

24. McLean, E.K. The toxic activities of pyrrolizidine (Senecio) alkaloids. Pharmacol. Rev. 1970, 22, 429-476.

25. Smith, L.W.; Culvenor, C.C.J. Plant sources of hepatotoxic pyrrolizidine alkaloids. J. Nat. Prod. 1981, 44, 129-152.

26. Robins, D.J. The pyrrolizidine alkaloids. Fortschr. Chem. Org. Naturst. 1982, 41, 115-203.

27. Mattocks, A.R. Chemistry and Toxicology of Pyrrolizidine Alkaloids; Academic Press: London, UK and New York, NY, USA, 1986.

28. Rizk, A.M. Naturally Occurring Pyrrolizidine Alkaloids; CRC Press: Boca Raton, FL, USA, 1991.

29. Hartmann, T.; Witte, L. Chemistry, biology and chemoecology of pyrrolizidine alkaloids. In Alkaloids: Chemical and Biological Perspectives; Pelletier, S.W., Ed.; Elsevier Science Ltd.: Kidlington, UK, 1995; Vol. 9, Charpter 4, pp. 155-233.

30. Roeder, E. Medicinal plants in Europe containing pyrrolizidine alkaloids. Pharmazie 1995, 50, 83-98.

31. Roeder, E. Analysis of pyrrolizidine alkaloids. Curr. Org. Chem. 1999, 3, 557-576.

32. Roeder, E. Medicinal plants in China containing pyrrolizidine alkaloids. Pharmazie 2000, 55, 711-725.

33. Wink, M. Plant secondary metabolism: Diversity, function and its evolution. Nat Prod. Commun. 2008, 3, 1205-1216.

34. Wink, M.; van Wyk, B.E. Mind-altering and Poisonous Plants of the World; Briza Publications: Pretoria, South Africa, 2008.

35. Roeder, E.; Wiedenfeld, H. Pyrrolizidine alkaloids in medicinal plants of Mongolia, Nepal and Tibet. Pharmazie 2009, 64, 699-716.

36. Wiedenfeld, H. Plants containing pyrrolizidine alkaloids: Toxicity and problems. Food Addit. Contam. 2011, 28, 282-292.

37. Jiang, Y.; Fu, P.P.; Lin, G. Hepatotoxicity of naturally occurring pyrrolizidine alkaloids. Asian J. Pharmacodyn. Pharmacokinet. 2006, 6, 187-192. 
38. Dreger, M.; Stanisławska, M.; Krajewska-Patan, A.; Mielcarek, S.; Mikołajczak, L.P.; Buchwald, W. Pyrrolizidine alkaloids-chemistry, biosynthesis, pathway, toxicity, safety and perspectives of medicinal usage. Herba Polonica 2009, 55, 127-147.

39. Roeder, E.; Wiedenfeld, H. Plants containing pyrrolizidine alkaloids used in the Traditional Indian Medicine-including Ayurveda. Pharmazie 2013, 68, 83-92.

40. El-Shazly, A.; El-Domiaty, M.; Witte, L.; Wink, M. Pyrrolizidine alkaloids in members of the Boraginaceae from Sinia (Egypt). Biochem. Syst. Ecol. 1998, 26, 619-639.

41. Roeder, E.; Sarg, T.; El-Dahmy, S.; Abdel-Ghani, A. Pyrrolizidine alkaloids from Alkanna orientalis. Fitoterapia 1992, 63, 405-408.

42. Kelley, R.B.; Seiber, J.N. Pyrrolizidine alkaloid chemosystematics in Amsinckia. Phytochemistry 1992, 31, 2369-2387.

43. Kelley, R.B.; Seiber, J.N. Pyrrolizidine alkaloid from Amsinckia. Phytochemistry 1992, 31, 2513-2518.

44. Culvenor, C.C.J.; Smith, L.W. Alkaloids of Amsinckia species: A. intermedia, A. hispida and A. lycoposoides. Aust. J. Chem. 1966, 19, 1955-1964.

45. Roitman, J.N. Pyrrolizidine alkaloids of Amsinckia menziesii. Aust. J. Chem. 1983, 36, 769-778.

46. Cooper, R.A.; Bowers, R.J.; Beckham, C.J.; Huxtable, R.J. Preparative separation of pyrrolizidine alkaloids by high-speed counter-current chromatography. J. Chromatogr. A 1996, 732, 43-50.

47. Pedersen, E. Pyrrolizidine alkaloids in Danish species of the family Boraginaceae. Arch. Pharm. Chem. Sci. Ed. 1975, 3, 55-64.

48. Broch-Due, A.I.; Aasen, A.J. Alkaloids of Anchusa officinalis L. Identification of pyrrolizidine alkaloid lycopsamine. Acta Chem. Scand. Ser. B. 1980, 34, 75-77.

49. Hendriks, H.; Bruins, A.P.; Huizing, H.J. Detection of curassavine and some related pyrrolizidine alkaloids in an Anchusa officinalis strain with means of positive ion and negative ion chemical ionization GC-MS. Biomed. Environ. Mass Spctrom. 1988, 17, 129-132.

50. Siciliano, T.; de Leo, M.; Bader, A.; de Tommasi, N.; Vrieling, K.; Braca, A.; Morelli, I. Pyrrolizidine alkaloids from Anchusa strigosa and their antifeedant activity. Phytochemistry 2005, 66, 1593-1600.

51. Braca, A.; Bader, A.; Siciliano, T.; Morelli, I.; Tommasi, N. New pyrrolizidine alkaloid and glycosides from Anchusa strigosa. Planta Med. 2003, 69, 835-841.

52. El-Dahmy, S.; Adel Ghani, A. Alkaloids of Arnebia decumbens Vent. Az. J. Pharm. Sci. 1995, 15, 24-34.

53. Roeder, E.; Rengel-Mayer, B. Pyrrolizidine alkaloids from Arnebia euchroma. Planta Med. 1993, 59, 192.

54. Wassel, G.; El-Menshawi, B.; Saeed, A.; Mahran, G. Toxic pyrrolizidine alkaloids of certain Boraginaceae. Acta Pharm. Suec. 1987, 24, 199-204.

55. Langer, T.; Franz, C. Determination of pyrrolizidine alkaloids in commercial samples of borage seed oil products by GC-MS. Sci. Pharm. 1997, 65, 321-328.

56. Herrmann, M.; Joppe, H.; Schmaus, G. Thesinine-4'-O- $\beta$-D-glucoside the first glycosylated plant pyrrolizidine alkaloid from Borago officinalis. Phytochemistry 2002, 60, 399-402. 
57. Telezhenetskaya, M.V.; Matkarmov, A.D.; Khadzhibekov, S.N.; Yunusov, S.Y. Alkaloids of some Central Asian plants. Khim. Prir. Soedin 1987, 3, 663-664.

58. Siddiqi, M.A.; Suri, K.A.; Suri, O.P.; Atal, C.K. A new pyrrolizidine alkaloid from Caccinia glauca. Pytochemistry 1978, 17, 2049-2050.

59. El-Shazly, A. Pyrrolizidine alkaloids of Cerinthe glabra Miller (Boraginaceae). Zagazig $J$. Pharm. Sci. 2004, 13, 1-5.

60. Mroczek, T.; Baj, S.; Chrobok, A.; Glowniak, K. Screening for pyrrolizidine alkaloids in plant materials by electron ionization RP-HPLC-MS with thermabeam interface. Bio. Med. Chromatogr. 2004, 18, 745-751.

61. Roeder, E.; Wiedenfeld, H.; Kaus, K.J. Das Pyrrolizidinalkaloid Intermedin aus Cerinthe minor L. Sci. Pharm. 1990, 58, 9-13. (In German)

62. Wassel, G.; El-Menshawi, B.; Saeed, A.; Mahran, G.; Reisch, J. New sources of pyrrolizidine alkaloids: Genus Cordia (Ehretiaceae) and Schismus (Gramineae). Sci. Pharm. 1987, 55, 163-166.

63. Stermiz, F.; Pass, M.A.; Kelley, R.B.; Liddell, J.R. Pyrrolizidine alkaloids from Cryptantha species. Phytochemistry 1993, 33, 383-387.

64. Williams, M.T.; Warnock, B.J.; Betz, J.M.; Beck, J.J.; Gardner, D.R.; Lee, S.T.; Molyneux, R.J.; Colegate, S.M. Detection of high levels of pyrrolizidine-N-oxides in the endangered plant Cryptantha crassipes (Terlingua creek cat's-eye) using HPLC-ESI-MS. Phytochem. Anal. 2011, 22, 532-540.

65. Colegate, S.M.; Gardner, D.R.; Davis, T.Z.; Betz, J.M.; Panter, K.E. Dehydropyrrolizidine alkaloids in two Cryptantha species: Including two new open chain diesters one of which is amphoteric. Phytochem. Anal. 2013, 24, 201-212.

66. Beck, J.J.; Stermitz, F.R. Pyrrolizidine alkaloids from Brickellia grandiflora and Cryptantha jamesii. Biochem. Syst. Ecol. 2002, 30, 1079-1081.

67. El-Shazly, A.; Sarg, T.; Ateya, A.; Abdel-Aziz, E.; Witte, L.; Wink, M. Pyrrolizidine alkaloids of Cynoglossum officinale and Cynoglossum amabile (Family Boraginaceae). Biochem. Syst. Ecol. 1996, 24, 415-421.

68. Culvenor, C.C.J.; Smith, L.W. Alkaloids of Cynoglossum australe and C. amabile. Aust. J. Chem. 1967, 20, 2499-2503.

69. Damianakos, H.; Jeziorek, M.; Pietrosiuk, A.; Sykłowska-Baranek, K.; Chinou, I. Isolation of pyrrolizidine alkaloids from Cynoglossum columnae Ten. (Boraginaceae). Planta Med. 2013, doi:10.1055/s-0033-1352119.

70. El-Shazly, A.; Sarg, T.; Witte, L.; Wink, M. Pyrrolizidine alkaloids from Cynoglossum creticum. Phytochemistry 1996, 42, 1217-1221.

71. Zalkow, L.H.; Bonetii, S.; Gelbaum, L.T.; Gordon, M.M.; Patil, B.B.; Shani, A.; van Derveer, I.D. Pyrrolizidine alkaloid from middle eastern plans. J. Nat. Prod. 1979, 42, 603-614.

72. Asibal, C.F.; Glinski, J.A.; Gelbaum, L.T.; Zalkow, L.H. Pyrrolizidine alkaloids from Cynoglossum creticum. Synthesis of pyrrolizidine alkaloids echinatine, rinderine and analogues. J. Nat. Prod. 1989, 52, 109-118.

73. Chen, Z.W.; Tang, J.; Li, J.; Zhang, Q.; Zhao, X.; Hu, J. Constituents of Cynoglossum zeylanicum. Zhongguo Yaoke Daxue Xuebao (J. Chin. Pharm.) 1987, 18, 51-53. (In Chinese) 
74. Ravi, S.; Lakshmanan, A.J. Coromandaline, a pyrrolizidine alkaloid from Cynoglossum furcatum. Ind. J. Chem. 2000, 39B, 80-82.

75. Ravicumar, R.; Lakshmanan, A.J. Isoechinatine, a pyrrolizidine alkaloid from Cynoglossum furcatum. Ind. J. Chem. 2004, 43B, 406-409.

76. Ravi, S.; Ravikumar, R.; Lakshmanan, A.J. Pyrrolizidine alkaloids from Cynoglossum furcatum. J. Asian Nat. Prod. Res. 2008, 10, 307-310.

77. Mattoks, A.R.; Pigott, C.D. Pyrrolizidine alkaloids from Cynoglossum germanicum. Phytochemistry 1990, 29, 2871-2872.

78. Suri, K.A.; Sawhney, R.S.; Atal, C.K. Pyrrolizidine alkaloids from Cynoglossum lanceolatum, C. glochidiatum and Lindelofia angustifolia. Indian J. Pharm. 1975, 37, 69-70.

79. Crowely, H.C.; Culvenor, C.C.J. Alkaloids of Cynoglossum latifolium, latifoline and 7-angeloylretronecine. Aust. J. Chem. 1962, 15, 139-144.

80. Kelly, H.A.; Robins, D.J. Pyrrolizidine alkaloids from Cynoglossum macrostylum. Fitoterapia 1992, 63, 91.

81. Guner, N. Pyrrolizidine alkaloids from Cynoglossum montanum. Marmara Univ. Eczacilik Derg. 1987, 3, 57-60.

82. Hagan, D.; Robins, D.J. Pyrrolizidine alkaloids from Cynoglossum nervosum. Fitoterapia 1991, 62, 186-187.

83. Resch, J.F.; Meinwald, J. A revised structure for acetylheliosupine. Pytochemistry 1982, 21, 2430-2431.

84. Van Dam, N.M.; Witte, L.; Theuring, C.; Hartmann, T. Distribution, biosynthesis and turnover of pyrrolizidine alkaloids in Cynoglossum officinale. Phyochemistry 1995, 39, 287-292.

85. Man'ko, I.V.; Marchenko, L.G. Pictumine, a new alkaloid from Cynoglossum pictum. Khim. Prir. Soedin. 1972, 5, 655-656.

86. Man'ko, I.V. Alkaloids of Cynoglossum amabile and C. viridiflorum. Rastit. Resur. 1972, 8, 243-246.

87. Mehrabani, M.; Ghannadi, A.; Sajjadi, E.; Ghassemi, N.; Shams-Ardakani, M. Toxic pyrrolizidine alkaloids of Echium amoenum Fisch. \& Mey. DARU J. Pharm. Sci. 2006, 14, 122-127.

88. Sarg, T.; El-Dahmy, S.; Abdel-Aziz, E.; Abdel-Ghani, A.; Roeder, E. Pyrrolizidine alkaloids from Echium angustifolium. Fitoterapia 1992, 63, 466-468.

89. Alali, F.Q.; Tahboub, Y.R.; Ibrahim, E.S.; Qandil, A.; Tawaha, K.; Burgess, J.P; Sy, A.; Nakanishi, Y.; Kroll, D.J.; Oberlies, N.H. Pyrrolizidine alkaloids from Echium glomeratum (Boraginaceae). Phytochemistry 2008, 69, 2341-2346.

90. El-Shazly, A.; Abdel-All, M.; Tei, A.; Wink, M. Pyrrolizidine alkaloids from Echium rauwolfii and Echium horridum (Boraginaceae). Z. Naturforsch. 1999, 54c, 295-300.

91. El-Shazly, A.; Sarg, T.; Ateya, A.; Abdel-Aziz, E.; El-Dahmy, S.; Witte, L.; Wink, M. Pyrrolizidine and tetrahydroisoquinoline alkaloids from Echium humile. Phytochemistry 1996, 42, 225-230.

92. Carvalho, J.C.B.; Almeida, H.D.S.; Lobo, J.F.R.; Ferreira, J.L.P.; Oliveira, A.P.; Leandro Rocha, L. Pyrrolizidine alkaloids in two endemic capeverdian Echium species. Biochem. Syst. Ecol. 2013, $50,1-6$. 
93. Colegate, S.M.; Edgar, J.A.; Knill, A.M.; Lee, S.T. Solid-phase extraction and HPLC-MS profiling of pyrrolizidine alkaloids and their N-oxides: A case study of Echium plantagineum. Phytochem. Anal. 2005, 16, 108-119.

94. Culvenor, C.C.J. The alkaloids of Echium plantagineum L. Aust. J. Chem. 1956, 9, 512-520.

95. Roeder, E.; Liu, K.; Bourauel, T. Pyrrolizidine alkaloids from Echium pininana. Phytochemistry 1991, 30, 3107-3110.

96. El-Shazly, A.; Sarg, T.; Ateya, A.; Abdel-Aziz, E.; El-Dahmy, S.; Witte, L.; Wink, M. Pyrrolizidine alkaloids from Echium setosum and Echium vulgare. J. Nat. Prod. 1996, 59, 310-313.

97. Man'ko, I.V. Alkaloids of Cynoglossum officinale and Echium vulgare and standard preparation from Cynoglossum officinale. Farm. Zh. (Kiev) 1964, 19, 22-26.

98. Karimov, A.; Telezhenetskaya, M.V.; Lutfullin, K.; Yunusov, S.Y. Alkaloids of Echium vulgaris and Berberis oblonga. Khim. Prir. Soedin. 1975, 11, 433-434.

99. Dominguez, D.M.; Reina, M.; Santos-Guerra, A.; Santana, O.; Agullo, T.; Lopez-Balbo, C.; Gonzalez-Coloma, A. Pyrrolizidine alkaloids from Canarian endemic plants and their biological effects. Biochem. Syst. Ecol. 2008, 36, 153-166.

100. Suri, O.P.; Jamwal, R.S.; Suri, K.A.; Atal, C.K. Ehretinine, a noval pyrrolizidine alkaloid from Ehretia aspera. Phytochemistry 1980, 19, 1273-1274.

101. L'Empereur, K.M.; Li, Y.; Stermitz, F. Pyrrolizidine alkloids from Hackelia californica and Gnophaela latipennis, and H. californica-hasted arctiid moth. J. Nat. Prod. 1989, 52, 360-366.

102. Li, Y. Two new pyrrolizidine alkaloids from Hackelia californica (Gray) Johnston (Boraginaceae). Huaxue Xuebao 1990, 48, 415-418.

103. Hagglund, K.M.; L'Empereur, K.M.; Roby, M.K.; Stermitz, F.R. Latifoline and latifoline N-oxide from Hackelia floribunda. J. Nat. Prod. 1985, 48, 638-639.

104. Roitman, J.N. Longitubine and neolatifoline, new pyrrolizidine alkaloids from Hackelia longituba. Aust. J. Chem. 1988, 41, 1827-1833.

105. Akramov, S.T.; Shadmanov, Z.; Samatov, A.; Yunusov, S.Y. Alkaloids of Senecio jacobaea, Heliotropium acutiflorum and H. transoxanum. Khim. Prir. Soedin. 1966, 4, 258.

106. Birecka, H.; Frohlich, M.W.; Glickman, M. Free and esterified necines in Heliotropium species from Mexico and Texas. Phytochemistry 1983, 22, 1167-1171.

107. Marquez, V.C. Chromatographic separation of Bulensia retamo, Heliotropium arborescens and Cestrum auriculatum. Bol. Soc. Quim. Peru. 1961, 27, 161-172.

108. Rizk, A.M.; Hammouda, F.M.; Roeder, E.; Wiedenfeld, H.; Ismail, S.I.; Hassan, N.M.; Hosseiny H.A. Occurrence of pyrrolizidine alkaloids in Heliotropium bacciferum Forssk. Sci. Pharm. 1988, 56, 105-110.

109. Farrag, N.M.; Adel-Aziz, E.M.; El-Shafae, A.M.; Ateya, A.M.; El-Domiaty, M.M. Pyrrolizidine alkaloids of Heliotropium bacciferum Frossk from Egypt. Int. J. Pharmacogn. 1996, 34, 374-377.

110. Reina, M.; Mericli, A.H.; Cabbera, R.; Gonzalez-Coloma, A. Pyrrolizidine alkaloid from Heliotropium bovei. Phytochemistry 1995, 38, 355-358.

111. Lakshmanan, A.J.; Shanmugasundaram S. Helibractinecine, a pyrrolizidine alkaloid from Heliotropium bracteatum. Phytochemistry 1994, 36, 245-248. 
112. Lakshmanan, A.J.; Shanmugasundaram, S. Ester alkaloid from Heliotropium bracteatum. Phytochemistry 1995, 40, 291-294.

113. Marquina, G.; Laguna, A.; Velez, H.; Ripperger, H. 9-Angeloylretronecine N-oxide from Heliotropium bursiferum. Pharmazie 1988, 34, 55-56.

114. Eröksüz, H.; Eröksüz, Y.; Ozer, H., Ceribasi, A.O.; Tosun, F.; Tamer, U.; Kizilay, C.A. Toxicity of dietery Heliotropium circinatum to rats. Vet. Hum. Toxicol. 2003, 45, 198-201.

115. Farsam, H.N.; Yassa, N.; Sarkhail, P.; Shafiee, A. New pyrrolizidine alkaloids from Heliotropium crassifolium. Planta Med. 2000, 66, 389-391.

116. Catalfamo, J.L.; Martin, W.B.; Birecka, H. Accumulation of alkaloids and their necines in Heliotropium curassavicum, H. spathulatum and H. indicum. Phytochemistry 1982, 21, 2669-2675.

117. Mohanraj, S.; Subramanian, P.S. Curassavine, an alkaloid from Heliotropium curassavicum Linn. with a $\mathrm{C}_{8}$ necic acid skeleton. J. Chem. Soc. Chem. Comm. 1978, doi: 10.1039/C39780000423.

118. Subramanian, P.S.; Mohanraj, S.; Cockrum, P.A.; Culvenor, C.C.J.; Edgar, J.A.; Farhn J.L.; Smith, L.W. The alkaloids of Heliotropium curassavicum. Aust. J. Chem. 1980, 33, 1357-1363.

119. Davicino, J.G.; Pestchanker, M.J.; Giodano, O.S. Pyrrolizidine alkaloids from Heliotropium curassavicum. Phytochemistry 1988, 27, 960-962.

120. Akramov, S.T.; Kiyamitdinova, F.; Yunusov, S.Y. Alkaloids of Rindera cyclodonata, Rinderia echinata and Heliotropium dasycarpum. Khim. Prir. Soedin. 1967, 3, 288-289.

121. Hammouda, F.M.; Rizk, A.M.; Ismail, S.I.; Atteya, S.Z.; Ghaleb, H.A.; Madkour, M.K.; Pohland, A.E.; Wood, G. Poisonous plants containing edible ones and toxic substances in plant foods. Part 3, Pyrrolizidine alkaloids from Heliotropium digynum Forssk (H. luteum Poir.). Pharmazie 1984, 39, 703-705.

122. Farsam, H.; Yassa, N.; Shafiee, A.; Amanlou, M. Pyrrolizidine alkaloids from Heliotropium disciforme. Pharm. Pharmacol. Lett. 1998, 8, 79-80.

123. Shafiee, A.; Salimi, M.; Farsam, H.; Yassa, N. Pyrrolizidine alkaloids from Heliotropium dissitiflorum Boiss. DARU J. Pharm. Sci. 2002, 10, 168-170.

124. Suri, O.P.; Sawhney, R.S.; Atal, C.K. Pyrrolizidine alkaloids from Heliotropium eichwaldii and Lindelofia spectabilis. Indian J. Chem. 1975, 13, 505-506.

125. Yassa, N.H.; Farsam, H.; Shafiee, A.; Rustaiyan, A. Pyrrolizidine alkaloids from Heliotropium esfandiarii. Planta Med. 1996, 62, 583-584.

126. Yassa, N.H.; Farsam, H.; Rustaiyan, A.; Shafiee, A. Alkaloids of Boraginaceae II [1], pyrrolizidine alkaloids of Heliotropium europaeum L. population Garmsar. J. Sci. Islamic Repub. Iran 1999, 10, 39-42.

127. Tosun, F.; Tamer, U. Determination of pyrrolizidine alkaloids in the seeds of Heliotropium europaeum by GC-MS. J. Fac. Pharm. Ankara 2004, 33, 7-9.

128. Reina, M.; Gonzalez-Coloma, A.; Gutierrez, C.; Cabbera, R.; Henriquez, J.; Villarroel, L. Bioactive saturated pyrrolizidine alkaloid from Heliotropium floridum. Phytochemistry 1997, 46, $845-853$.

129. Constantinidis, T.; Harvala, C.; Skaltsounis, A.L. Pyrrolizidine N-oxide alkaloids of Heliotropium hirsutissimum. Phytochemistry 1993, 32, 1335-1337. 
130. Souza, J.S.N.; Machado, L.L.; Pessoa, O.D.L.; Braz-Filho, R.; Overk, C.R.; Yao, P.; Cordell, G.A.; Lemos, T.L.G. Pyrrolizidine alkaloids from Heliotropium indicum. J. Braz. Chem. Soc. 2005, 16, 1410-1414.

131. Singh, J.P.; Pandey, D.P.; Singh, A.; Singh, R. Alkaloids of Heliotropium indicum. J. Ind. Chem. Soc. 2005, 82, 175-176.

132. Dash, G.K.; Abdullah, M.S. A review on Heliotropium indicum L. (Boraginaceae). Int. J. Pharm. Sci. Res. 2013, 4, 1253-1258.

133. Ravi, S.; Lakshmanan, A.J.; Herz, W. Isolycopsamine, a pyrrolizidine alkaloid from Heliotropium keralense. Phytochemistry 1990, 29, 361-364.

134. Reina, M.; Gonzalez-Coloma, A.; Gutierrez, C.; Cabbera, R.; Henriquez, J.; Villarroel, L. Pyrrolizidine alkaloids from Heliotropium megalanthum. J. Nat. Prod. 1998, 61, 1418-1420.

135. Kiyamitdinova, F.; Akramov, S.T.; Yunusov, S.Y. Alkaloids from the family Boraginaceae. Khim. Prir. Soedin. 1967, 3, 411-412.

136. Mohanraj, S.; Kulanthaivel, P.; Subramanian, P.S.; Herz, W. Helifoline, a pyrrolizidine alkaloid from Heliotropium ovalifolium. Phytochemistry 1981, 20, 1991-1995.

137. Zalkow, L.H.; Gelbaum, L.; Keinan, E. Isolation of the pyrrolizidine alkaloid europine N-oxide from Heliotropium maris-mortui and H. rotundifolium. Phytochemistry 1978, 17, 172.

138. Asibal, C.F.; Gelaum, L.T.; Zalkow, L.H. Pyrrolizidine alkaloids from Heliotropium rotundifolium. J. Nat. Prod. 1989, 52, 726-731.

139. Lakshmanan, A.J.; Shanmugasundaram, S. Heliscabine, a pyrrolizidine ester alkaloid from Heliotropium scabrum. Phytochemistry 1995, 39, 473-475.

140. Roeder, E.; Breitmaier, E.; Birecka, H.; Frohlich, M.W.; Badzies-Crombach, A. Pyrrolizidine alkaloids of Heliotropium spathulatum. Phytochemistry 1991, 30, 1703-1706.

141. Mattocks, A.R. Strigosine, the major alkaloid of Heliotropium strigosum. J. Chem. Soc. 1964, 1974-1977.

142. Singh, B.; Sahu, P.M.; Jain, S.C.; Singh, S. Antineoplastic and antiviral screening of pyrrolizidine alkaloids from Heliotropium subulatum. Pharm. Biol. 2002, 40, 581-586.

143. Crowley, H.C.; Culvenor, C.C.J. The alkaloids of Heliotropium supinum L., with observations on viridifloric acid. Aust. J. Chem. 1959, 12, 694-705.

144. Trigo, J.R.; Witte, L.; Brown, K.S.; Hartmann, T.; Barata, L. pyrrolizidine alkaloids in the acrtiid moth hyalurga syma. J. Chem. Ecol. 1993, 4, 669-679.

145. Medina, J.C.M.; Gauze, G.F.; Vidott, G.J.; Sarragiotto, M.H.; Basso, E.A.; Peixoto, J.L.B. Structural characterization of saturated pyrrolizidine alkaloids from Heliotropium transalpinum var. transalpinum Vell by NMR spectroscopy and theoretical calculation. Tetrahedron Lett. 2009, 50, 2640-2642.

146. Suri, K.A.; Suri, O.P.; Dhar, K L.; Atal, C.K. Chemical composition of Lappula glochidiata and Crotalaria anagyroides. Indian J. Chem. 1978, 16B, 78.

147. Man'ko, I.V.; Vasil'kov, P.N. Lappula intermedia alkaloids. Tr. Leninger. Khim.-Farm. Inst. 1968, 26, 166.

148. Wiedenfeld, H.; Amarsanaa, B.; Altanchimeg, D.; Narantuya, S. Pyrrolizidine alkaloid containing plants in Mongolian traditional medicine: Lappula myosotis Moench. Sci. Pharm. 2005, 73, 139-145. 
149. Kelly, H.A.; Robins, D.J. Pyrrolizidine alkaloids from Lindelofia longiflora. Fitoterapia 1990, 61, 89-90.

150. Akramov, S.T.; Kiyamitdinova, F.; Yunusov, S.Y. Alkaloids of Solenanthus turkestanicus, Lindelofia olgae, and Trachelanthus korolkovii. Dokl. Akad. Nauk Uzb. SSR 1962, 19, 29.

151. Akramov, S.T.; Kiyamitdinova, F.; Yunusov, S.Y. Alkaloids of Solenanthus circinatus, Paracaryum himalayense and Lindelofia pterocarpa. Dokl. Akad. Nauk Uzb. SSR 1964, 21, 28.

152. Akramov, S.T.; Kiyamitdinova, F.; Yunusov, S.Y. Study of Rindera and Lindelofia. Dokl. Akad. Nauk Uzb. SSR 1965, 22, 35-38.

153. Pietrosiuk, A.; Syklowska-Baranek, K.; Wiedenfeld, H.; Wolinowska, R.; Furmanowa, M.; Jaroszyk, E. The shikonin derivatives and pyrrolizidine alkaloids in hairy root cultures of Lithospermum canescens (Michx.) Lehm. Plant Cell Rep. 2006, 25, 1052-1058.

154. Wiedenfeld, H.; Pietrosiuk, A.; Furmanowa, M.; Roeder, E. Pyrrolizidine alkaloids from Lithospermum canescens Lehm. Z. Naturforsch. 2003, 58c, 173-176.

155. Roeder, E.; Renge, B. Pyrrolizidine alkaloids from Lithospermum erythrorhizon. Phytochemistry 1990, 29, 690-693.

156. Krenn, L.; Wiedenfeld, H.; Roeder, E. Pyrrolizidine alkaloids from Lithospermum officinale. Phytochemistry 1994, 37, 275-277.

157. Li, Y.; Stermitz, F.R. Pyrrolizidine alkaloids from Mertensia species of Colorado. J. Nat. Prod. 1988, 51, 1289-1290.

158. Ogihara, K.; Miyagi, Y.; Higa, M.; Yogi, S. Pyrrolizidine alkaloids from Messerschmidia argentea. Phytochemistry 1997, 44, 545-547.

159. Resch, J.F.; Rosberger, D.F.; Meinwald, J.; Appling, J.W. Biologically active pyrrolizidine alkaloids from the true forget-me-not, Myosotis scorpioides. J. Nat. Prod. 1982, 45, 358-362.

160. Roeder, E.; Bourauel, T. Pyrrolizidine alkaloids from Neatostema apulum. Phytochemistry 1992, 31, 3613-3615.

161. Roeder, E.; Wiedenfeld, H.; Kroeger, R.; Teppner, H. Pyrrolizidine alkaloids of three taxa of Onosma (Boraginaceae-Lithospermeae). Phyton 1993, 33, 41-49.

162. Roeder, E.; Wiedenfeld, H.; Kersten, R.; Kröger, R. Determination of open chain pyrrolizidine alkaloids by capillary gas chromatography. Planta Med. 1990, 56, 522.

163. El-Shazly, A.; Adel-Ghani, A.; Wink, M. Pyrrolizidine Alkaloids from Onosma arenaria Waldst. and Kit (Boraginaceae). Biochem. Syst. Ecol. 2003, 31, 477-485.

164. Damianakos, H.; Sotiroudis, G.; Chinou, I. Pyrrolizidine alkaloids from Onosma erecta. J. Nat. Prod. 2013, 76, 1892-1835.

165. Mellidis, A.S.; Papageorgiou, V.P. Pyrrolizidine alkaloids of the plant Onosma heterophylla. Chem. Chron. 1988, 17, 67-73.

166. Kretsi, O.; Aligiannis, N.; Skaltsounis, A.L.; Chinou, I.B. Pyrrolizidine alkaloids from Onosma leptantha. Helv.Chim. Acta 2003, 86, 3136-3140.

167. Mroczek, T.; Ndjoko, K.; Glowniak, K.; Hostetmann, K. On-line structure characterization of pyrrolizidine alkaloids in Onosma stellulatum and Emilia coccinea by liquid chromatography-ion-trap mass spectrometry. J. Chromatogr. A. 2004, 1056, 91-97.

168. Haberer, W.; Witte, L.; Hartmann, T.; Dobler, S. Pyrrolizidine alkaloids in Pulmonaria obscura. Planta Med. 2002, 68, 480-482. 
169. Mandić, B.M.; Simić, M.R.; Vučković, I.M.; Vujisić, L.V.; Novaković, M.M.; Snežana, S.; Trifunović, S.S.; Snežana, D.; Nikolić-Mandić, S.D.; Vele, V.; Tešević, V.V.; Vajs, V.V.; Milosavljević, S.M. Pyrrolizidine alkaloids and fatty acids from the endemic plant species Rindera umbellata and the effect of lindelofine- $N$-oxide on tubulin polymerization. Molecules 2013, 18, 10694-10706.

170. Kurucu, S.; Kartal, M.; Choudary, M.I.; Topcu, G. Pyrrolizidine Alkaloids from Symphytum sylvaticum Boiss. subsp. sepulcrale. (Boiss. \& Bal.) Greuter \& Burdet var. sepulcrale and Symphytum aintabicum Hub.-Mor. \& Wickens. Turk J Chem. 2002, 26, 195-199.

171. Huizing, H.J.; Gadella, T.W.G.; Kliphuis, E. Chemotaxonomical investigation of the Symphytum officinale polyploidy complex and S. asperum (Boraginaceae): The pyrrolizidine alkaloids. Plant Syst. Evol. 1982, 140, 279-292.

172. Roeder, E.; Bourauel, T.; Neuberger, V. Symviridine, a new pyrrolizidine alkaloids from Symphytum species. Phytochemistry 1992, 31, 4041-4042.

173. Jaarsma, T.A.; Lohmanns, E.; Gadella, T.W.J.; Malingre, T.M. Chemotaxonomy of the Symphytum officinale agg. (Boraginaceae). Plant Syst. Evol. 1989, 167, 113-127.

174. Mel'kumova, Z.V.; Telzhenetskaya, M.V.; Yunusov, S.Y.; Man'ko, I.V. Refinement of the structure of asperumine. Khim. Prir. Soed. 1974, 4, 478-480.

175. Huizing, H.J.; Malinerg, T.M. Purification and separation of pyrrolizidine alkaloids from Boraginaceae on a polystyrene-divinylbenzene resin. J. Chromatogr. 1979, 176, 274-279.

176. Jaarsma, T.A.; Lohmanns, E.; Hendriks, H.; Gadella, T.W.J.; Malingre, T.M. Chemo-and karyotaxonomic studies on some rhizomatous species of the genus Symphytum (Boraginaceae). Plant Syst. Evol. 1990, 169, 31-39.

177. Liu, F.; Wan, S.Y.; Jiang, Z.; Li, S.F.; Ong, E.S.; Osorio, J.C. Determination of pyrrolizidine alkaloids in comfrey by liquid chromatography-electrospray ionisation mass spectrometry. Talanta 2009, 15, 916-923.

178. Furuya, T.; Araki, K. Studies on constituents of crude drugs. 1. Alkaloids of Symphytum officinale Linn. Chem. Pharm. Bull. 1968, 16, 2512-2516.

179. Roeder, E.; Wiedenfeld, H.; Stengl, P. 13C-NMR Daten der stereoisomeren Alkaloide aus Symphytum officinale L. Arc. Pharm. (Weinheim) 1982, 315, 87-88.

180. Culvenor, C.C.J.; Edgar, J.A.; Frahn, J.L.; Smith, L.W. The alkaloids of Symphytum $\times$ uplandicum (Russian comfrey). Aust. J. Chem. 1980, 33, 1105-1113.

181. Omar, M.; Defeo, J.; Youngken, H.W. Chemical and toxicity studies of Trichodesma africanum L. J. Nat. Prod. 1983, 46, 153-156.

182. O'Kelly, J.; Sargeant, K. Supinine from the seeds of Trichodesma zeylanicum R. Br. J. Chem. Soc. 1961, 484.

183. Khassanova, M.A.; Abdullaev, U.A.; Telezhenetskaya, M.V.; Yunusov, S.Yu. Structure of uluganine. Khim. Prir. Soedin. 1974, 6, 809-810.

184. Roeder, E.; Wiedenfeld, H.; Schraut, R. Pyrrolizidine alkaloids from Alkanna tinctoria. Phytochemistry 1984, 23, 2125-2126.

185. Culvenor, C.C.J.; Smith, L.W. Aquaternary N-dihydropyrrolizinomethyl derivative of heliotrine from Heliotropium Europaeum. Tetrahedron Lett. 1969, 41, 3603-3606. 
186. Culvenor, C.C.J.; Johns, S.R.; Smith, L.W. Acetyllasiocarpine, an alkaloids of Heliotropium europaeum. Aust. J. Chem. 1975, 28, 2319-2322.

187. Ravi, S.; Ravikumar, R.; Lakshmanan, A.J. Pyrrolizidine alkaloids from Cynoglossum furcatum. Nig. J. Nat. Prod. Med. 2007, 11, 87-89.

188. Crews, C.; Berthiller, F.; Krska, R. Update on analytical methods for toxic pyrrolizidine alkaloids. Anal. Bioanal. Chem. 2010, 396, 327-338.

189. Crews, C. Methods for Analysis of Pyrrolizidine Alkaloids in Natural Products; Ramawat, K.G., Merillon, J.M., Eds.; Springer-Verlag: Berlin \& Heidelberg, Germany, 2013; pp. 1049-1068.

190. Zalkow, L.H.; Asibal, C.F.; Glinski, J.A.; Bonetti, S.J.; Gelbaum, L.T.; van Derveer, D.; Powis, G. Macrocyclic pyrrolizidine alkaloids from Senecio anonymus. Separation of a complex alkaloid extract using droplet counter-current chromatography. J. Nat. Prod. 1988, 51, 690-702.

191. Bicchi, C.; Caniato, R.; Tabacchi, R.; Tsoupras, G. Capillary gas chromatography/positive and negative ion chemical ionization mass spectrometry on pyrrolizidine alkaloids of Senecio inaequidens using ammonia and hydroxyl ions as the reagent species. J. Nat. Prod. 1989, 52, 32-41.

192. Stelljes, M.E.; Kelley, R.B.; Molyneux, R.J.; Seiber, J.N. GC-MS determination of pyrrolizidine alkaloids in four Senecio species. J. Nat. Prod. 1991, 54, 759-773.

193. Witte, L.; Rubiolo, P.; Bicchi, C.; Hartmann, T. Comparative analysis of pyrrolizidine alkaloids from natural sources by gas chromatography-mass spectrometry. Phytochemistry 1993, 32, 187-196.

194. El-Shazly, A. Pyrrolizidine alkaloid profiles of some Senecio species from Egypt. Z. Naturforsch. 2002, 57c, 429-433.

195. Asres, K.; Sporer, F.; Wink, M. Patterns of pyrrolizidine alkaloids in 12 Ethiopian Crotalaria species. Biochem. Syst. Ecol. 2004, 32, 915-930.

196. Crews, C.; Startin, J.R.; Clarke, P.A. Determination of pyrrolizidine alkaloids in honey from selected sites by solid phase extraction and HPLC-MS. Food Addit. Contam. 1997, 14, 419-428.

197. Lin, G.; Zhou, K.Y.; Zhao, X.G.; Wang, Z.T.; But, P.P. Determination of hepatotoxic pyrrolizidine alkaloids by on-line high performance liquid chromatography mass spectrometry with an electrospray interface. Rapid Commun. Mass Spectrom. 1998, 12, 1445-1456.

198. Zhang, F.; Wang, C.H.; Wang, W.; Chen, L.X.; Ma, H.Y.; Zhang, C.F.; Zhang, M.; Bligh, S.W.; Wang, Z.T. Quantitative analysis by HPLC-MS2 of the pyrrolizidine alkaloid adonifoline in Senecio scandens. Phytochem. Anal. 2008, 19, 25-31.

199. Ruan, J.; Li, N.; Xia, Q.; Fu, P.P.; Peng, S.; Ye, Y.; Lin, G. Characteristic ion clusters as determinants for the identification of pyrrolizidine alkaloid N-oxides in pyrrolizidine alkaloid-containing natural products using HPLC-MS analysis. J. Mass Spectrom. 2012, 47, 331-337.

200. Neuner-Jehle, N.; Nesvadba, H.; Spiteller, G. Anwendung der Massenspektrometric zur Strukturaufklärung von Alkaloiden, 6. Mitt. Mh. Chem. 1965, 96, 321-338. (In German)

201. Pedersen, E.; Larsen, E. Mass spectrometry of some pyrrolizidine alkaloids. Org. Mass Spectrom 1970, 4, 249-256.

202. Culvenor, C.C.J.; Edgar, J.A.; Frahn, J.L.; Smith, L.W.; Ulubelen, A.; Doganca, S. The structure of anadaloine. Aust. J. Chem. 1975, 28, 173-178.

203. Wuilloud, J.C.A.; Gratz, S.R.; Gamble, B.M.; Wolnik, K.A. Simultaneous analysis of hepatotoxic pyrrolizidine alkaloids and $\mathrm{N}$-oxides in comfrey root by LC-ion trap mass spectrometry. Analyst 2004, 129, 150-156. 
204. Roeder, E.; Wiedenfeld, H.; Hoeing, A. Pyrrolizidinalkaloide aus Senecio aureus. Planta Med. 1983, 49, 57-59.

205. Flores, A.S.; de Azevedo Tozzi, A.M.G.; Trigo, J.R. Pyrrolizidine alkaloid profiles in Crotalaria species from Brazil: Chemotaxonomic significance. Biochem. Syst. Ecol. 2009, 37, 459-469.

206. Mead, E.W.; Looker, M.; Gardner, D.R.; Stermitz, F.R. Pyrrolizidine alkaloids of Liatris punctata and its root parasite, Castilleja integra. Phytochemistry 1992, 31, 3255-3257.

207. Tei, A. Identifizierung und Strukturaufklärung von Alkaloiden und anderen Sekundärstoffen mit Hilfe von GC-MS und Kernresonanzspektroskopie. Ph.D. Thesis, Ruprecht-Karls-Universität Heidelberg, Heidelberg, Germany, 2000.

208. Stermiz, F.R.; L'Empereur, K.M. Identity of "subulasine N-oxide" with $1 \beta, 2 \beta$-epoxy-1 $\alpha$ -

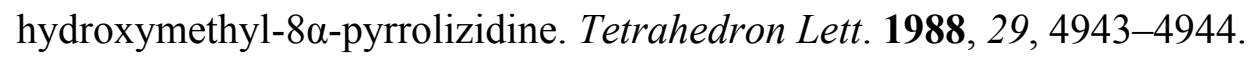

209. Griffin, C.T.; Danaher, M.; Elliott, C.T.; Kennedy, D.G.; Furey, A. Detection of pyrrolizidine alkaloids in commercial honey using liquid chromatography-ion trap mass spectrometry. Food Chem. 2013, 136, 1577-1583.

210. Martinello, M.; Cristofoli, C.; Gallina, A.; Mutinelli, F. Easy and rapid method for the quantitative determination of pyrrolizidine alkaloids in honey by ultra performance liquid chromatography-mass spectrometry: An evaluation in commercial honey. Food Control 2014, 37, $146-152$.

211. Logie, C.G.; Grue, M.R.; Liddell, J.R. Proton NMR spectroscopy of pyrrolizidine alkaloids. Phytochemistry 1994, 37, 43-109.

212. Roeder, E. Carbon-13 NMR spectroscopy of pyrrolizidine alkaloids. Phytochemistry 1990, 29, $11-29$.

213. Bober, M.A.; Milco, L.A.; Miller, R.B.; Mount, M.; Wicks, B.; Kurth, M.J. A competitive enzyme-linked immunosorbent assay (ELISA), to detect retronecine and monocrotaline in vitro. Toxicon. 2004, 27, 1059-1064.

214. Roeder, E.; Pflueger, T. Analysis of pyrrolizidine alkaloids: a competitive enzyme-linked immunoassay (ELISA) for the quantitative determination of some toxic pyrrolizidine alkaloids. Nat. Toxins. 1995, 3, 305-309.

215. Lee, S.T.; Schoch, T.K.; Stegelmeier, B.L.; Gardner, D.R.; Than, K.A.; Molyneus, R.J. Development of enzyme-linked immunosorbent assays for the hepatotoxic riddelliine and reddelliine $\mathrm{N}$-oxide. J. Agric. Food Chem. 2001, 49, 4144-4151.

216. Cavallaro, V.; Than, K.A.; Colegate, S.M.; Edgar, J.A. An indirect competitive ELISA for pyrrolizidine alkaloids of Heliotropium europaeum. In Poisonous Plants and Related Toxins; Acamovic, T., Stewart, C.S., Pennycott, T.W., Eds; CABI publishing: Wallingford, UK, 2004; pp. 114-119.

217. Lee, S.T.; Knill, A.; Michalewicz, A.; Stevens, V.; Colegate, S.M. Heliotropium europaeum alkaloids: A quaternary pyrrolizidine alkaloids approach to elisa development. In Poisonous Plant Global Research and Solution; United States Department of Agriculture: Washington, DC, USA, 2007; Chapter 81, pp. 476-480.

218. Von Borstel, K.; Hartmann, T. Selective uptake of pyrrolizidine N-oxides by cell suspension cultures from pyrrolizidine alkaloid producing plants. Plant Cell Rep. 1986, 5, 39-42. 
219. Van Dam, N.M.; Verpoorte, R.; Meijden, E.V.D. Extreme differences in pyrrolizidine alkaloid levels between leaves of Cynoglossum officinale. Phytochemistry 1994, 37, 1013-1016.

220. Frölich, C.; Ober, D.; Hartmann, T. Tissue distribution, core biosynthesis and diversification of pyrrolizidine alkaloids of the lycopsamine type in three Boraginaceae species. Phytochemistry 2007, 68, 1026-1037.

221. Abd El-Mawla, A.M. Effect of cretin elicitors on production of pyrrolizidine alkaloids in hairy root cultures of Echium rauwolfii. Pharmazie 2010, 65, 224-226.

222. Marin-Loaiza, J.C.; Ernst, L.; Beuerle, T.; THeuring, C.; Cespedes, C.L.; Hartmann, T. Pyrrolizidine alkaloids of the endemic Mexican genus Pittocaulon and assignment of stereoisomeric 1,2-saturated necine bases. Phytochemistry 2008, 69, 154-167.

223. Okusa, P.N.; Penge, O.; Devleeschouwer, M.; Duez, P. Direct and indirect antimicrobial and antioxidant activity of Cordia dilletii De Wild (Boraginaceae). J. Ethanopharmacol. 2007, 112, 476-481.

224. Joosten, L.; van Veen, J.A. Defensive properties of pyrrolizidine alkaloids against microorganisms. Phytochem. Rev. 2011, 10, 127-136.

225. Wink, M. Allelochemical properties and raison d'être of alkaloids. In The Alkaloids; Cordell, G., Ed.; Academic Press: New York, NY, USA, 1993; vol. 43, pp. 1-118.

226. Boppre, M. Insects pharmacophagously utilizing defensive plant chemicals (pyrrolizidine alkaloids). Naturwissenschaften 1986, 73, 17-26.

227. Boppre, M. Lepidoptera and pyrrolizidine alkaloids. Exemplification of complexity in chemical ecology. J. Chem. Ecol. 1990, 16, 165-185.

228. Von Nickisch-Rosenegk, E.; Wink, M. Sequestration of pyrrolizidine alkaloids in several arctiid moths (Lepidoptera: Arctiidae). J. Chem. Ecol. 1993, 19, 1889-1903.

229. Dobler, S.; Haberer, W.; Witte, L.; Hartmann, T. Selective sequestration of pyrrolizidine alkaloids from diverse host plants by Longitarsus flea beetles. J. Chem. Ecol. 2000, 5, 1281-1298.

230. Honda, Y.; Honda, K.; Omura, H. Major components in the hairpencil secretion of a butterfly, Euploea mulciber (Lepidoptera, Danaidae): Their origin and male behavioral responses to pyrrolizidine alkaloids. J. Insect Physiol. 2006, 52, 1043-1053.

231. Beuerle, T.; Theuring, C.; Klewer, N.; Schulz, S.; Hartmann, T. Absolute configuration of the creatonotines and callimorphines, two classes of arctiid-specific pyrrolizidine alkaloids. Insect Biochem. Mol. Biol. 2007, 37, 80-89.

232. Macel, M. Attract and deter: A dual role for pyrrolizidine alkaloids in plant-insect interaction. Phytochem. Rev. 2011, 10, 75-82.

233. Denmark, S.E.; Hurd, A.R. Synthesis of (+)-casuarine. J. Org. Chem. 2000, 65, 2875-2886.

234. Asano, N.; Ikeda, K.; Kasahara, M.; Arai, Y.; Kizu, H. Glycosidase-inhibiting pyrrolidines and pyrrolizidines with long side chain in Scilla peruviana. J. Nat. Prod. 2004, 67, 846-850.

235. Garcia-Moreno, M.I.; Rodriguez-Lucena, D.; Mellet, C.O.; Garcia Fernandez, J.M. Pseudoamide-type pyrrolidine and pyrrolizidine glycomimetics and their inhibitory activities against glycosidases. J. Org. Chem. 2004, 69, 3578-3581.

236. Nibret, E.; Sporer, F.; Asres, K.; Wink, M. Antitrypanosomal and cytotoxic activity of pyrrolizidine alkaloid-producing plants of Ethiopia. J. Pharm. Pharmacol. 2009, 61, 801-808. 
237. Singh, B.; Sahu, P.M.; Singh, S. Antimicrobial activity of alkaloids from Heliotropium subulatum. Fitoterapia 2002, 73, 153-155.

238. Wink, M. Evolution of secondary metabolites in legumes (Fabaceae). S. Afr. J. Bot. 2013, 89, $164-175$.

239. Pfister, J.A.; Molyneux, R.J.; Baker, D.C. Pyrrolizidine alkaloid content of hounds tongue (Cynoglossum officinale L.). J. Range Managem. 1992, 45, 254-256.

(C) 2014 by the authors; licensee MDPI, Basel, Switzerland. This article is an open access article distributed under the terms and conditions of the Creative Commons Attribution license (http://creativecommons.org/licenses/by/3.0/). 